September 25, 2018

\title{
The Exotic Barium Bismuthates
}

\author{
A. Taraphder* \\ Laboratoire d'etudes des Proprietes Electroniques des Solides, \\ Centre National de la Recherche Scientifique, B.P. 166, 38042 Grenoble, France, \\ and \\ Rahul Pandit ${ }^{\dagger}$, H. R. Krishnamurthy ${ }^{\dagger}$, and T.V. Ramakrishnan \\ Department of Physics, Indian Institute of Science, Bangalore - 560012, India
}

\begin{abstract}
We review the remarkable properties, including superconductivity, charge-densitywave ordering, and metal-insulator transitions, of lead- and potassium-doped barium bismuthate. We discuss some of the early theoretical studies of these systems. Our recent theoretical work, on the negative- $U$, extended-Hubbard model for these systems, is also described. Both the large- and intermediate- $U$ regimes of this model are examined, using mean-field and random-phase approximations, particularly with a view to fitting various experimental properties of these bismuthates. On the basis of our studies, we point out possibilities for exotic physics in these systems. We also emphasize the different consequences of electronic and phonon-mediated mechanisms for the negative $U$. We show that, for an electronic mechanism, the semiconducting phases of these bismuthates must be unique, with their transport properties dominated by charge $\pm 2 e$ Cooperon bound states. This can explain the observed difference between the optical and transport gaps. We propose other experimental tests for this novel mechanism of charge transport and comment on the effects of disorder.

PACS numbers: $74.10+\mathrm{v}, 74.65+\mathrm{n}$
\end{abstract}

*Present address: Mehta Research Institute of Mathematics and Mathematical Physics, 10 Kasturba Gandhi Marg, Allahabad-211002, India.

${ }^{\dagger}$ also at Jawaharlal Nehru Centre for Advanced Scientific Research, Bangalore 560012, India. 
The materials $\mathrm{BaPb}_{1-x} \mathrm{Bi}_{x} \mathrm{O}_{3}$, and $\mathrm{Ba}_{1-x} \mathrm{~K}_{x} \mathrm{BiO}_{3}$, derived from barium bismuthate, $\mathrm{BaBiO}_{3}$, have many remarkable properties [1, 2, 3, 4, 5, 6, 7, 8, 9, 10. These include structural and metal-semiconductor transitions as $x$ and the temperature $T$ are varied; diamagnetic, semiconducting (CDW ordered?) phases even in the presence of a partially filled $\mathrm{Bi} 6 \mathrm{~s}-\mathrm{O} 2 \mathrm{p}_{\sigma}$ antibonding band, with optical and transport gaps $(2 \mathrm{eV}$ and $0.24 \mathrm{eV}$, respectively, at $x=0$ ) differing by nearly an order of magnitude; and superconducting $T_{c}$ 's (13K and $34 \mathrm{~K}$, respectively, for $\mathrm{Pb}$ and $\mathrm{K}$ doping) that are $3-5$ times higher than for other three-dimensional oxide systems with similar, low densities of states at the Fermi level. However, partly because of all the attention devoted to the high- $T_{c}$ cuprates, these bismuthates have not been studied extensively, either experimentally or theoretically.

We review here the experimental properties of lead- and potassium-doped barium bismuthate, give a summary of some early theoretical studies of these systems, and then provide an overview of our recent theoretical work [9, 10], which uses the negative- $U$, extended-Hubbard model for these systems. The principal goal of our review is to consolidate what is known (experimentally and theoretically) about these remarkable materials. We have organised the review as follows: Section I is devoted to the phenomenology of lead- and potassium-doped barium bismuthate. Section II is devoted to a survey of the models that have been put forth for these systems and to some of the early theoretical work on them. Sections III and IV are devoted to our work on these systems based on the negative- $U$, extended-Hubbard model: Both the large- $U$ (Sec.III) and intermediate- $U$ (Sec.IV) regimes of this model are examined, using mean-field and random-phase approximations, particularly with a view to fitting various experimental properties of these bismuthates. Section IV ends with some concluding remarks in which we point out, on the basis of our studies, possibilities for exotic physics in these systems, the different consequences of electronic and phonon-mediated mechanisms for the negative $U$, and new experimental tests for them. 


\section{Phenomenology of Barium Bismuthates}

This Section contains a detailed account of the normal and superconducting properties of the barium bismuthates. We begin with an introduction (Sec.I.1), summarize the remarkable experimental properties of these systems (Sec.I.2), and then discuss various experimental observations and their interpretations (Sec.I.3). In Sec.I.4 we give a short account of the electronic structure and configuration of these systems as obtained from band-structure calculations.

\section{I.1 Introduction}

Compounds containing elements of Group VA (e.g., Sb, Bi, etc.) have drawn considerable attention [12] from chemists for a long time. According to their electronic configurations $\left(n s^{2} p^{3}\right.$ in their outer shell $n$; e.g., for $\mathrm{Bi} n$ is 6 and the electronic configuration is [Xe $4 f^{14} 5 d^{10} 6 s^{2} 6 p^{3}$, where [Xe] corresponds to filled shells of xenon) they should form compounds with oxidation states $3^{+}$or $5^{+}$, as these would yield closed, outer-shell configurations for the corresponding cations. Indeed, these elements generally form compounds with valences $3^{+}$or $5^{+}$, skipping the intermediate valence $4^{+}$. Thus chemists were intrigued when they were able to prepare compounds in which these cations have a nominal valence $4^{+}$. However, the actual valences of these cations (in these compounds) turned out to be controversial; e.g., the valence of $\mathrm{Sb}$ in the halides of the type $\mathrm{A}_{2}^{1+} \mathrm{SbX}_{6}^{1-},(\mathrm{X}$ represents a halogen and $\mathrm{A}$ a monovalent cation like $\mathrm{NH}_{4}$ ): Originally these compounds were regarded as instances of tetravalent Sb. Finally, careful Sb-Mössbauer studies [12] confirmed that the actual compounds are like $\mathrm{A}_{4}^{1+} \mathrm{Sb}^{3+} \mathrm{Sb}^{5+} \mathrm{X}_{12}^{1-}$, i.e., the $\mathrm{Sb}^{4+}$ ions charge disproportionate into $\mathrm{Sb}^{3+}$ and $\mathrm{Sb}^{5+}$ ions.

A similar controversy has existed regarding the valence of $\mathrm{Bi}$ in $\mathrm{BaBiO}_{3}$, which was thought to be an example of a compound with tetravalent $\mathrm{Bi}$. Here also there is some 
experimental evidence (see later) that $\mathrm{Bi}$ charge disproportionates into $\mathrm{Bi}^{3+}$ and $\mathrm{Bi}^{5+}$ configurations, thus skipping the intermediate valence $4^{+}$. (Since Bi is Mössbauer inactive, a direct verification, as in the case of Sb Halides, is not possible.) The renewed and vigorous interest in this system was motivated by the discovery [1] that these systems show superconductivity when doped with lead, with a maximum transition temperature $T_{c}$ of $13 \mathrm{~K}$. For a long time thereafter, this was the highest superconducting $T_{c}$ observed amongst compounds containing no transition metal. More recently, it has been reported [3, $4,5,13,14,15]$ that, on doping with potassium (which replaces Ba), this system shows an even higher superconducting transition temperature $\left(T_{c} \simeq 30 \mathrm{~K}\right.$ at $40 \% \mathrm{~K}$ doping$)$.

There are, in fact, about fifteen elements (not restricted to Group VA) in the periodic table that show this extraordinary property of valence skipping [12, 18]. For example, Ga, In and $\mathrm{Tl}$ show valences of $1^{+}$and $3^{+}, \mathrm{Ge}, \mathrm{Sn}$ and $\mathrm{Pb}$ of $2^{+}$and $4^{+}$and $\mathrm{Bi}$ and $\mathrm{Sb}$ of $3^{+}$and $5^{+}$. $\mathrm{V}$ and $\mathrm{Nb}$ form compounds with valence states $3^{+}$and $5^{+}$, Ti with $2^{+}$and $4^{+}$. In all these cases the intermediate valence states are either unstable or metastable. The formal valence state of these cations determines, to a large extent, the shape and orientation of the other orbitals (principally of the anion) in the compound they form; hence any theoretical calculations and predictions of the chemical nature and physical properties of these compounds would necessarily require this information. In addition, the microscopic

physics underlying valence skipping is extremely important for the construction of suitable theoretical models.

\section{I.2 Summary of Important Experimental Observations}

The compounds $\mathrm{BaPb}_{1-x} \mathrm{Bi}_{x} \mathrm{O}_{3}$ and $\mathrm{Ba}_{1-x} \mathrm{~K}_{x} \mathrm{BiO}_{3}$ and those in which $\mathrm{Pb}$ is replaced by $\mathrm{Sb}$ [四, Ba by $\mathrm{Sr}$, and $\mathrm{K}$ by $\mathrm{Rb}$ [3, 4, 5, 13, 14, 15] have many interesting properties [2, 6, 17, 16, 17, 19, 20, 21]. 
(1) $\mathrm{BaBiO}_{3}$ is an insulator even though it has a half-filled $\mathrm{Bi} 6 \mathrm{~s}-\mathrm{O} 2 \mathrm{p}_{\sigma}$ band.

(2) The alloys exhibit a metal-semiconductor transition as the concentration of the

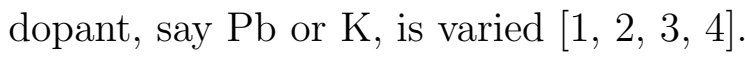

(3) However, at all dopant concentrations these systems are diamagnetic [2, 21, 22, 23, 24], so their semiconducting phases are clearly not Mott insulators.

(4) In their semiconducting phases these alloys exhibit two energy gaps differing by nearly an order of magnitude $\left(2 \mathrm{eV}\right.$ and $0.24 \mathrm{eV}$ in $\left.\mathrm{BaBiO}_{3}\right)$; the larger gap shows up clearly in measurements of the optical conductivity and the smaller one in the temperature dependence of the resistivity [2, 9, 25, 47]. The transport gap seems to be inaccessible to optical-reflectance, photoconductivity, or photoacoustic spectroscopy.

(5) The semiconducting phases of the lead-doped alloys show many properties (e.g., two Bi-O distances) that indicate the presence of charge-density-wave (CDW) ordering [2, 6, 0, 8, 19, 26, 27, 28].

(6) These alloys undergo various structural transitions as functions of the concentration $x$ and the temperature $T$; the structures exhibited by these alloys - ranging from monoclinic to cubic - can be obtained by slight distortions of an underlying cubic perovskite structure [8, 26, 29].

(7) Both the lead- and potassium-doped systems are type-II superconductors with $T_{c}$ 's that are $3-5$ times higher than those for other three-dimensional oxide systems with similar densities of states at the Fermi level (calculated from experimentally obtained values of $\gamma$, the Sommerfeld parameter [2, 6, 7, 30, 31, 32]).

Thus these systems are quite unusual and a theoretical understanding of their properties is a challenging task, further complicated by the limited amount of experimental 
data available and by the lack of agreement among experimentalists on many of the observations and their interpretations.

Recently 33] a family of Bi-based layered superconducting compounds have been synthesized which are isostructural with the tetragonal cuprate superconductor $\mathrm{La}_{2} \mathrm{CuO}_{4}$. These compounds, $\mathrm{Ba}_{2-x} \mathrm{~K}_{x} \mathrm{~Pb}_{1-y} \mathrm{Bi}_{y} \mathrm{O}_{4-y}$, show an insulator to metal transition as a function of potassium doping. The metallic phase shows superconductivity with a highest transition temperature of about $14 \mathrm{~K}$. We will not discuss these compounds in this review.

\section{I.3 Experimental Overview}

This Section contains an overview of the experimental data available for these bismuthates. We have tried, as far as possible, to collect results which are backed by more than one group and which have been obtained by using single-crystal samples.

\section{I.3.1 Phase Diagram}

The structures of barium bismuthates can all be obtained from slight distortions of an underlying cubic perovskite structure shown in Fig.I.1(a). In this structure, $\mathrm{Bi}$ (or $\mathrm{Pb}$ ) atoms occupy the corners of a cubic unit cell, $\mathrm{O}$ atoms the face centres, and $\mathrm{Ba}$ (or K) atoms the body centres. There are octahedral clusters of $\mathrm{O}$ atoms around each $\mathrm{Bi}$ (or $\mathrm{Pb}$ ) atom. Slight rotations or breathing-mode distortions of these octahedra lead to a variety of noncubic structures. For example, the distortions that yield the pure $\mathrm{BaBiO}_{3}$ structure are shown in Fig.I.1b. Here there are two distinct nearest-neighbour Bi-O distances $2.28 \stackrel{\circ}{A}$ and $2.12 \AA$ : oxygen octahedra around one of the sublattices of the underlying cubic structure contract and those around the other sublattice expand. This can be interpreted in terms of a freezing of the breathing mode of the oxygen octahedra leading to a CDW: Tilts of the oxygen octahedra further lower the symmetry of $\mathrm{BaBiO}_{3}$. It is 
not surprising, therefore, that both $\mathrm{BaPb}_{1-x} \mathrm{Bi}_{x} \mathrm{O}_{3}$ and $\mathrm{Ba}_{1-x} \mathrm{~K}_{x} \mathrm{BiO}_{3}$ show rich phase diagrams with many structural transitions. Their phase diagrams in the temperature $T$ and the concentration $x$ plane are shown schematically in Figs. I.2a and I.2b, respectively 8, 19, 29.

In addition to these transitions both these bismuthates show metallic, superconducting, and semiconducting phases (Figs. I.2a and I.2b), the transitions between which show up most clearly in transport properties. The orders of these transitions are not very clear: The metal-superconductor transition is most probably second order. Some workers have suggested that there is a first-order transition at finite temperature from the metallic phase to the semiconducting phase [19] (hence the hatched, two-phase region in the phase diagram of Fig.I.2a). From the available experimental data it is not clear whether the superconducting and semiconducting phases coexist along any phase boundary and, if so, what the order of the transition along this boundary is [2, 9]. However, the maximum value of the superconducting $T_{c}$ seems to occur at nearly the same value of $x$ as the metal-semiconductor transition.

Electron diffraction study of the structural transitions in $\mathrm{BaPb}_{1-x} \mathrm{Bi}_{x} \mathrm{O}_{3}$ has been performed on powder samples by Koyama and Ishimara [16] and by Minami [17 recently. The phase diagram as a function of doping $x$ that Koyama and Isimara get differs from the one reported by Cox and Sleight [26] earlier. These authors do not get any orthorhombic phase in their electron diffrcation studies. They find evidence for tetragonal and monoclinic phases at low temperature and a high temperature $(\simeq 500 \mathrm{~K})$ cubic phase (the transition occuring at $x=0.4$ ). They have also investigated the existence of any incommensurate phase and concluded in the negative, in agreement with Cox and Sleight.

In early studies of the lead-doped compound [1] it was suggested that superconductivity could only occur in the tetragonal phase; however, subsequent work seems to suggest that it can also be obtained in specimens with orthorhombic symmetry [2]. In the 
potassium-doped compound, superconductivity has been observed only in the cubic phase [29]. In both the potassium- and lead-doped systems, the superconducting phase appears right after the semiconductor-metal transition, with increasing lead or potassium concentration.

\section{I.3.2 Normal-State Properties}

Much more experimental data are available for $\mathrm{BaPb}_{1-x} \mathrm{Bi}_{x} \mathrm{O}_{3}$ [2, 6, 7, 28, 30, 31, 34, 35, 36, 37, 38, 39, 40, 41, 42, 43 than for $\mathrm{Ba}_{1-x} \mathrm{~K}_{x} \mathrm{BiO}_{3}$. Some properties of the metallic phase $(0<x<0.35)$ of $\mathrm{BaPb}_{1-x} \mathrm{Bi}_{x} \mathrm{O}_{3}$ are given in Table I.1: Resistivities are quite high, nearly independent of temperature, and increase from $210 \mu \Omega \mathrm{cm}$ ( at $x=0$ ) to $540 \mu \Omega \mathrm{cm}$ ( at $x=0.24$ ). Carrier concentrations, obtained from measurements of the Hall coefficient 38, 39] range from $1.42 \times 10^{20} \mathrm{~cm}^{-3}($ at $x=0)$ to $2 \times 10^{21} \mathrm{~cm}^{-3}$ ( at $x=0.2$ ); the effective mass at $x=0.2$ is $0.3 m_{e}$ (obtained from plasma-edge data [37, 43]). The specific heat $C$ depends linearly on $T$ at low temperature, but the coefficient of proportionality is rather low [24, 39] ( $\simeq 0.6 \mathrm{~mJ} /$ mole $\mathrm{K}^{2}$ at $\left.x=0\right)$. However, plots of $C / T$ vs $T^{2}$ show some deviation from linearity (requiring fits of the form 39 $C=\gamma T+\beta T^{3}+\alpha T^{5}$, with $\gamma=0.6 \mathrm{~mJ} /$ mole $\mathrm{K}^{2}, \beta=0.42 \mathrm{~mJ} / \mathrm{mole} \mathrm{K}^{4}$ and $\alpha=1.1 \mathrm{~mJ} / \mathrm{mole}^{6}$ ). In the range $0<x<0.2$ optical-reflectivity data can be fit to a Drude form as expected in a conventional metal. Plasma frequencies, as obtained from optical experiments 443, increase with increasing $x$ (i.e., with increasing Bi concentration) in the above range; this is consistent with the increase in carrier concentration with increasing $x$ (Table I.1), as

obtained from Hall-effect measurements. LAPW, band-structure calculations [3, 44, 45] are fairly successful in accounting for the observed properties of the metallic phase in the range $0<x<0.2$ (Sec.I.4); and the most recent band-structure calculations [46] have also obtained reasonable results for the insulating phase.

As the metal-semiconductor transition is approached by increasing $x$, the metallic 
phase starts showing unusual transport properties [2, 37, 39, 49] (see Sec.I.3.6): the temperature coefficient of resistivity becomes negative around $x=0.3$ (Fig.I.3a) even though, at low-enough temperatures, the system becomes superconducting for $x<0.35$, and only beyond $x=0.35$ is true semiconducting behaviour (i.e., an activated behaviour over a large temperature range with the low-temperature resistivity showing no sign of a superconducting transition) observed down to the lowest temperatures measured (Fig.I.3a and I.3b). Reflectivity data show a gradual departure from Drude fits [49] in the metallic phase from $x=0.25$ onwards as the metal-semiconductor boundary is approached (Fig.I.4).

Various transport and optical properties in the semiconducting phase of $\mathrm{BaPb}_{1-x} \mathrm{Bi}_{x} \mathrm{O}_{3}$ have been measured, but there is a dearth of such systematic data for the $\mathrm{Ba}_{1-x} \mathrm{~K}_{x} \mathrm{BiO}_{3}$ system, though some measurements have started coming out 47, 48. For $\mathrm{BaPb}_{1-x} \mathrm{Bi}_{x} \mathrm{O}_{3}$ the semiconducting phase extends over a wide range: $0.35<x<1$. The temperature dependence of the resistivity in this phase yields a transport-activation energy $E_{A}$ which goes from 0 at $x=0.35$, to $0.2 \mathrm{eV}$ at $x=0.6$, and finally to $0.24 \mathrm{eV}$ at $x=1$ (Fig.I.5). By combining the resistivity and Hall-coefficient data, the temperature dependence of the carrier concentration in $\mathrm{BaBiO}_{3}$ (i.e., $x=1$ ) is found to be $n(T)=1.1 \times 10^{22}\left(\mathrm{~cm}^{-3}\right) \exp \left(-0.24 \mathrm{eV} / k_{B} T\right)$. The pre-exponential factor is remarkably large [2] and nearly equal to the number of unit cells per $\mathrm{cm}^{3}$, suggesting that the transport mechanism in this system is most probably intrinsic (and not extrinsic, i.e., not because of dopants, impurities, or defects).

Recently some measurements of the resistivity of single crystals of $\mathrm{Ba}_{1-x} \mathrm{Ki}_{x} \mathrm{BiO}_{3}$ have been carried out in the semiconducting phase [50, 51] for different values of $x$. Hellman, et al. [50] fit their resistivity data for single-crystal and thin-film samples of $\mathrm{Ba}_{1-x} \mathrm{Ki}_{x} \mathrm{BiO}_{3}$ to a variable-range hopping law $\rho=\rho_{0} \exp \left[\left(T_{0} / T\right)^{1 / 4}\right]$, with a very high $T_{0}\left(\simeq 3-6 \times 10^{8} \mathrm{~K}\right)$. According to them the states are very strongly localized and there is no indication of any nonlinear transport in these systems, so incommensurate CDW motion is possibly ruled 
out. Resistivity data [52 for superconducting $\mathrm{Ba}_{1-x} \mathrm{Ki}_{x} \mathrm{BiO}_{3}$ ceramics are also becoming available. These measurements seem to indicate [52] that there is a variable-range-hopping regime with activation energy $T_{0} \simeq 10^{4}-10^{6}$ in the normal state. It is now being argued by several groups [50, 51] that, even in single-crystal samples of $\mathrm{Ba}_{1-x} \mathrm{Ki}_{x} \mathrm{BiO}_{3}$, two or more phases with different chemical compositions are present. (If so, then presumably one phase is metallic and the other semiconducting.)

Sato et al., [48 have carried out a series of transport measurements on $\mathrm{Ba}_{1-x} \mathrm{Ki}_{x} \mathrm{BiO}_{3}$ thin films at $x=0.45$ and found no evidence of contributions from impurities or inhomogeneous distribution of potassium concentration in their annealed samples. The resistivity data for the best samples have a positive (but small) slope. Metallic temperature dependence is observed down from $\mathrm{T}_{C}$ upto $300 \mathrm{~K}$ and the resisitivity at $300 \mathrm{~K}$ is lower than $500 \mu \Omega \mathrm{cm}$. On the other hand negative temperature coeffiecient of resistivity was observed in samples that were grown at higher annealing temperature and these samples have resistivities much higher than $700 \mu \Omega \mathrm{cm}$.

The Hall coefficient measurement [48] on $\mathrm{Ba}_{1-x} \mathrm{Ki}_{x} \mathrm{BiO}_{3}$ thin films indicates that the charge carriers are electrons. This is in contradiction to the expectation that the carriers should be holes as monovalent potassium replaces divalent barium in $\mathrm{Ba}_{1-x} \mathrm{Ki}_{x} \mathrm{BiO}_{3}$. The value of Hall coeffiecient $\mathrm{R}_{H},(-) 3 \times 10^{4} \mathrm{~cm}^{3} / \mathrm{C}$ is about two times smaller than that expected from band structure calculations. The temperature dependence of $\mathrm{R}_{H}$ is quite small. These workers have also done Raman measurements and found that the height of the strong peak at $570 \mathrm{~cm}^{-1}$ that one observes at $x=0$, is reduced and it broadens with potassium doping. This peak disappears close to the metal-insulator transition. The reduction of the peak as well as its broadening with doping is considered by these workers to be an indication that the remnant CDW like ordering that one finds in the metallic phase of the lead doped system [49, 55] (close to the metal-insulator transition) is absent in the potassium doped system. 
Blanton, et al. [47] have measured the reflectivity spectrum of polycrystalline samples of $\mathrm{Ba}_{1-x} \mathrm{Ki}_{x} \mathrm{BiO}_{3}$ as a function of doping $x$ from $x=0$ to $x=0.4$ in the frequency range from $\simeq 250$ to $25000 \mathrm{~cm}^{-1}$. Their data on optical conductivity (obtained from the reflectivity data) indicate that the CDW conductivity peak at $16000 \mathrm{~cm}^{-1}$ at $x=0$ shifts downwards in energy and broadens substancially with doping. The spectrum at $x=0.4$ shows a broad contribution to the conductivity that is centred around $4000 \mathrm{~cm}^{-1}$. Blanton, et al. claim that this broad peak, has evolved from excitations across the single-particle (CDW) gap of $\mathrm{BaBiO}_{3}$ and is an indication that some remnant CDW order persists even in the metallic phase close to the transition as in the case of lead-doped barium bismuth oxide [49, 55]. Bozovic, et al. [53] have measured the far-infrared reflectance spectra in thin film samples of the superconducting $\mathrm{Ba}_{1-x} \mathrm{Ki}_{x} \mathrm{BiO}_{3}$ at $x=0.4$. They point out some overall similarity between the reflectance spectra of the cuprate superconductors and the bismuthates at optimal doping (corresponding to the highest $\mathrm{T}_{c}$ ). All these spectra are nearly linear in frequency $R(\omega) \simeq A-B \omega$ (where $A, B$ are constants, $A \approx 1, B / \hbar \approx 1$ $\mathrm{eV}^{-1}$ ) below the plasma edge.

\section{I.3.3 Charge Disproportionation and CDW ordering}

The actual valence state of $\mathrm{Bi}$ in the barium bismuthates is a matter of some dispute (Sec.I.1). Evidence in favour or against valence skipping by Bi in these systems is indirect. In particular, there has been some controversy in the experimental literature concerning the presence of a CDW in the semiconducting phase of these compounds [2, 6, 7, 27, 28]. The presence of the two valence states, $\mathrm{Bi}^{3+}$ and $\mathrm{Bi}^{5+}$, is intimately connected to this question. Many consider that the controversy has been settled more or less in favour of a CDW in the lead-doped system, but the results for the potassium-doped system are not clear yet. It is important to note that, to support a CDW in these systems, the charge contrast need not necessarily be $2 e$ [6, 7]; indeed it is almost certainly less than $2 e$, as 
suggested by recent, high-energy-spectroscopy experiments [27. The CDW controversy in these systems is complicated further by the methods used for sample preparation [6, 7]: oxygen vacancies appear quite frequently in samples prepared in low-oxygen atmospheres, leading to inhomogeneities that make it difficult to identify long-range CDW ordering experimentally. Even in the lead-doped system, it is still not clear how the CDW order disappears with increasing inhomogeneity (or whether there is short-range CDW order away from perfect stoichiometry). Optical-reflectivity spectra show that the CDW is quite robust (see below) and persists throughout the semiconducting regime (upto a lead concentration of $65 \%)$.

Neutron-diffraction (powder) studies [8, 29] of $\mathrm{BaK}_{1-x} \mathrm{Bi}_{x} \mathrm{O}_{3}$ show that the frozen, breathing-mode distortion of the oxygen octahedra leads to long-range CDW order in some of the semiconducting phases of $\mathrm{BaK}_{1-x} \mathrm{Bi}_{x} \mathrm{O}_{3}$, but not in the semiconducting phase abutting the superconducting phase; so the cause of the semiconducting nature of this phase remains a mystery [8, 10, 29].

\section{I.3.4 Optical Properties}

A number of optical-reflectivity measurements [2, 28, 43, 49] have been performed on the lead-doped system, both in the metallic and semiconducting phases, covering a wide range of frequencies, from far-infrared to visible. The metallic regime shows a characteristic Drude behaviour with a plasma edge, which shifts continuously towards high frequencies with increasing $x$. This edge is seen in the entire composition range without an abrupt change anywhere. Such a continuous variation of the reflectivity edge with $x$ is indicative of the homogeneity of the system upto optical length scales. In the semiconducting phase the reflectivity spectra are dominated by low-frequency optical phonons. No discontinuous change is observed anywhere in the spectra in the metalsemiconductor transition regime. 
In optical-conductivity spectra, obtained from reflectivity data, deviations from Drude behaviour occur as the transition is approached from the metallic side. This transition is marked by the transfer of the spectral weight into a high-frequency, quasi peak [2, 28, 54, 55], which grows with increasing $x$ into a peak containing most of the spectral weight in the semiconducting phase. The position of this peak shifts towards higher frequencies with increasing $x$ as shown in Fig.I.6a. (Fig.I.5 shows this peak position as a function of $x$ ). It has been suggested [2] that this peak (Fig.I.6a,b) has a profile which is like that of a CDW system with a gap along the entire Fermi surface (as in $\mathrm{K}_{0.3} \mathrm{MoO}_{3}$ ) and not like the square-root absorption edge in conventional semiconductors. However, more recent work shows a broader peak [47, 60]. The magnitude of the gap $E_{G}$, measured by the position of this peak in the optical-conductivity spectrum, is an order of magnitude higher than $E_{A}$, the transport activation energy [2, 9, 10], e.g., $E_{G}=2.0 \mathrm{eV}$ and $E_{A}=0.24$ $\mathrm{eV}$ at $x=1$, whereas in a conventional semiconductor $E_{A}=E_{G} / 2$. The optical gap decreases with doping (Figs. I.5 and I.6) and, at the semiconductor-metal transition, it is quite small but nonzero. (The transport activation energy vanishes at the transition.) On the metallic side the plasmons show up strongly in the reflectivity spectra and mask the details of the optical gap. Throughout the semiconducting phase the optical gap is larger than twice the transport gap. The transport activation energy or gap $E_{A}$ does not show up clearly in any optical, photoconductivity, or photoacoustic studies.

Raman experiments [28, 56] on $\mathrm{BaBiO}_{3}$ show resonant enhancement and the appearence of higher harmonics of the $569 \mathrm{~cm}^{-1}$ mode phonon (the breathing mode described above), indicating that the optical gap $E_{G}$ is related to the strong, electron-phonon interaction in the system [28, 30, 31, 57, 58, 59, 94, 96]. The resonant phonon energy for the potassium-doped system shows very similar behaviour and, as in the lead-doped case, the resonance decreases with increasing $x$. So far no one has reported any observation of two distinct peaks in the Raman intensity which could be identified with the two valence states of $\mathrm{Bi}$ directly. If there were a strong charge disproportionation in $\mathrm{BaBiO}_{3}$, one 
would have observed a split peak with equal intensities. It is possible that the laser power used in these experiments creates oxygen vacancies and destroys the charge disproportionation locally. We have not come across any discussion of these issues in the experimental literature.

\section{I.3.5 Magnetic Properties}

Both the lead- and potassium-doped systems are diamagnetic in their normal phases over the entire temperature range of measurement and for all values of doping. The diamagnetic contribution from the core, which constitutes almost the entire susceptibility in both the systems, when subtracted from the total susceptibility, leaves a tiny paramagnetic part which, in the lead-doped system, shows a somewhat unusual behaviour: it increases [2] as the system becomes more insulating, over the entire range of $x$ (Fig.I.7a). For the potassium-doped system the paramagnetic contribution remains nearly independent of $x$ [2, 6, 7, 21, 22, 23, 24. The paramagnetic contribution from the valence electrons is very small and lies between $\approx 0.8$ to $2 \times 10^{-5} \mathrm{emu} / \mathrm{mol}$ in barium potassium bismuth oxide. The temperature dependence of magnetic susceptibility is shown in Fig.I.7b.

\section{I.3.6 Metal-Semiconductor Transition}

The insulating nature of $\mathrm{BaBiO}_{3}$ and the metal-semiconductor transition that occurs on doping with $\mathrm{Pb}$ or $\mathrm{K}$ cannot be understood on the basis of band-structure calculations [3, 44, 46]: they yield a half-filled conduction band for $\mathrm{BaBiO}_{3}$. The simplest explanation for the insulating nature of $\mathrm{BaBiO}_{3}$ invokes a CDW instability which opens a gap in the conduction band (see below). Both $\mathrm{Pb}$ and $\mathrm{K}$ doping should inhibit CDW ordering for two reasons: first, such doping moves the Fermi level down from its position at half filling (K doping removes electrons from the system; for $\mathrm{Pb}$ doping this follows from band-structure calculations); and it also introduces disorder. 
We now summarize the experimental findings close to the metal-semiconductor transition in the $\mathrm{BaPb}_{1-x} \mathrm{Bi}_{x} \mathrm{O}_{3}$ system. There is a strong precursor effect [63] of the metalsemiconductor transition as the $\mathrm{Bi}$ concentration is increased beyond $x=0.2$. The resistivity data are shown in Figs. I.3a and I.3b. The temperature coefficient of resistivity becomes negative for $x \lesssim 0.2$. This would normally indicate that the system is already insulating. However, at low temperatures, the resistivity drops to zero upto $x=0.35$ (Fig.I.3a), indicating a superconducting transition. Although the resistivity (for $0.25 \lesssim x \lesssim 0.35$ ) decreases with increasing temperature, it cannot be fitted to an activated behaviour over a wide temperature range. Such a fit is possible only beyond $x=0.35$ (Fig.I.3b) where, down to the lowest temperatures measured, no superconducting transition is observed. Hence the criteria used by experimentalists [2] for the metalsemiconductor transition in these systems are the absence of superconductivity at low temperatures and an activated behaviour of the resistivity over wide temperature ranges. There is then really no clear separation between the insulator and metal in the transition region [81] and we might have a transition from a (Anderson-localized ?) semiconductor to a superconductor.

Reflectivity spectra (Fig.I.4) show a marked departure from Drude behaviour from $x=0.2$ onwards. The temperature dependence of the resistivity is unusual: From $x=0.2$ the resistivity becomes temperature independent and around $x=0.25$ its temperature coefficient changes sign. The system is, however, metallic at that concentration (the resistivity does not show an activated behaviour). The thermopower varies linearly with temperature for $x<0.2$; for $x>0.2$ it no longer follows this metallic behaviour.

Reflectivity spectra do not show any abrupt change across the metal-semiconductor transition in the high-frequency region. In the semiconducting phase there are several optic phonons in the low-frequency region. As the metal-semiconductor transition is approached, the low-frequency conductivity is reduced and the spectral weight is transferred 
to the high-frequency quasi peak as discussed earlier. The quasi peak has a tail extending into the low-frequency regime indicating the possibility of states in the semiconducting energy gap. This tail disappears completely beyond $x=0.7$.

\section{I.3.7 Superconducting Properties}

The superconducting phase occurs [1, 2, 4, 5, 6, 7] in the concentration range $0.05<$ $x<0.30$ for $\mathrm{BaPb}_{1-x} \mathrm{Bi}_{x} \mathrm{O}_{3}$ and $0.37<x<0.60$ for $\mathrm{Ba}_{1-x} \mathrm{~K}_{x} \mathrm{BiO}_{3}$. The highest $T_{c}$ is $13 \mathrm{~K}$ at $x=0.25$ for the former and $34 \mathrm{~K}$ at $x=0.4$ for the latter . The densities of states

at the Fermi level for both these bismuthate systems are quite low [15, 24, 39]. Given such meagre densities of states at the Fermi levels and the three-dimensional structures of these bismuthates, the superconducting transition temperatures are very high indeed. The specific-heat anomaly associated with the superconducting transition is very small [30, 31, 39]. Tunneling [24, 64, 65] and infrared reflectivity measurements 64, 65] yield $2 \Delta_{s} / k_{B} T_{c} \simeq 3.5$, the BCS value, where $\Delta_{s}$ is the superconducting energy gap (measured from the chemical potential). For both these bismuthates there is a substantial isotope effect with $T_{c} \propto M^{-\alpha}$, and $\alpha=0.2-0.3$ [24] (A higher value of nearly 0.4 has been reported by Hinks, et al. [14, 15], for the potassium-doped system.). The superconducting properties of these systems are summarised in Table I.2. Note especially the coherence lengths $\left(\xi_{G L}\right)$ which are nearly $70-80 \AA$, i.e., about $30-40$ lattice spacings [18, 24].

Sato et al. [48] have measured the superconducting properties of $\mathrm{Ba}_{1-x} \mathrm{Ki}_{x} \mathrm{BiO}_{3}$ (at $x=$ 0.45) thin film annealed samples. Infrared reflectivity spectrum in the superconducting state at $5 \mathrm{~K}$ (normalized by its value in the normal state at $30 \mathrm{~K}$ ) shows a clear peak at $60 \mathrm{~cm}^{-1}$ and a broad dip at $135 \mathrm{~cm}^{-1}$. The same features were earlier obtained by Schlesinger et al. [65] in high density cintered samples with a transition width of 4K. The superconducting energy gap that Sato et al. obtain is $3.7 \pm 0.3 \mathrm{meV}$, in good agreement with Schlesinger et al. With the superconducting transition temperature of 
their sample being $22.5 \mathrm{~K}$, the value of $2 \Delta / k_{B} T_{c}$ is about 3.8. Similar values have also been reported in the optical measurement [65], point contact tunnelling measurement 67 on $\mathrm{Ba}_{1-x} \mathrm{Ki}_{x} \mathrm{BiO}_{3}$ sintered samples at $x=0.4$ and tunnel junctions fabricated on thin films grown by molecular-beam-epitaxy [68] and laser deposition method [69]. The conductance spectra for several tunnel junctions have been measured [48, 72] from 10 to $100 \mathrm{mV}$ bias voltage. The conductance spectrum in the region greater than $25 \mathrm{mV}$ has no observable phonon structure [48, 72]. This result seems to suggest that there is no strong electron-phonon coupling as has been assumed by many workers [94.

The upper critical field in the barium potassium bismuthates has been measured in thin films of polycrystalline samples [48] and in sintered samples [70, 71]. Unlike in the case of sintered samples, the thin films show a reasonably sharp resistive transition in a magnetic field. For the best thin film samples 448 $\mathrm{H}_{c 2}$ shows almost linear temperature dependence down to $4.2 \mathrm{~K}$ and seems to saturate aound $1.7 \mathrm{~K}$.

The upper critical field $\left(\mathrm{H}_{c 2}\right)$ measurements in polycrystalline samples of $\mathrm{Ba}_{1-x} \mathrm{~K}_{x} \mathrm{BiO}_{3}$ by Crabtree [66], and Filippini et al., [66] show that there is a significant upward curvature at fields beyond 10 Tesla. There is also noticeable flattening of the $\mathrm{H}_{c 2}$ curve close to the superconducting transition temperature. The value of the temperature coeffiecient of $\mathrm{H}_{c 2}$ at the transition temperature $\left(d H_{c 2} / d T\right.$ at $\left.T_{c}\right)$ for $\mathrm{Ba}_{1-x} \mathrm{~K}_{x} \mathrm{BiO}_{3}$ presented in Table I.2 $(\simeq-0.5 T / K)$ is for polycrystalline samples (from Batlogg, et al., 24). Measurements on thin films 48 indicate a much larger value of nearly $-1.0 \mathrm{~T} / \mathrm{K}($ at $x=0.45)$.

Hysteretic Josephson junction behavior has been observed in the $I-V$ characteristics of multidomain films of $\mathrm{Ba}_{1-x} \mathrm{Ki}_{x} \mathrm{BiO}_{3}$ at temperatures upto $8 \mathrm{~K}$ by Martin et al., 73 . They fit this behaviour to a model of Josephson junctions in series coupled by a shunt capacitor.

\section{I.3.8 The Effect of Oxygen-Deficiency on the Properties of $\mathrm{BaPb}_{1-x} \mathrm{Bi}_{x} \mathrm{O}_{3}$}


The effect of oxygen deficiency on the structural and transport properties of the leaddoped system has been studied by a few groups [63, 74, 75, 76, 77. Oxygen vacancies are easily created by sputtering in a thin film of a single-crystalline $\mathrm{BaPb}_{1-x} \mathrm{BiO}_{3}$ sample. The principal effect of an increase in oxygen vacancies is to increase the resistivity in the metallic regime and decrease the superconducting $T_{c}$. Furthermore the resistivity of an oxygen-deficient metallic sample of $\mathrm{BaPb}_{1-x} \mathrm{Bi}_{x} \mathrm{O}_{3}$ (at $x=0.3$ ) goes down with increasing temperature and the sharpness of this fall in resistivity increases with increasing oxygen deficiency (Fig.I.8a). Thus, at a fixed temperature, as the oxygen deficiency increases, so does the resistivity. However, the "carrier density", as inferred naively from the Hall coefficient (for such a strongly disordered system), increases with oxygen deficiency (Fig.I.8b). The increase in carrier density with oxygen deficiency is thus not reflected in the resistivity results. Hence this suggests, 63 that there is a strong carrier localization effect because of the defects created by the missing oxygen atoms, which more than offsets the increase in "carrier density".

It is surprising that the carrier density should increase with oxygen deficiency. For, as more and more oxygen is removed from the system, so are electrons, and one would expect a decrease in the carrier (electron) density (the Hall coefficient is negative). At very low temperatures (Fig.I.8b) it does seem that the more oxygen deficent samples have lower carrier densities; however, this trend is reversed at high temperatures. Enomoto, et al. [63] argue that, at high temperatures, the carriers that were localized by the oxygen deficiency are delocalized (the activation energy for localization that fits their assumption is quite small $\simeq 18 \mathrm{meV}$ ) and give rise to the enhanced carrier density; but the resistivity data, (Fig.I.8b) do not reflect this enhanced carrier density! This clearly calls into question the naive interpretation of the Hall-effect data.

Another interesting observation 63 is that annealing a sample of the lead-doped system with $x=0.35$ at $873 \mathrm{~K}$ and 1400 atm. (in an atmosphere of $80 \% \mathrm{Ar}_{2}$ and $20 \%$ 
$\mathrm{O}_{2}$ ) for 8 hours changes it from a semiconductor to a superconductor. This high-pressure annealing decreases the resistivity markedly and, at $6 \mathrm{~K}$, there is a sharp superconducting transition with a width less than $1 \mathrm{~K}$. However, if the sample is annealed at a pressure of 1 atm. (in an oxygen atmosphere) and at $600 \mathrm{~K}$, it shows no superconductivity. This indicates that a metal-semiconductor transition can be induced in the lead-doped system by increasing the number of $\mathrm{O}$ vacancies. The sharpness of the superconducting transition is also greatly enhanced as the $\mathrm{O}$ deficiency decreases. A positron - annihilation study 777 on single-crystal samples of $\mathrm{Ba}_{1-x} \mathrm{~K}_{x} \mathrm{BiO}_{3}$ in the range $15 \mathrm{~K}$ to $300 \mathrm{~K}$ shows that the sharpness of the superconducting transition and the value of the transition temperature depends strongly on oxygen vacancies, increasing as one approaches stoichiometry. There is no signature of positron localization and the positron lifetime is determined by the oxygen stoichiometry.

A naive interpretation of these results [63] is most probably fallacious. Firstly, there should be strong Anderson-localization effects in the electronic band, for, apart from the $\mathrm{O}$ vacancies, the concentration of $\mathrm{Bi}$ is about $30 \%$. Secondly, the experimental system (at $x=0.3$ ) is quite close to its metal-semiconductor transition and, as we saw earlier, this region is quite unusual. In particular, it is not clear whether the samples used in these experiments were metallic or semiconducting. Thus a more careful analysis of these results along with more controlled measurements are clearly necessary to understand the unusual observations reported here.

\section{I.4 The Electronic Structure of Barium Bismuthates}

The electronic structure of the barium bismuthates has been studied well using detailed band-structure calculations [3, 44, 46] as well as various experimental methods [28]. The electronic configuration of a $\mathrm{Bi}$ atom is $[\mathrm{Xe}] 4 f^{14} 5 d^{10} 6 s^{2} 6 p^{3}$ and that of a $\mathrm{Pb}$ atom is $[\mathrm{Xe}] 4 f^{14} 5 d^{10} 6 s^{2} 6 p^{2}$. Hence in the $4^{+}$valence state (which is the nominal valence state of 
$\mathrm{Bi}$ and $\mathrm{Pb}$ in these bismuthates) $\mathrm{Bi}^{4+}$ has an open-shell configuration $6 \mathrm{~s}^{1}$, whereas $\mathrm{Pb}^{4+}$ has a closed outer-shell configuration.

In both the lead- and potassium-doped systems, each $\mathrm{Bi}$ atom is surrounded by six $\mathrm{O}$ atoms which form an octahedron (Fig.I.1a). There are ten orbitals in the "valence band" of the bismuthates [44, 45] given the $\mathrm{BiO}_{3}$ unit cell, each $\mathrm{O}$ atom contributes three $2 \mathrm{p}$ orbitals, and each Bi atom contributes one 6s orbital (Fig.I.10). The Bi-O bond lengths are the same for all the six $\mathrm{O}$ atoms in the cubic phase. We first examine the electronic structure of the cubic phase and later discuss the effects of the noncubic distortions.

\section{I.4.1 The Electronic Structure of $\mathrm{BaPb}_{1-x} \mathrm{Bi}_{x} \mathrm{O}_{3}$}

The electronic band structure of the bismuthates (Figs.I.11a - I.11c) has been computed by using the LAPW method 44, 45. The flat, low-lying bands are derived from $\mathrm{Ba} 5 \mathrm{p}$ states and the uppermost complex of unoccupied states are derived from $\mathrm{Ba} 5 \mathrm{~d}$ and Bi 6p states. Note that the bands derived from Barium are admixed only marginally with the valence-band manifold in the middle.

Of the three $2 \mathrm{p}$ orbitals at each $\mathrm{O}$ atom, one forms a $\sigma$ bond (Fig.I.10) with the Bi/Pb 6s orbital (spherically symmetric) and the rest form $\pi$ bonds. The $\pi-\pi^{*}$ manifold falls within the $\sigma-\sigma^{*}$ manifold (Figs.I.11 and I.12). The Fermi level crosses the uppermost $\sigma$ antibonding subband (Fig.I.11), leaving it exactly half filled for $\mathrm{BaBiO}_{3}$ (Fig.I.11c). As Bi is replaced by $\mathrm{Pb}$, the filling of this $\sigma^{*}$ subband changes (Fig.I.11a-b). In addition, LAPW band-structure calculations show that $\mathrm{Pb}$ doping also introduces a noticeable chemical shift in the position of the 6 s states. In all cases the $\mathrm{Ba}$ bands are well away from $E_{F}$. The principal features of these bands can be understood in terms of a tight-binding model 44, 45. Figure I.13a shows such a fit with a three-parameter, tight-binding calculation. The three parameters used are the orbital energies $\epsilon_{6 s}=-4.1 \mathrm{eV}, \epsilon_{2 p}=-1.9 \mathrm{eV}$ and the

nearest-neighbour, Bi-O hopping parameter (along the $\sigma$ bond) $t_{s p \sigma}=2.2 \mathrm{eV}$. As there is 
no overlap in this model between $\mathrm{Bi} 6 \mathrm{~s}$ and $\mathrm{O} 2 \mathrm{p}_{\perp}$, the entire $\pi-\pi^{*}$ manifold now shrinks to a set of perfectly flat and degenerate nonbonding bands. A five-parameter fit improves upon this, but the three-parameter fit clearly shows the tight-binding, $\sigma$ character of the important bands. We can, therefore, use this set of parameters to study the low-energy physics of these systems. This three-parameter, tight-binding model also predicts an electron-like, $\Gamma$-centered, simple-cubic Fermi surface for $\mathrm{BaBiO}_{3}$. The electron surface nests perfectly with an identical hole surface centered at the zone corner (R) (Fig.I.13b).

Such a nested Fermi surface is susceptible to an electronic instability that, in the bismuthates, leads to a Peierls distortion, in which the $\mathrm{O}$ atoms surrounding every alternate Bi site are displaced towards them. This is the breathing-mode displacement of the oxygen octahedra, which doubles the unit cell and thereby opens up a gap along the entire, noninteracting Fermi surface, splitting the $\sigma^{*}$ subband into a filled and an unfilled band. The effect of this Peierls distortion is shown, in a folded-zone scheme in Fig.I.14 for only the conduction-band states near $E_{F}$, using the three-parameter, tight-binding model. This gap is estimated to be nearly $1 \mathrm{eV}$ in a cubic $\mathrm{BaBiO}_{3}$ structure, with alternate Bi-O bond lengths differing by $0.16 \AA$ [4]. This lowering of the energies of the states near $E_{F}$ comes entirely from the Bi-O bond alteration and hence stabilizes such a distortion. From the point of view of band-structure calculations, the role of doping is merely to inhibit the frozen-in commensurate distortion (since the Fermi level moves away from nesting), thereby producing a metal in which the conduction electrons are strongly coupled to the dynamic oxygen, breathing-mode phonons.

Band-structure calculations work fairly well in the metallic regime of $\mathrm{BaPb}_{1-x} \mathrm{Bi}_{x} \mathrm{O}_{3}$ $(0<x<0.2)$. The band crossing the Fermi level is derived from Bi/Pb 6s-O $2 \mathrm{p}$ levels. Since Bi atoms supply one extra electron per atom to the conduction band, an increase in the Bi concentration pushes up the Fermi level and increases the number of carriers in the conduction band. The plasma edge shifts towards higher frequencies in this range exactly 
as predicted by the band-structure calculations. The observed low density of states (DOS) at $E_{F}$ is also brought out by calculations as the s-p character of the conduction band implies a low DOS at $E_{F}$ (see below). The Fermi surface for $x<0.2$ is nearly spherical as predicted by LAPW calculations. However, the band-structure results deviate from experimental observations from $x>0.2$ onwards and cannot produce the semiconducting phase for $x>0.35$.

\section{I.4.2 The Electronic Structure of $\mathrm{Ba}_{1-x} \mathrm{~K}_{x} \mathrm{BiO}_{3}$}

Band-structure (LAPW) results [3] are available for this system at various values of $x$. Figure I.15a shows these for $\mathrm{BaBiO}_{3}$ using the observed lattice parameters of $\mathrm{Ba}_{0.6} \mathrm{~K}_{0.4} \mathrm{BiO}_{3}$. The results are in close agreement with the ones obtained for the leaddoped system. The Fermi level crosses the uppermost of the ten-band, Bi 6s-O 2p complex. The flat, low-lying bands are Ba 5p states, whereas the uppermost complex of unoccupied states is derived from $\mathrm{Ba} 5 \mathrm{~d}$ and $\mathrm{Bi} 6 \mathrm{p}$ states. The manifold of $\mathrm{Bi} 6 \mathrm{~s}-\mathrm{O} 2 \mathrm{p}$ bands features a pair of broad $(\simeq 16 \mathrm{eV}) \sigma$ and $\sigma^{*}$ subbands extending from $-12 \mathrm{eV}$ to $+4 \mathrm{eV}$ at the zone corner. The $\sigma^{*}$ band is half filled; so, as a $\mathrm{K}^{1+}$ ion replaces a $\mathrm{Ba}^{2+}$ ion and removes an electron from the system, the Fermi level starts coming down the $\sigma^{*}$ band, and the occupancy of this band is reduced to $1-x$. Here also a three-parameter, tight-binding fit (similar to the $\mathrm{Pb}$-doped case) works quite well.

Recently Liechtenstein, et al. [46], have carried out what is perhaps the best bandstructure study of $\mathrm{Ba}_{1-x} \mathrm{~K}_{x} \mathrm{BiO}_{3}$. They use the LDA and the full linear muffin-tin-orbital method. For $x=0$ they find a lattice instability with a combination of both tilt $\left(u_{t}\right)$ and breathing $\left(u_{b}\right)$ distortions. This yields the experimentally observed monoclinic structure. For $x=0.5$ they correctly find the cubic phase, with $u_{t}=u_{b}=0$, to be stable.

An interesting point that emerges from this band-structure study is that, when the

details are put in, $\mathrm{BaBiO}_{3}$ may have a rather small indirect band gap (Fig.I.15b). The 
actual value of this gap, gleaned from Fig.I.15b, is roughly $0.025 \mathrm{Ry}$ or $0.34 \mathrm{eV}$; though this may not be very reliable. Hence one must consider the possibility that the small transport gap $(0.24 \mathrm{eV})$ reflects this aspect of the band structure. However, optical experiments must be able to see this as a phonon-assisted threshold, starting around $0.68 \mathrm{eV}$, in order to be consistent with the transport gap. All the data that we have seen have thresholds starting at $1 \mathrm{eV}$. (For recent spectroscopic data see Ref. [60.) If the latter is taken as the indirect gap, the discrepancy with the transport gap is still too large to be understood in conventional terms. (For example, if one were to attribute it to polaronic or bipolaronic effects, as some groups have done [2, 60, 61], the resulting Franck-Condon factors would be too large, leading to self-trapped rather than mobile carriers as required by the observed mobilities.) Thus one clearly needs an electronic negative- $U$ mechanism of the sort we have discussed elsewhere [9, 10] and which we will discuss below. (Of course, for a proper comparison with experimental data and a better estimate of model parameters it seems crucial to include the details of the band structure.)

\section{I.4.3 Comparison Between the Electronic Structures of Bismuthates and High- $T_{c}$ Cuprates}

It is interesting to compare the electronic structures of the high- $\mathrm{T}_{c}$ cuprates and bismuthates. There are some similarities [9, 45] which become apparent when viewed against the electronic structures of conventional 'low- $T_{c}$ ' oxide superconductors, such as TiO. There are also very significant differences between the two classes of high- $T_{c}$ oxide superconductors. Hence a comparison between their electronic structures and the resulting types of ordering (spin or charge) helps us in understanding the microscopic interactions that lead to the observed properties of these systems.

Both these high- $T_{c}$ superconductors are characterized by strongly hybridized bands ( $\mathrm{Cu} 3 \mathrm{~d}-\mathrm{O} 2 \mathrm{p}$ for the cuprates or $\mathrm{Bi} / \mathrm{Pb} 6 \mathrm{~s}-\mathrm{O} 2 \mathrm{p}$ for the bismuthates), in which the Fermi 
level falls within the uppermost $\sigma$ antibonding subband (Fig.I.12a-b), making it nearly half filled. In the cuprates this band is formed by the $\sigma$ bonding between $\mathrm{Cu} 3 \mathrm{~d}\left(x^{2}-y^{2}\right)$ and the $2 \mathrm{p}$ orbitals of the four surrounding oxygen atoms, and in the bismuthates, by the $\sigma$ bonding between the spherically symmetric and spatially extended $\mathrm{Bi} / \mathrm{Pb} 6$ s orbitals and the $2 \mathrm{p}$ orbitals of the six surrounding oxygen atoms. Note that, in low- $T_{c}$ oxides (e.g., TiO, $\mathrm{Li}_{1+x} \mathrm{Ti}_{2-x} \mathrm{O}_{4}$ ), the metal d states lie well above the O-2p manifold and $E_{F}$ falls within the $\pi-\pi^{*}$ manifold (Fig.I.12b). This has two effects: First, as the metal-3d orbitals are well above the O-2p orbitals (e.g., in TiO this separation is nearly $5 \mathrm{eV}$ ), the hybridization between the metal $\mathrm{d}$ and oxygen p orbitals is quite weak, so the states near $E_{F}$ for these low- $T_{c}$ materials are predominantly of $d$-character (which is consistent with the observation that oxygen stoichiometry has little effect on their electronic properties). Second, the $\pi-\pi^{*}$ mainfold being triply degenerate (formed by the hybridization between the triply degenerate $\mathrm{t}_{2 g}$ orbitals, viz., $\mathrm{d}_{x y}, \mathrm{~d}_{y z}$ and $\mathrm{d}_{z x}$, of the transition metal and the O-2p orbitals of the neighbouring oxygen atoms), the DOS at $E_{F}$, and hence the Sommerfeld constant $\gamma$, is much larger for them than for the high- $T_{c}$ oxide superconductors, where the $\sigma^{*}$ band is derived from the overlap of $\mathrm{O}-2 \mathrm{p}$ orbitals with nondegenerate $\mathrm{Cu} 3 \mathrm{~d}-x^{2}-y^{2}$ or $\mathrm{Bi} 6 \mathrm{~s}$ orbitals. The well-known $T_{c}$ vs. $\gamma$ curve for various superconductors (Fig.I.16) illustrates this feature: The cuprates and bismuthates have a very low DOS at $E_{F}$ compared to their superconducting $T_{c}$ 's.

We have already discussed that a tight-binding fit for the important bands works quite well for the bismuthates. In the cuprates a similar, tight-binding fit also works well. For $\mathrm{La}_{2} \mathrm{CuO}_{4} t_{d p \sigma}=1.8 \mathrm{eV}$ and $\epsilon_{d}=\epsilon_{p}=-3.2 \mathrm{eV}$; only two parametrs are required here as the $\mathrm{O}-2 \mathrm{p}$ and $\mathrm{Cu}-3 \mathrm{~d}$ levels are nearly degenerate in the tight-binding fit.

Like the bismuthates, the cuprates also have a nested Fermi surface (two dimensional as opposed to the three-dimensional one in the bismuthates) at zero doping. A system with a nested Fermi surface is susceptible to various instabilities like spin-density wave 
(SDW), CDW, etc. The nominal valence of $\mathrm{Bi}$ in the bismuthates is $4^{+}$and that of $\mathrm{Cu}$ in the cuprates is $2^{+}$, which means that $\mathrm{Bi}$ and $\mathrm{Cu}$ ions have just one electron in their outer shells in these compounds. Thus one should expect these oxides to show fully developed moments in their insulating states; however, these two systems show very different magnetic behaviour: The cuprates show a (somewhat reduced) moment whereas the bismuthates are perfectly diamagnetic and there is no indication of a moment (e.g., in ESR measurements). In addition, the insulating phase of the cuprates is antiferromagnetically ordered, but in the bismuthates it exhibits CDW ordering.

These differences suggest that the low-energy degrees of freedom in the cuprates are the spin degees of freedom, whereas those in the bismuthates are the charge degrees of freedom. Thus the dominant interaction in the cuprates is the onsite Coulomb repulsion (repulsive $U_{d d}$ ) between two electrons (or holes) at a $\mathrm{Cu}$ site and not the long-range part of the Coulomb interaction, which favours CDW ordering. (An effective (antiferromagnetic) spin model can be obtained from a strongly correlated electron system via the Anderson superexchange mechanism, if the correlation is repulsive.) However, in the bismuthates, CDW formation and the consequent quenching of the spin degrees of freedom suggests that the repulsive, onsite part of the Coulomb interaction is suppressed relative to the repulsive, long range Coulomb interaction. The suppression can arise from a strong electron-phonon coupling between the $\mathrm{Bi}$ atoms and the breathing mode of the surrounding $\mathrm{O}$ atoms [78, 79] or from an electronic mechanism [18]. There are reasonably convincing arguments (see below) for the onsite interaction in the bismuthates being effectively attractive and dominant relative to the nearest-neighbour, Coulomb repulsion. Thus, at the simplest level, the cuprates should be modelled by a Hubbard model with strong, onsite repulsion whereas the bismuthates should be modelled by using an extended Hubbard model with onsite attraction and nearest-neighbour repulsion.

The experimental overview presented above suggests that the bismuthates are qual- 
itatively different from both low- $T_{c}$, oxide superconductors and high- $T_{c}$, cuprate superconductors. Their superconduting and unusual normal-state properties make these bismuthates interesting, and an attempt to understand them a challenging task. So far there has been no fully successful theoretical treatment of these systems for all ranges of doping. However, there have been quite a few attempts, with varying degrees of success, to understand some of the experiments mentioned above as we discuss below.

\section{Model Hamiltonians}

In this Section we review the various models and theoretical treatments that have been put forth to understand some of the experimental properties of the barium bismuthates, so as to put our work in perspective. These theories typically concentrate on some subset of the available experimental observations. Furthermore, most of these theories assume that the important, low-energy physics of these bismuthates can be understood on the basis of a negative- $U$, extended Hubbard model with an attractive, onsite and a repulsive, nearest-neighbour, Coulomb interaction. An important issue, which has much bearing on a theoretical understanding of these systems, is the origin of the attractive, onsite interaction. Two points of view exist in the literature:

(1) that the attraction arises because of a strong electron-phonon coupling [78, 79]; and

(2) that the attractive interaction is electronic in origin 18.

We discuss these two points of view below and the theories that use them.

In Section II.1 we motivate and describe the one-band, negative- $U$, extended-Hubbard model for the bismuthates, and discuss the origin of the negative $U$. We also describe the (well-known) mapping of this model onto a pseudospin model in the strong- $U$ limit. In

Sec.II.2 we discuss some earlier theoretical treatments of these models and analyze them in the context of experimental results. 


\section{II.1 The One-Band, Negative- $U$, Extended Hubbard Model For The Bismuthates}

At the $\mathrm{BaPbO}_{3}$ end of the $T-x$ phase diagram of $\mathrm{BaPb}_{1-x} \mathrm{Bi}_{x} \mathrm{O}_{3}$ (i.e., in the region $x<0.2)$ most of the properties of these bismuthates are conventionally regarded as being consistent with the simple band picture of Mattheiss and Hamman (Sec.I.4). A wide conduction band is formed because of the hybridization of $\mathrm{Pb}$ (or $\mathrm{Bi}$ ) 6s orbitals with O$2 \mathrm{p}_{\sigma}$ bonding orbitals. An approximate, tight-binding characterization of this band may be made by choosing $E_{6 s}(B i / P b)=-4.1 \mathrm{eV}, E_{2 p}=-1.9 \mathrm{eV}$, and $t_{s p \sigma}=2.2 \mathrm{eV}$. The band is partially filled as $\mathrm{Pb}^{4+}$ contributes no conduction electrons to it (whereas $\mathrm{O}^{2-}$ contributes six p electrons per oxygen to the entire s-p band and two p electrons per oxygen to the bonding band). The superconducting transition temperature $T_{c}$ is low; however, it increases with $x$ in a manner that is consistent with the increase (with $x$ ) of both $N(0)$, the density of states at the Fermi level, and $\lambda_{e p}$, the electron-phonon coupling constant [56].

For the regime $x>0.2$, it is clear that such a simple band picture is inadequate because electron-electron and electron-phonon interaction effects are very important. It has been suggested [78, 79] that some of the essential features of these interaction effects can be incorporated in an effective, semi-phenomenological, multiband, negative- $U$, extendedHubbard model where only the $\mathrm{O}-2 \mathrm{p}_{\sigma}$ and the $\mathrm{Bi} / \mathrm{Pb}$ s orbitals are kept explicitly, namely,

$$
\begin{gathered}
\mathcal{H}_{m b}=-t_{s p \sigma} \sum_{<i j>\sigma}\left(c_{i \sigma}^{\dagger} p_{j \sigma}+H . C .\right)-U_{1} / 2 \sum_{i \sigma} \hat{n}_{i \sigma} \hat{n}_{i-\sigma}+\epsilon_{p} \sum_{i \sigma} p_{i \sigma}^{\dagger} p_{i \sigma} \\
+V_{1} \sum_{<i j>\sigma \sigma^{\prime}}\left(c_{i \sigma}^{\dagger} c_{i \sigma}\right)\left(p_{j \sigma^{\prime}}^{\dagger} p_{j \sigma^{\prime}}\right) .
\end{gathered}
$$

Here $c_{i \sigma}^{\dagger}$ creates Bi 6 s electrons at the $i$ sites of a cubic reference lattice (at this level of modelling, noncubic distortions are regarded as higher-order effects which can 
be incorporated at a later stage), $p_{j}$ is the destruction operator for oxygen electrons (in the $\sigma$ orbital), $t_{s p \sigma}$ is the hopping matrix element for the transfer of an oxygen electron from the $p_{\sigma}$ orbital to a nearest-neighbour bismuth (or lead) atom, $U_{1}$ and $V_{1}$ account for onsite and nearest-neighbour Coulomb interactions, respectively, and $\epsilon_{p}$ is the chemical potential for oxygen electrons (with respect to the bismuth 6s level).

It is easy to show [10, 18] that, in the limit $t_{s p \sigma} \ll \epsilon_{p}$, this reduces to a negative$U$, extended-Hubbard model involving the Bi atoms only (i.e., involving electrons in the Wannier orbitals, centered on Bi atoms, of the antibonding $\mathrm{Bi} 6 \mathrm{~s}-\mathrm{O} 2 \mathrm{p}_{\sigma}$ band only), given by the Hamiltonian

$$
\mathcal{H}=-t \sum_{<i j>\sigma}\left(c_{i \sigma}^{\dagger} c_{j \sigma}+\text { h.c. }\right)-U / 2 \sum_{i \sigma} \hat{n}_{i \sigma} \hat{n}_{i-\sigma}+V \sum_{<i j>\sigma \sigma^{\prime}} \hat{n}_{i \sigma} \hat{n}_{j \sigma^{\prime}}-\mu \sum_{i} \hat{n}_{i}
$$

where $<i j>$ are nearest-neighbour pairs of sites, $\hat{n}_{i}=\sum_{\sigma} c_{i \sigma}^{\dagger} c_{i \sigma}, \mu$ is the chemical potential, $\hat{t}=\left|t_{p s}\right|^{2} / \epsilon_{p}, V=V_{1} z\left|t_{s p \sigma} / \epsilon_{p}\right|^{2}$ and $U=U_{1}$. However, the validity of this reduction for realistic parameters and the relationship between $t, V$, and $U$ and $t_{s p \sigma}, V_{1}$, and $U_{1}$ clearly needs further study, for there are limits in which it must fail: in particular, it must fail in the limit when most of the $\mathrm{Bi}$ atoms are replaced by $\mathrm{Pb}$ atoms, so that $\mathrm{BaPbO}_{3}$ turns out to be metallic as it is. Nevertheless, this effective, one-band model, which has been proposed by a number of authors $110,18,78,79,80,82,83,84,85,86]$, can indeed serve as a starting point, at least in the semiconducting regime, as it contains, the effects of the electron-electron and electron phonon interactions (at the simplest level as discussed below).

In $\mathrm{BaBiO}_{3}, 1 / N \sum_{i \sigma}<\hat{n}_{i \sigma}>$ is constrained to be 1 ( $N$ is the number of lattice sites) corresponding to a nominal $\mathrm{Bi}$ valence of $4^{+}$. In the $\mathrm{Ba}_{1-x} \mathrm{~K}_{x} \mathrm{BiO}_{3}$ system, two important modifications are necessary:

(1) Since $\mathrm{K}^{1+}$ replaces $\mathrm{Ba}^{2+}$, one electron is removed from the system and $1 / N \sum_{i \sigma}<\hat{n}_{i \sigma}>=(1-x)$, corresponding to a nominal valence of $(4+x)^{+}$. (2) There is 
a random Coulomb potential because of $\mathrm{K}^{1+}$ sites, which act as effective $1^{-}$impurities.

In order to model 79 the $\mathrm{BaPb}_{1-x} \mathrm{Bi}_{x} \mathrm{O}_{3}$ system, we must introduce quenched, random, site variables $q_{j}=0$ (or 1 ), if site $j$ is occupied by a $\mathrm{Pb}$ (or $\mathrm{Bi}$ ) atom; i.e., $\bar{q}_{j}=x$, where the bar denotes an average over the quenched randomness. $U$ and $V$ in Eq.(II.2) are assumed to act only on Bi sites, which can be modelled by replacing $\hat{n}_{i}$ in Eq.(II.2) by $\hat{n}_{i} q_{i}$. In addition, we must introduce a random, site-energy term, $\epsilon_{0} \sum_{i \sigma} \hat{n}_{i \sigma}\left(1-q_{i}\right)$, with $\epsilon_{0}=2.5 \mathrm{eV}$, corresponding to the difference between the local orbital energies of $\mathrm{Pb}$ and $\mathrm{Bi}$. Note only Bi sites contribute electrons to the antibonding band, so $1 / N \sum_{i \sigma}<\hat{n}_{i \sigma}>=x$ (compare the case of doping above); however, $\mathrm{Pb}$ sites do not give rise to a Coulomb potential.

The justification for the negative- $U$ terms in Eqs. (II.1) and (II.2) is that it is the simplest phenomenological way of modelling the valence-skipping phenomenon, i.e., the stability of the $\mathrm{Bi}^{3+}\left(6 \mathrm{~s}^{2}\right)$ and $\mathrm{Bi}^{5+}\left(6 \mathrm{~s}^{0}\right)$ states relative to the $\mathrm{Bi}^{4+}\left(6 \mathrm{~s}^{1}\right)$ state, that many believe is characteristic of the chemistry of these bismuthates. As regards its origin, however, there are two points of view: the first phonon mediated and the other electronic.

The approach in which the negative $U$ arises from an electron-phonon mechanism is similar to Anderson's [87] suggestion (in a different context) that an attractive, electronelectron interaction can be generated if one couples lattice vibrations to electronic degrees of freedom strongly. Anderson had shown that the quenching of the magnetic moment in the A-15 compounds and some amorphous semiconductors can be explained if there is such an attractive interaction at each site. This idea was utilized by several authors to understand the barium bismuthates, as outlined below.

Rice and Sneddon [78 and later Jurczeck and Rice [79] used this idea and obtained the negative- $U$ model by coupling the excess charge $\left(\mathrm{Bi}^{4+}\right.$ being regarded as the neutral configuration) on a conventional positive- $U_{0}$ Bi site to the breathing-mode, optic phonon of the surrounding oxygen octahedron. Consider the $\mathrm{BaBiO}_{3}$ end of the composition 
range: The energy required to displace each $\mathrm{O}$ atom is

$$
H_{i j}=\frac{1}{2} C x^{2}-g x\left(q_{i}-q_{j}\right)
$$

where $q_{i}=\left(1-n_{i}\right)$ is the excess charge on a $\mathrm{Bi}$ site, $\left(q_{i}-q_{j}\right)$ is the charge difference between two neighbouring $\mathrm{Bi}$ sites, $x$ is the (breathing mode) displacement away from the equilibrium position of the oxygen atom, $g$ is the electron-phonon coupling constant, and $C$ is the spring constant of the oxygen breathing mode. The minimization of $H_{i j}$ (with respect to $x$ ) yields $x$ in terms $q_{i}$ and $q_{j}$ and thus eliminates the oxygen degrees of freedom. The complete Hamiltonian that now describes the conduction electrons in the Bi 6s band, including the long-range, Coulomb interactions between $\mathrm{Bi}$ sites is:

$$
H=\sum_{<i j>\sigma} t_{i j} c_{i \sigma}^{\dagger} c_{j \sigma}-g^{2} / 2 C \sum_{<i j>}\left(q_{i}-q_{j}\right)^{2}+\sum_{i \sigma} \frac{U_{0}}{2} \hat{n}_{i \sigma} \hat{n}_{i-\sigma}+\sum_{i \neq j}\left(e^{2} / \epsilon r_{i j}\right) q_{i} q_{j}
$$

The effective, onsite interaction $U_{\text {eff }}=U_{0}-z g^{2} / C$ is thus strongly suppressed compared to $U_{0}$, and can even be negative if $g$ is large enough ( $z$, the coordination number, is 6 in the three-dimensional cubic lattice considered here). Furthermore, $V_{n n}=\left(e^{2} / \epsilon r_{n n}+g^{2} / C\right)$ is the largest of the repulsive energies, where $r_{n n}$ is the nearestneighbour distance (lattice constant).

If we ignore the longer-range, repulsive interactions present in the Hamiltonian (II.3), we get an effective, one-band, negative- $U$, extended-Hubbard model involving Bi atoms only (Eq. (II.2)). We can incorporate the effects of the long-range part of the repulsive interactions roughly by replacing $e^{2} / \epsilon r_{n n}$ by $e^{2} \alpha / \epsilon r_{n n}$, where $\alpha$ is the Madelung constant. In any case $V$ in Eq. (II.2) is treated as a phenomenological constant, which must be obtained by fitting experimental data.

It has been suggested recently [18 that there is an electronic mechanism by which this attractive, onsite interaction can arise in the barium bismuthates. This mechanism 
is connected with the physics of valence skipping, electronic polarizability and screening processes. There are nearly 15 elements in the periodic table in Groups III and $V$ which have a propensity for skipping a valence state (Ga, In and $\mathrm{Tl}$ favour valence states $1^{+}$and $3^{+}$and not $2^{+}$; Bi and $\mathrm{Sb}$ favour $3^{+}$and $5^{+}$and skip $4^{+}$(Sec. I)). The intermediate valence states are either unstable or metastable. This was well known in chemistry and attributed to the favoured valence states corresponding to closed-shell electronic configurations: The effective, intra-atomic, repulsion energy $U_{n}$ for the $n^{\text {th }}$ charge-state of an ion, $U_{n} \equiv E_{n+1}+$ $E_{n-1}-2 E_{n}$, where $E_{n}$ is the total energy of the atom in the $n^{\text {th }}$ charge state, increases along a period for most elements as $n$ increases, since the sizes of the orbitals decrease with increasing $n$. However, for the valence skippers, it is clear from Table II.1 (compiled from measured or calculated ionization energies [18]) that $U_{n}$ has a minimum at the valence that is skipped. This reflects the larger correlation energy of the neighbouring, closed-shell configurations. All the $U$ values, including those at the skipped valence state, are still positive, as is necessary for an isolated ion. Varma [18] argues that this situation changes drastically when these ions are in a crystal. The value of $U$ that should then be used is the effective, onsite interaction after the screening effect of the environment has been accounted for. Consider $\mathrm{BaBiO}_{3}$ : The formal valence of $\mathrm{Bi}$ is $4^{+}$, so the configurations $\mathrm{Bi}^{5+}$ and $\mathrm{Bi}^{3+}$ are strongly charged. Also, each $\mathrm{Bi}$ atom is surrounded by the highly covalent and easily distortable $\mathrm{O}$ octahedra, thus nonlinear screening of the atomic charges is important. A negative value of $U$ (for $\mathrm{Bi}$ in the valence state $4^{+}$) results if the reductions in the energies of $5^{+}$and $3^{+}$configurations are sufficiently large compared to twice the reduction in the energy of the $4^{+}$configuration. Since the $6 \mathrm{~s}^{0}\left(\mathrm{Bi}^{5+}\right)$ configuration is most strongly charged compared to the $4+$ and $3+$ configurations, it is screened most strongly by charge transfer from the O octahedra to the 6p states of Bi. Density-functional calculations of Hamman (quoted in Ref. 18]) give $E\left(6 s^{0} 6 p^{1}\right)-E\left(6 s^{0}\right)=-43.3 \mathrm{eV}$. As shown in Table II.1, the electrostatic energy cost for the transfer of an electron is less than that obtained by linear interpolation between $U_{5+}$ and $U_{3+}$ by nearly $15 \mathrm{eV}$. Hence 
it is argued [18] that nonlinear screening can bring the effective $U_{4+}$ down to a negative value.

Thus both electron-phonon and electron-electron interactions can lead to phenomenological, effective Hamiltonians of the negative- $U$ type. However, these two mechanisms are very different when considered in detail. In particular, if the electron-phonon mechanism is involved, the effective, onsite, attraction $U$ is negative only on energy scales smaller than the frequencies of the breathing-mode phonons. If, on the other hand, an electronic mechanism is involved, $U$ can be negative over typical electronic energy scales (of order eV). This difference has detectable experimental consequences (Sec.IV).

Models (II.1)-(II.3) have strong correlation effects and many parameters, and are difficult to treat: Hence many workers [18, 80] have tried to see whether the large- $U$, limit $(U \gg z t)$ of model (II.2), which is much simpler (see below), is adequate for describing the properties of the $\mathrm{Ba}_{1-x} \mathrm{~K}_{x} \mathrm{BiO}_{3}$ system. In this limit we can project out the singly occupied $\left(\mathrm{Bi}^{4+}\right)$ states and keep only the empty $\left(\mid 0>\right.$ i.e., $\left.\mathrm{Bi}^{5+}\right)$ and doubly occupied $\left(\mid 2>\right.$ i.e., $\left.\mathrm{Bi}^{3+}\right)$ states at each site. The effective Hamiltonian in this reduced Hilbert space is easily derived via second-order, degenerate, perturbation theory (Appendix A) or canonical transformations, and can be written in terms of the pseudospin operators $\mathbf{S}_{\mathbf{i}}$ (that operate on the unoccupied and doubly occupied states $\mid i 0>$ and $\mid i 2>$, respectively, at the site $i)$ :

$$
\begin{gathered}
S_{i}^{+}=c_{i \uparrow}^{\dagger} c_{i \downarrow}^{\dagger} \equiv|i 2><i 0|, \\
S_{i}^{-} \equiv c_{i \downarrow} c_{i \uparrow} \equiv|i 0><i 2|, \\
S_{i}^{z} \equiv 1 / 2\left(n_{i}-1\right) \equiv(|i 2><i 2|-|i 0><i 0|) / 2 .
\end{gathered}
$$

The effective Hamiltonian is 


$$
\mathcal{H}_{e f f}=J \sum_{<i j>}\left(S_{i}^{+} S_{j}^{-}+H . C .\right)+K \sum_{<i j>} S_{i}^{z} S_{j}^{z}-B \sum_{i}\left(2 S_{i}^{z}+1\right)
$$

where $J=2 t^{2} / U, K=J+2 V$, and the pseudomagnetic field $B$ is related to the chemical potential $\mu$ via $B=\mu+U / 2-z V$. Note that in the experimental system $\mathrm{Ba}_{1-x} \mathrm{~K}_{x} \mathrm{BiO}_{3}$, $<S_{i}^{z}>$ is fixed at $-x / 2$; i.e., it corresponds to the fixed-magnetization ensemble.

An alternative representation, that is physically more transparent than the pseudospin representation, uses the hard-core boson operators $b_{i}^{+}=S_{i}^{+} \equiv c_{i \uparrow}^{\dagger} c_{i \downarrow}^{\dagger}$ and $b_{i}=S_{i}^{-} \equiv c_{i \downarrow} c_{i \uparrow}$. Clearly these represent real-space, onsite-paired $6 \mathrm{~s}^{2}$ electrons, or $\mathrm{Bi}^{3+}$ states, and have charge 2e. It is often useful to think in terms of these hard-core bosons, so we will use both pseudospin and hard-core-boson descriptions in our discussions below. The correspondences between the pseudospin model (II.5) and the extended-Hubbard model (II.2) are summarized in Table II.2. We will discuss the viability of these models mainly for $\mathrm{Ba}_{1-x} \mathrm{~K}_{x} \mathrm{BiO}_{3}$. To put our discussion in perspective, we review earlier theoretical treatments briefly.

\section{II.2 Brief Review of Earlier Theories of Barium Bismuthates}

\section{II.2.1 The Theory of Rice and Sneddon}

In one of the earliest attempts to understand the mechanism of pairing in the bismuthates, Rice and Sneddon [78, 79] developed the idea of the condensation of electrons, coupled strongly to the optic, breathing-mode phonons, into a crystal of real-space pairs (CDW order). These real-space pairs develop off-diagonal long-range order (ODLRO), if the band width is finite, and thence superconductivity.

Rice and Sneddon worked with an effective, classical spin model. They expressed the Hamiltonian (II.3), in the zero-bandwidth limit, in terms of a spin variable $m_{i}$ (where $\left.m_{i}=n_{i}-1\right)$ which assumes the values 1,0 , and -1 at each Bi site; $m_{i}=+1$ and $m_{i}=-1$ 
correspond, respectively, to the configurations with two electrons $\left(\mathrm{Bi}^{3+}\right)$ and no electron $\left(\mathrm{Bi}^{5+}\right)$ at the site $i$, respectively, whereas $m_{i}=0$ corresponds to single occupancy $\left(\mathrm{Bi}^{4+}\right)$ (the double degeneracy of this configuration is ignored). At the $\mathrm{BaBiO}_{3}$ end, the $\mathrm{Bi} 6 \mathrm{~s}$ band is half filled, so the chemical potential is $-U / 2$. The deviation of the chemical potential from its value at half filling $(=-U / 2)$ acts as a magnetic field in this effective spin model. Hence the effective, classical, spin Hamiltonian (in the zero-bandwidth limit) is just the $\mathrm{S}=1$, Blume-Emery-Griffiths (BEG) model [88,

$$
H=U_{e f f} / 2 \sum_{i} m_{i}^{2}+V \sum_{<i j>} m_{i} m_{j}
$$

where $U_{\text {eff }} / 2=\frac{1}{2}\left(U_{0}-z g^{2} / C\right), V=g^{2} / C+e^{2} \alpha / \epsilon r_{n n}$ (clearly $\left.V>0\right)$, and $\alpha$ is the Madelung constant (Sec.II.1). The intersite term is taken to be short ranged here and the effect of the longer-range part of the interaction is included via the Madelung constant. (Note the difference between the derivation of this classical spin model, obtained from model (II.2) by setting the hopping $t=0$ and ignoring the the double degeneracy of the $\mathrm{Bi}^{4+}$ configuration (up and down spin states), from our earlier derivation in Sec.II.1 of the quantum spin model in the limit of large- $U$, from a negative- $U$, extended-Hubbard model.)

The Hamiltonian (II.6) has been studied extensively [88 and its phase diagram is well known. For $U_{\text {eff }}<z V$ (always satisfied if $U_{\text {eff }}<0$ ), the ground state is antiferromagnetic, with $m_{i}= \pm 1$ on the two sublattices (i.e., the CDW phase of model (II.3)). If $U_{\text {eff }}>$ $z V$, the ground state has $m_{i}=0$ at all sites (i.e., a nonordered phase with all Bi sites equivalent).

Rice and Sneddon use mean-field theory to obtain the phase diagrams of model (II.3), with $t=0$, and the BEG model (II.6). Fig.II.1 depicts their phase diagrams in the $T-U_{\text {eff }} / 2 \mathrm{~V}$ plane. Though the mean-field phase boundaries obtained for these two models do not coincide, the topologies of the phase diagrams are the same. 
At finite temperatures there are two types of excitations from the CDW ground state: two-particle excitations $\left(m_{i} \rightarrow-m_{i}\right)$; and single-particle excitations $\left(m_{i}= \pm 1 \rightarrow 0\right)$, which break pairs. In the hypothetical limit $U_{\text {eff }} / 2 z V \rightarrow-\infty$, a transition from the CDW to the nonordered phase can occur only via the two-particle excitations; this is a second-order transition from a crystal of paired electrons to a liquid of these pairs. As $U_{\text {eff }} / 2 z V$ increases and passes through zero, the single-particle excitations become dominant and there is a first-order transition, with a discontinuous jump in the number of paired electrons (at $T=0$ this transition occurs at $U_{\text {eff }} / 2 z V=1$ ). For $U_{\text {eff }} / 2 z V>1$ no phase boundary is encountered, as $T$ increases; only the nature of the disordered phase changes from a liquid of paired electrons to a phase with no paired electrons.

Doping by $\mathrm{Pb}^{4+}$ is modelled as in dilute magnetic alloys since $\mathrm{Pb}^{4+}$ behaves like a $m_{i}=0$, nonmagnetic impurity. In this picture [78], the replacement of $\mathrm{Bi}$ by $\mathrm{Pb}$ removes an electronically active site from the system. Concentration fluctuations are assumed to be suppressed because of long-range Coulomb forces. At this level of modelling, the effect of doping by $\mathrm{Pb}$ is incorporated in the BEG Hamiltonian (II.6) by the reduction of the local coordination number z. For $U_{\text {eff }}<0$, both terms (site and bond)) in Eq. (II.6) favour real-space pairing, and there is a second-order transition to the nonordered state. However, if $U_{e f f}>0$, then these terms compete. The reduction of the average cordination number $\bar{z}$ decreases the importance of the bond term, so it is argued that dilution can drive the transition first order. Their phase diagram (Bethe-Peierls approximation) in the $T / V-\bar{z}$ plane is shown in Fig.II.2 for three different values of $U_{\text {eff }} / 2 V=-5,1.2$, and 2.3 .

The only ordered phase of the classical model (II.6) is the CDW phase. In order to have a superconducting phase, one must consider the quantum limit (with transverse spin fluctuations), so hopping (i.e., $t_{i j}$ ) must be included. With nonzero $t_{i j}$, it is not clear whether this model has an insulator-metal or an insulator-superconductor transition. Superconductivity may develop [78 via a transition from real-space, local pairing (a 
CDW) to momentum-space pairing (a BCS superconductor), aided by dilution (i.e., an increase of the effective, single-partcle band width); this scenario is described below:

In the $x \rightarrow 1$ limit (the $\mathrm{BaBiO}_{3}$ end), the CDW is the ground state, and two-particle excitations propagate only through high-energy intermediate states, resulting in a very narrow band. With dilution we get $\mathrm{Pb}^{4+}$ sites, which are $m_{i}=0$, local, single-particle excitations over the $\mathrm{CDW}$ ground state. As the concentration of $\mathrm{Pb}$ sites increases, these excitations can move because of the hopping $t_{i j}[=2(z-\bar{z}) t]$, so doping increases the band width for the motion of single-particle excitations.

In the $x \rightarrow 0$ limit (the $\mathrm{BaPbO}_{3}$ end), there are very few $\mathrm{Bi}$ atoms. They behave as impurities in $\mathrm{BaPbO}_{3}$. If $U_{\text {eff }}>0$ they prefer to be in the $4^{+}$state i.e., $m_{i}=0(\bar{z}=$ $z x \simeq 0$, so the bond term in Eq. (II.6) can be neglected) and act as donors of electrons. As $x$ and hence $\bar{z}$ increases, a metal-insulator transition becomes possible. If, however, $U_{\text {eff }}<0$, the Bi sites act as negative- $U$ centres and charge disproportionation into $\mathrm{Bi}^{3+}$ and $\mathrm{Bi}^{5+}$ is possible; i.e., an electron binds to the $\mathrm{Bi}^{4+}$ site because of the short-range, onsite, Coulomb potential. This cannot occur unless $\left|U_{e f f}\right| / 2 \gg 2(z-\bar{z}) t$. Hence Rice and Sneddon argue that [78], with decreasing $\bar{z}$ (i.e., increasing the $\mathrm{Pb}$ concentration) and at $T=0$, there can be a transition from a CDW insulator to a metal only if $\left|U_{\text {eff }}\right| / 2$ is not too large. If $t_{i j}$ increases relative to the electron-phonon interaction (in the conventional BCS theory, $t_{i j}$ is much stronger than the electron-phonon coupling), then the transition to an insulator from a metal occurs at a larger value of $\bar{z}$, (i.e., a higher Bi concentration is necessary to make the system insulating). If $t_{i j}=0$, each electron is localized at a site and, for a system with $N$ electrons, there are $2^{N}$ states. The spin degeneracy of the unpaired state is removed in the metallic state $\left(\right.$ when $\left.t_{i j} \neq 0\right)$. As a result the entropic correction to the free energy goes as $T^{2}$, in the metallic state, as opposed to $T$ in the unpaired state (with $t_{i j}=0$ ). Thus the metal-insulator phase boundary moves up perpendicular to the $T=0$ axis in the $T / V-\bar{z}$ phase diagram (Fig.II.2). At lower temperatures, 
then, the metal can become a supeconductor. This is the usual BCS (momentum-space) condensation of paired electrons into a superconducting state. Since the superconductivity here is mediated by optic phonons, the attractive interaction is retarded and all energy integrals (e.g., in the $T_{c}$ equation) are cut off by $\omega_{0}$, the frequency of the breathing-mode optic phonon.

\section{II.2.2 The Theory of Jurczek and Rice}

The transition from real-space to momentum-space pairing as a function of doping |78 described above was studied further [79 in the limit of finite and large bandwidth, to understand why the CDW phase in the lead-doped system is so stable (till a $\mathrm{Pb}$ concentration of $65 \%$ ). The mean-field study of a strongly coupled electron-phonon system (with parameters taken from band-structure calculations [3, 4]) showed that the "local" CDW state is indeed stable upto large doping levels. (Band-structure calculations (Secs. I and II.3.1) had predicted a strongly hybridized antibonding $\mathrm{Bi} / \mathrm{Pb} 6 \mathrm{~s}-\mathrm{O} 2 \mathrm{p}_{\sigma}$ band crossing the Fermi surface as in a metal, which was clearly untenable since the lead-doped system shows a metal-insulator transition at a $\mathrm{Pb}$ concentration of $65 \%$.) This theory begins with the Hamiltonian (II.3) and an electron-phonon coupling that is strong enough to make $U_{e f f}=U_{0}-z g^{2} / C$ negative, which can lead to both CDW and pairing instabilities. We outline this study below (first for the undoped and then for the doped case.)

\section{Undoped Case}

Here $x=1$ so the (uniform) CDW order parameter is:

$$
<\hat{n}_{i}>\equiv<\sum_{\sigma} c_{i \sigma}^{\dagger} c_{i \sigma}>=1+b_{c} \exp i \mathbf{Q} \cdot \mathbf{r}_{i}
$$

where $\mathbf{Q}=\pi(1,1,1)$ and $b_{c}$ measures the deviation of the valence at a $\mathrm{Bi}$ site from its nominal value $(4+)$. Bi sites with $<\hat{n}_{i}>=1+b_{c}$ and $<\hat{n}_{i}>=1-b_{c}$ are called $\mathrm{A}$ and $\mathrm{B}$ sites, respectively. With this choice for the order parameter and a conventional mean-field 
decoupling the Hamiltonian becomes

$$
H_{0}=t \sum_{<i j>\sigma} c_{i \sigma}^{\dagger} c_{j \sigma}+\Delta_{c} \sum_{i} \hat{n}_{i} \exp i \mathbf{Q} \cdot \mathbf{r}_{i}
$$

where $\Delta_{c}=u_{p} b_{c} / 2$ is the CDW gap parameter, $u_{p} \equiv U_{e f f}+2 z e^{2} \alpha / \epsilon r_{n n}$, and $\alpha$ is the Madelung constant. The charge-disproportionation response-function is

$$
R\left(b_{c}\right) \equiv 1 / N \sum_{j=1}^{N} \exp i \mathbf{Q} \cdot \mathbf{r}_{j} \int_{-\infty}^{E_{F}} d E n_{j}\left(E ; b_{c}\right),
$$

and, at half filling, the Fermi energy is given by

$$
1=1 / N \sum_{j} \int_{-\infty}^{E_{F}} d E n_{j}\left(E ; b_{c}\right),
$$

where $n_{j}\left(E ; b_{c}\right)$, the local density of states at the site $j$ with energy $E$, obviously depends on the value of $b_{c}$, which must be determined self consistently. If we multiply both sides of the self-consistency equation $<\hat{n}_{i}>=1+b_{c} \exp i \mathbf{Q} \cdot \mathbf{r}_{i}$ by $\exp i \mathbf{Q} \cdot \mathbf{r}_{j}$ and sum over all sites, it becomes $R\left(b_{c}\right)=b_{c}$. The local density of states (LDOS) that satisfies this selfconsistency equation can be obtained, as usual, from the imaginary part of the Green function:

$$
\begin{gathered}
n_{j}(E) \equiv<j\left|\delta\left(E-H_{0}\right)\right| j>=\frac{1}{\pi} \lim _{\eta \rightarrow 0} \operatorname{Im}<j\left|\frac{1}{E-i \eta-H_{0}}\right| j> \\
=\left.\frac{1}{\pi} \lim _{\eta \rightarrow 0} \operatorname{Im} G_{j}(z)\right|_{z=E-i \eta}
\end{gathered}
$$

Finally the self-consistency equation becomes the usual mean-field gap equation:

$$
1 / u_{p}=\frac{1}{2 N} \sum_{\mathbf{k}} 1 /\left(\Delta_{c}^{2}+t_{\mathbf{k}}^{2}\right)^{1 / 2}
$$


where the sum over momenta is restricted to half the Brillouin zone, $N$ is the number of sites in the lattice, $t_{\mathbf{k}}=t \sum_{\mathbf{k}} e^{i \mathbf{k} \cdot \mathbf{a}}$, and $\mathbf{a}$ are nearest-neighbour lattice vectors.

\section{Doped Case}

Jurczek and Rice accounted for dilution (i.e., Pb doping, Sec.II.1) by replacing $\hat{n}_{j}$ with $p_{j} \hat{n}_{j}$, where $p_{j}=1$, if site $j$ is occupied by a Bi atom, and $p_{j}=0$, otherwise; the difference $\epsilon_{0},(\simeq 2.5 \mathrm{eV}[3$, 4] between the onsite energies of $\mathrm{Pb}$ and $\mathrm{Bi}$, is an additional parameter. The following ansatz is used for the inhomogeneous, CDW order parameter:

$$
<\hat{n}_{j}>=p_{j}\left(1+b_{c} \exp i \mathbf{Q} \cdot \mathbf{r}_{j}\right)
$$

i.e., for a site with a $\mathrm{Pb}$ atom $<\hat{n}_{j}>=0$. Mean-field decoupling yields the Hamiltonian

$$
H_{0}=t \sum_{<i j>\sigma} c_{i \sigma}^{\dagger} c_{j \sigma}+\epsilon_{0} \sum_{j} \hat{n}_{j}\left(1-p_{j}\right)+\Delta_{c}(x) \sum_{j} \hat{n}_{j} p_{j} \exp i \mathbf{Q} \cdot \mathbf{r}_{j}
$$

where $\Delta_{c}(x)=\frac{1}{2}\left(U_{\text {eff }}+2 z e^{2} \alpha / \epsilon r_{n n}\right) b_{c}(x)$ and we have displayed the $x$ dependence of various quantities explicitly, as the order parameter has to be obtained self consistently for each value of $x$. For $\mathrm{BaBiO}_{3}$ there are two sets of inequivalent Bi sites, A and $\mathrm{B}$. With the introduction of $\mathrm{Pb}$, sites in each of these sets can have either a $\mathrm{Bi}$ or a $\mathrm{Pb}$ atom. Hence $R\left(b_{c}\right)$ (Eq. (II.8)) becomes

$$
\begin{aligned}
R\left(b_{c}(x)\right) & =\frac{1}{2 x(1-x)}\left[x \int_{-\infty}^{E_{F}} d E \overline{n_{A-B i}}\left(E ; b_{c}\right)+(1-x) \int_{-\infty}^{E_{F}} d E \overline{n_{A-P b}}\left(E ; b_{c}\right)\right. \\
& \left.-x \int_{-\infty}^{E_{F}} d E \overline{n_{B-B i}}\left(E ; b_{c}\right)-(1-x) \int_{-\infty}^{E_{F}} d E \overline{n_{B-B i}}\left(E ; b_{c}\right)\right] .
\end{aligned}
$$

The LDOS (Eq. (II.10)) must now be configurationally averaged. Even a coherentpotential-approximation (CPA) calculation of this averaged $\operatorname{LDOS}\left(\overline{n_{A-B i}}\right.$, etc.) is difficult since there are four different averages. Hence an orthogonalized-moment method has been 
used [79]. This method uses the continued-fraction representation of the one-particle Green function $G_{i}(z)$. Figures II.3a and II.3b show the self-consistent LDOS for the Bi$\mathrm{A}(\mathrm{B})$ sites at $\mathrm{Pb}$ concentrations of $20 \%$ and $60 \%$, respectively. Note that, on doping with Pb: (1) there is no real gap, but only a pseudogap, in the DOS at the Fermi energy; and (2) there are very few $\mathrm{Pb}$ states in the pseudogaps of Fig.II.3. As the $\mathrm{Pb}$ concentration increases, the Bi subbands approach each other: the upper Bi subband is pushed down because of repulsion by the $\mathrm{Pb}$ band (the difference between the onsite energies of $\mathrm{Pb}$ and $\mathrm{Bi}$ is $\left.\epsilon_{0}, \simeq 2.5 \mathrm{eV}\right)$ but the lower Bi band is hardly affected.

It was found [79] that the local CDW is not stable (i.e., $b_{c}=0$ ) if the total coupling constant $\lambda(x) \equiv \frac{1}{2}\left|U_{e f f}(x)\right| N\left(E_{F}, b_{c}=0\right)$ is less than a critical value $\lambda_{c}(x) .\left(N\left(E_{F}, b_{c}=0\right)\right.$ is the DOS at the Fermi energy in the absence of the CDW; and this positive, dimensionless, total coupling constant is an effective measure of the onsite attraction relative to the bandwidth in the noninteracting limit.) Also, the local CDW in the $\mathrm{BaPb}_{1-x} \mathrm{Bi}_{x} \mathrm{O}_{3}$ system was shown to be stable for a wide range $(0.35<x<1)$ with reasonable values of $\lambda$. In this local CDW phase the DOS is nonzero in the pseudo gap (Fig.II.3), so, to account for the semiconducting nature of this phase in $\mathrm{BaPb}_{1-x} \mathrm{Bi}_{x} \mathrm{O}_{3}$, Jurczek and Rice argued that the states in the pseudo gap obtained here could well be localized because of diagonal disorder (i.e., $\mathrm{Pb}$ doping).

An estimate of the electron-phonon coupling constant has also been made [79]: $U_{\text {eff }}$ can be separated into an attractive part $z g^{2} / C$, from the electron-phonon interaction, and $U_{0}$, the repulsive, onsite electron-electron Coulomb interaction. These two parts can be used to define the electron-phonon and electron-electron coupling constants $\lambda_{e p}$ and $\lambda_{e e}$, respectively ( $\lambda$ 's defined as above). When $U_{0}$ exceeds $U_{0 c}=z g^{2} / C$ the effective, onsite, interaction becomes repulsive and the ground state exhibits a spin-density wave, not a charge-density wave (if near-neighbour repulsion is negligible compared to the onsite term). Hence, in the CDW state, $\lambda_{e e}$ is maximum at $U_{0}=U_{0 c}$. This maximum 
value combined with typical values of $U_{0}$ and the value obtained for $\lambda_{c}$ gives an estimated lower bound for $\lambda_{e p}$ : it is found that $\lambda_{e p} \simeq 1$ (for $\epsilon_{0} \simeq 1.7 \mathrm{eV}$ ); i.e., according to this theory, the system is just in the strong electron-phonon-coupling regime. (This should be contrasted with the large values obtained in the bipolaron-superconductivity theories, discussed below.) The values of both $\lambda_{e p}$ and $\epsilon_{0}$ compare reasonably well with experimental observations: $\epsilon_{0} \simeq 2.5 \mathrm{eV}$ (atomic estimate given above) and the experimental value of $\lambda_{e p} \simeq 0.6$ in the lead-doped system. These values have been used [79] to obtain the superconducting $T_{c}$ via the McMillan-Morel-Anderson Formula (McMillan, (1968; Morel $\&$ Anderson, 1962) and with $\lambda_{e p}\left(b_{c}=0.65\right)=0.7: \quad T_{c}=\frac{<\omega>}{1.45} \exp \left(\frac{1.04\left(1+\lambda_{e p}\right)}{\lambda_{e p}-\mu^{*}\left(1+0.62 \lambda_{e p}\right)}\right)$. Here $\langle\omega\rangle$ is the average phonon frequency (this is normally taken to be the frequency of the phonon that couples to the electronic degree of freedom or the Debye temperature of the system), $\mu^{*}$ is the Morel-Anderson Coulomb pseudopotential (Morel \& Anderson, (1962). Jurczek and Rice obtained $T_{c} \geq 7 \mathrm{~K}$ with $<\omega>\simeq 150 \mathrm{~cm}^{-1}$, the frequency of the optic-mode phonon, and $0.067<\mu^{*}<0.116$.

The main results of the above analysis is that the insulating, local CDW phase of the lead-doped bismuthate is quite robust against doping. There is a strong electronphonon coupling in $\mathrm{BaPb}_{1-x} \mathrm{Bi}_{x} \mathrm{O}_{3}$ responsible for the attractive electron-electron interaction that leads to the CDW and superconducting instabilities in this system; and the electron-phonon coupling constant is fairly large. We will briefly discuss the merits and shortcomings of this model below. 


\section{II.2.3 CPA On An Attractive Hubbard Model: Semiconductor-Super- conductor Transition}

Yoshioka and Fukuyama [89] have considered a negative- $U$, Hubbard model with onsite disorder for the bismuthates. They believe that, in $\mathrm{BaPb}_{1-x} \mathrm{Bi}_{x} \mathrm{O}_{3}$, there is no semiconductor-metal transition but only a transition from a semiconductor to a superconductor (at $T=0$ ). Although such an assertion is not very unreasonable (Sec.I.3), there is no firm experimental evidence for it. In their model then, as long as there is a gap in the $\mathrm{DOS}$ due to the difference in chemical potentials of $\mathrm{Bi}$ and $\mathrm{Pb}$, the system remains insulating. As soon as this gap vanishes, the attractive onsite interaction leads to a pairing instability, which, in the doped case, leads to a superconducting transition (at half filling both the CDW and superconducting phases are degenerate, but doping favours superconductivity). We review their theory briefly below:

In the negative- $U$ Hubbard model they consider, disorder because of the substitution of $\mathrm{Bi}$ by $\mathrm{Pb}$ is modelled by a site-dependent chemical potential. Site energies $\epsilon_{A}$ and $\epsilon_{B}$ are associated with the atoms $\mathrm{A}$ and $\mathrm{B}$ (see below) respectively. There is an onsite, attractive interaction mediated by a strong, electron-phonon interaction. They further assume that atom $\mathrm{A}$ has no conduction electron while B has two (see below). So at $x=1$ the conduction band is full and at $x=0$, it is empty . Now the connection between

this picture of a binary alloy and the experimental system $\mathrm{BaPb}_{1-x} \mathrm{Bi}_{x} \mathrm{O}_{3}$ is made in the following manner:

The hybridization of $\mathrm{Bi} / \mathrm{Pb} 6 \mathrm{~s}$ and $\mathrm{O} 2 \mathrm{p}$ orbitals creates ten bands near the Fermi level (Sec.I, Fig.I.11 and I.13). The Fermi level falls in the highest $\sigma^{*}$ subband which is half-filled at $x=1$. A unit-cell-doubling distortion creates a gap along the entire noninteracting nested Fermi surface (Sec.I) crossing this subband. Hence this subband splits; and the lower split subbands is filled at $x=1$ and empty at $x=0$. Such a distortion corresponds to the frozen-in, breathing-mode phonon observed in this system. If the 
difference between $\mathrm{Bi}$ and $\mathrm{Pb}$ site energies were much smaller than this gap, then for discussing the low-energy physics of this system it would be sufficient to concentrate on the lower split subband only.

In the bismuthates, actually this is not the case; the $\mathrm{Pb}$ site energy is higher and the difference between atomic levels of $\mathrm{Pb}$ and $\mathrm{Bi}$ is $\simeq 2.5 \mathrm{eV}$ ) Then the mapping of the real system onto the alloy is done by assigning the $\mathrm{PbO}_{3}$ cluster to $\mathrm{A}$ atom and the $\mathrm{BiO}_{3}$ cluster to B atom (and ignoring the existence of the CDW for other purposes).

The resulting model is given by the Hamiltonian

$$
H=\sum_{<i j>\sigma}\left(t_{i j}+\frac{1}{2} \epsilon_{i} \delta_{i j}\right) c_{i \sigma}^{\dagger} c_{j \sigma}-\sum_{i \sigma} U \hat{n}_{i \sigma} \hat{n}_{i-\sigma},
$$

with $\epsilon_{i}=\epsilon_{A(B)}$ for $\mathrm{A}(\mathrm{B})$ atoms. The randomness is treated in the CPA, whereas the attractive interaction is treated via standard, mean-field approximation, it is i.e., assumed that $<\hat{n}_{i}>=n_{A(B)}$ for $\mathrm{i}$ belonging to $\mathrm{A}(\mathrm{B}) ; \mathrm{n}_{A}$ and $\mathrm{n}_{B}$ are determined self-consistently. In this approximation the mean-field Hamiltonian becomes

$$
H=\sum_{<i j>\sigma}\left(t_{i j}+\tilde{\epsilon}_{i \sigma} \delta_{i j}\right) c_{i \sigma}^{\dagger} c_{j \sigma}
$$

where $\tilde{\epsilon}_{i \sigma}=\frac{1}{2}\left(\epsilon_{i}-U<\hat{n}_{i-\sigma}>\right)$

To study the effect of disorder, we need $\varepsilon$, the difference between the chemical potentials of the $\mathrm{A}$ and $\mathrm{B}$ site in presence of the mean-fields, i.e.,

$$
\varepsilon=\varepsilon^{(0)}-U\left(n_{A}-n_{B}\right)
$$

where $\varepsilon^{(0)}=\epsilon_{A}-\epsilon_{B}$. The local densities of states at A and B sites are given in the CPA by 


$$
\rho_{A, B}=-\frac{1}{\pi} \operatorname{Im}\left(\frac{F(z)}{1-\left(\epsilon_{A, B}-\Sigma(z)\right) F(z)}\right)_{z=E+i 0},
$$

where the self energy calculated in CPA, is $\Sigma(z)=z-1 / F(z)-F(z) / 4$ and

$$
F(z)=1 / N \sum_{k} G\left(k, i \omega_{n}\right)
$$

Now $n_{A}$ and $n_{B}$ are determined self consistently via

$$
n_{A(B)}=\int_{-\infty}^{E_{F}} d E \rho_{A(B)}(E)
$$

and the lever rule $(1-x) n_{A}+x n_{B}=x$. Finally, assuming that there is a gap in the density of states in the semiconducting phase, we get (at $\mathrm{T}=0$ )

$$
n_{A}=\frac{x}{4} w^{2} / \varepsilon^{2} \text { and } n_{B}=1-\frac{1-x}{4} w^{2} / \varepsilon^{2}
$$

where, $w$ is half the noninteracting bandwidth, and thence

$$
\varepsilon=\varepsilon^{(0)}+U\left(1-\frac{w^{2}}{4 \varepsilon^{2}}\right)
$$

which gives the condition that $\varepsilon^{(0)}$ should satisfy to produce a gap in the density of states. Figures II.4a and II.4b show, respectively, the boundary between semiconducting and metallic phases and the DOS. The total DOS $\rho(E)$ (Fig.II.4b) is obtained from $\rho(E)=(1-x) \rho_{A}(E)+x \rho_{B}(E)$, where $x$ is determined self consistently from Eqs. (II.17) and the lever rule. With the DOS thus obtained, the superconducting $\mathrm{T}_{c}$ is calculated from a Cooper instability calculation in the framework of the CPA. The expression for $T_{c}$ is formally the same as for a pure system.

$$
1=\frac{U}{2} \int_{-\omega_{D}}^{\omega_{D}} d E \rho(E) \frac{\tanh \left[\left(E-E_{F}\right) / 2 T_{c}\right]}{\left(E-E_{F}\right)},
$$

while $\rho(E)$ is determined in the manner described above. 
Fig.II.5 shows $\mathrm{T}_{c}$ versus $x$ for different values of $U$. The cut off $\omega_{D}$ is some phonon frequency whose value is taken to be larger than the bandwidth in this calculation. but the phase diagram qualitatively the same. If the cut-off is reduced, $\mathrm{T}_{c}$ decreases. Hence depending on the parameter values of the system $\left(w, \varepsilon^{0}\right.$ and $\left.U\right)$ the model system becomes either a superconductor or a semiconductor as $x$ is varied. Note that the phase is symmetric about $x=0.5$.

The results obtained here do not really conform to the experimental observations in the $\mathrm{BaPb}_{1-x} \mathrm{Bi}_{x} \mathrm{O}_{3}$ system. The insulating phase here has no CDW order as observed in the system. This is a very simple-minded model only explore the possibility of a semiconductor-superconductor transition but it may not be relevant for the $\mathrm{BaPb}_{1-x} \mathrm{Bi}_{x} \mathrm{O}_{3}$ system.

\section{II.2.4 $\mathrm{Ba}_{1-x} \mathrm{~K}_{x} \mathrm{BiO}_{3}$ As A Doped Peierls-Insulator}

The models we have discussed so far are for the lead-doped bismuthate. They were all put forward before the discovery of superconductivity in $\mathrm{Ba}_{1-x} \mathrm{~K}_{x} \mathrm{BiO}_{3}$. Subsequent theoretical sork on this system has been hampered by the limited amount of experimental data that is available. We discuss below one of the few theoretical attempts 82 to understand the $\mathrm{Ba}_{1-x} \mathrm{~K}_{x} \mathrm{BiO}_{3}$ system. The objective of this study is limited to understanding the insulating behaviour in $\mathrm{BaBiO}_{3}$ system and its disappearence on doping with potassium.

Rice and Wang [82] interpret the $\mathrm{BaBiO}_{3}$ system as a Pierls insulator which undergoes a semiconductor-metal transition on doping because of the loss of the commensurate Peierls condensation energy. They assume that the ground state at $x=0$ is a bondordered, CDW rather than an onsite CDW i.e., a Peierls insulator in which there is an alternation of bond lengths. Experiments indicate the possibility of [26, 8, 29, 24] alternating $\mathrm{Bi}-\mathrm{O}$ bond length in $\mathrm{BaBiO}_{3}$ (though this bond-length alternation can equally well arise from a site diagonal CDW, as in the theories of Rice and Sneddon and Jurczek 
and Rice). While considering the effect of doping, and the consequent disappearence of the insulating, bond ordered phase, they consider only the $\mathrm{Ba}_{1-x} \mathrm{~K}_{x} \mathrm{BiO}_{3}$, as the lead-doped system is diagonally disordered and a consideration of disorder is absolutely essential for its description. On the other hand, in the $\mathrm{Ba}_{1-x} \mathrm{~K}_{x} \mathrm{BiO}_{3}$, potassium replaces barium and hence the effect of disorder is probably less important.

They start with the tight-binding model [3, 4] (\& Sec. I.3) involving the Bi 6s conduction band with $(1-x)$ electrons per unit cell in the $\mathrm{Ba}_{1-x} \mathrm{~K}_{x} \mathrm{BiO}_{3}$ system, hybridizing strongly with the three oxygen $2 \mathrm{p}_{\sigma}$ bands (with six electrons per unit cell ). The Hamiltonian they use is:

$$
H=\frac{\epsilon_{p}}{2} \sum_{j \sigma}\left(b_{j \sigma}^{\dagger} b_{j \sigma}-\frac{1}{2} \sum_{\delta} a_{j+\delta \sigma}^{\dagger} a_{j+\delta \sigma}\right)-\sum_{j \sigma \delta} V_{j, j+\delta}\left(a_{j+\delta \sigma}^{\dagger} b_{j \sigma}+H . c .\right)+C / 2 \sum_{j \delta} u_{j+\delta, j}^{2} .
$$

Here $b_{j \sigma}^{\dagger}$ creates an electron in a $\mathrm{Bi} 6 \mathrm{~s}$ orbital with energy $\epsilon_{p} / 2$ (the zero of energy is set midway between the energies of $\mathrm{Bi} 6 \mathrm{~s}$ and $\mathrm{O} 2 \mathrm{p}$ levels) at the site $\mathrm{j}$ of the reference cubic lattice (noncubic distortions are ignored); $a_{j \sigma}^{\dagger}$ is a similar operator for the $\mathrm{O} 2 \mathrm{p}_{\sigma}$ orbital (at energy $-\epsilon_{p} / 2$ ) and $j+\delta$ denotes one of the six nearest neighbour sites of $j$; $\mathrm{C}$ denotes the spring constant for the stretching of the $(j, j+\delta)$ band, described by $u_{j, j+\delta}$ the deviation from the equilibrium separation

$$
V_{j, j+\delta}=\operatorname{sign}(\delta)\left(V-\gamma_{c} u_{j, j+\delta}\right)
$$

is the hopping matrix element between $\mathrm{Bi}$ and $\mathrm{O}$ where $-\gamma_{c}$ is the derivative of $V_{j, j+\delta}$ with respect to $u$. The sign on the right hand side of Eq.(II.19) reflects the symmetry of the $\sigma$ bonds between $\mathrm{Bi} 6$ s and $\mathrm{O} 2 \mathrm{p}$. Here the phonon couples to the hopping of the electron, whereas in the model introduced by Rice and Sneddon and Jurczek and Rice (Sec.II.2.2) the phonon couples to the charge difference on neighbouring Bi sites. In the case $u_{j, j+\delta}=0$ for all $j$, the alloy is a metal and $\mathrm{H}$ is easily diagonalized to give two 
$\sigma$ bands and two non bonding dispersionless oxygen bands. In $\mathrm{BaBiO}_{3}$ (i.e., at $x=0$ ) the uppermost tight binding band (which is half filled) is unstable with respect to the commensurate Peierls distortion

$$
u_{i, i+\delta}=(-1)^{i} \Delta / \gamma_{c}, \text { for all } i
$$

Here $\Delta$ is the amplitude of the dimerization and has the dimensions of energy. This dimerization leads to a splitting of both the original $\sigma$ bands and we get four subbands with energies $E_{k, n=1 \cdots 4}$, given by

$$
\mathrm{E}_{k, n}= \pm\left[{\frac{\epsilon_{p}}{2}}^{2}+6\left(V^{2}+\Delta^{2}\right) \pm\left[(12 V \Delta)^{2}+\left(V^{2}-\Delta^{2}\right)^{2} \epsilon_{k}^{2} / V^{2}\right]\right]
$$

where $\epsilon_{k}=z V \sum_{\mathbf{a}} e^{i \mathbf{k} \cdot \mathbf{a}}$ a being the nearest-neighbour lattice vector.

The Peierls gap $E_{p}$ is the gap between the upper two bands (or, equivalently, the gap between the two lower bands) and

$$
E_{p}=\left[{\frac{\epsilon_{p}}{2}}^{2}+6(V+\Delta)^{2}\right]^{1 / 2}-\left[{\frac{\epsilon_{p}}{2}}^{2}+6(V-\Delta)^{2}\right]^{1 / 2} .
$$

At half filling, the three lower subbands are filled and the topmost subband (from the splitting of the $\sigma^{*}$ band) is empty. The total energy is then given by

$$
E_{t o t}=3 N C\left(\Delta / \gamma_{c}\right)^{2}+2 \sum_{k} E_{k, 1}
$$

The Peierls distortion parameter $\Delta$ is determined from the self-consistency condition obtained by setting $\partial E_{t o t} / \partial \Delta=0$. A second derivative of $E_{\text {tot }}$ with respect to $\Delta$ gives the restoring force for small displacements of $u_{j, j+\delta}$ from its equilibrium value (as obtained from the self-consistency equation).

According to this theory, then, the insulating gap of $\mathrm{BaBiO}_{3}$ has to be equal to this Peierls gap. So the Peierls gap is fitted to the optical gap $(2 \mathrm{eV})$ of $\mathrm{BaBiO}_{3}$ to obtain 
estimates for the parameters of this theory. Using $\epsilon_{p} / 2=-1.1 \mathrm{eV}$ and $V=2.2 \mathrm{eV}$ (values taken from the band-structure results of Mattheiss and Hamann [45], as discussed earlier) these authors estimate the $\mathrm{Bi}-\mathrm{O}$ spring constant $C=15.7 \mathrm{eV} / \mathrm{A}^{2}$ and the dimensionless electron-phonon coupling constant $\lambda_{e p}=2 \gamma_{c}^{2} 3 \pi C V=0.16$. With these choices they get the bond-alteration amplitude at $x=0$ to be $u \simeq 0.08 \AA$ which is quite close to the experimental value [6, 0, 30, 31] in $\mathrm{BaBiO}_{3}$.

Next, an estimate can be made for the critical concentration $x_{0}$ of potassium at which the Peierls distortion vanishes and hence a semiconductor-metal transition takes place via the vanishing of $\Delta$. This is easily done by partially emptying the second top-most (filled at $\mathrm{x}=0$ ) subband and self-consistently calculating the value of $\Delta . x_{0}$ turns out to be $11 \%$, considerably smaller than the experimental value of $x=0.40$ for the $\mathrm{Ba}_{1-x} \mathrm{~K}_{x} \mathrm{BiO}_{3}$ system.

\section{II.2.5 Local-Pair Superconductivity and Barium Bismuthates}

The local-pair model for superconductivity has been studied in detail by Micnas et. al 80 and several other people, mainly in the context of the possiblity of bipolaron superconductivity 85, 90] for a variety of systems (like $\mathrm{Ti}_{4} \mathrm{O}_{7}$ ) which show a CDW ordering. They studied the negative- $U$, extended-Hubbard model in the large $U$ limit. We do not review here the extensive literature on negative- $U$ models which are not directly related to the bismuthates.

The possible application of this local-pairing idea to the barium bismuthates has been pointed out by Varma [18]. He proposed that the barium bismuthates be modelled by a negative- $U$, extended-Hubbard model with strong onsite interaction $U$. In this limit, the model becomes identical to the local-pair model as mentioned above. He then noted that the phase diagram of the negative- $U$, extended-Hubbard model is qualitatively similar to that of the barium bismuthates. 
In the early local-pairing papers the negative- $U$ was attributed to the electron-phonon interaction (as in Rice and Sneddon); however, the local pairing and bipolaron mechanism requires an extraordinarily strong coupling between electrons and phonons to produce an adequately large and negative- $U$. Subsequently Varma has pointed out the possiblity of an electronic origin for the negative- $U$ and, in a recent article 80 on local pair superconductivity, this alternative has also been alluded to.

The Hamiltonian studied is the extended Hubbard Hamiltonian:

$$
H=t \sum_{<i j>\sigma} c_{i \sigma}^{\dagger} c_{j \sigma}-\frac{U}{2} \sum_{i \sigma} \hat{n}_{i \sigma} \hat{n}_{i-\sigma}+\frac{V}{2} \sum_{<i j>\sigma \sigma^{\prime}} \hat{n}_{i \sigma} \hat{n}_{j \sigma^{\prime}}
$$

In the local-pairing model one takes $U$ to be larger than the band width, so the negative- $U$, extended-Hubbard model (II.2) can be mapped onto the pseudospin model (II.5) and the phase diagram obtained via mean-field theory. Typical phase diagrams are qualitatively similar to those observed for the lead- and potassium-doped bismuthates. There are three distinct phases: (pseudospin) antiferromagnetic (CDW for model II.2), spin flopped (superconductor for (II.2)), and nonordered (metallic for model II.2). A first-order boundary separates the superconducting phase from the CDW phase; all other transitions are continuous. In the $T-x$ plane the first-order boundary should appear as a region of two-phase coexistence (although reported earlier [1], such a region has not been confirmed in later experiments with high-quality samples). The superconducting phase of this model consists of strongly bound, onsite-paired electrons. A condensation of these bosons gives rise to supeconductivity. The system is, of course, diamagnetic as the spin degree of freedom is suppressed by the attractive interaction. We will discuss this model later in Section III and show that superconductivity and a host of other phenomena in the barium bismuthates cannot be described in terms of local pairing.

\section{II.3 Other Related Work}


There have been some other recent attempts to understand the superconducting phase and high superconducting $\mathrm{T}_{c}$ in the barium bismuthates. An attractive interaction between doped carriers in a CDW background can be generated, in certain circumstances, by the exchange of CDW amplitude modes [91]. The local depression of the CDW amplitude because of doping leads to an attractive interaction for the doped carriers and mediates pairing in $k$-space. The superconducting transition is of the BCS type, with the phonon cutoff frequency $\omega_{D}$ replaced by a much higher scale of energy (the frequency of the CDW amplitude mode). The formalism is analogous to its positive- $U$ counterpart, namely, the spin-bag mechanism [124] for high- $\mathrm{T}_{c}$ Cuprates.

Some recent work [92] has tried to understand the semiconducting behaviour of the $\mathrm{Ba}_{1-x} \mathrm{~K}_{x} \mathrm{BiO}_{3}$ system starting from the model of Jurczek and Rice (Jurczek and Rice [79], see Sec.II.2.2). Their motivation was to estimate the various parameters of this model from the known experimental results on $\mathrm{Ba}_{1-x} \mathrm{~K}_{x} \mathrm{BiO}_{3}$.

They use the Jurczek-Rice Hamiltonian (II.3) and assume that this system is in a CDW ordered state in its semiconducting phase. There are two types of sites in the system: $\mathrm{Bi}^{3+}$ and $\mathrm{Bi}^{5+}$ at half filling with concentrations $c_{3+}=c_{5+}=0.5$ each. Doping by potassium changes them to $(1-x) / 2$ and $(1+x) / 2$, respectively. In this picture the origin of the semiconducting phase is quite simple: The $\mathrm{Bi}^{3+}$ and $\mathrm{Bi}^{5+}$ sites give rise to the lower and upper bands containing $2 c_{3+}$ and $2 c_{5+}$ states and if there is a gap between them, then the Fermi level is in the gap as the filling of the lower band is $2 c_{3+}=1-x$. The system is then an insulator. Manh,, et al. calculate the band structure of the system described above where they account for the disorder due to potassium doping in the usual Bethe-Peierls approximation and the interaction is treated in the mean-field approximation.

The local density of states is calculated by these authors in the alloy approximation: They use a continued fraction representation for one-particle Green function with three unknown parameters in the theory: onsite attraction $U$, nearest-neighbour Coulomb re- 
pulsion $V$, and the effective electron-phonon coupling constant $\lambda_{e p}\left(=g N\left(E_{F}\right)\right.$, where $g$ is the electron-phonon coupling strength (Eq. (II.3)) and $N\left(E_{F}\right)$ is the DOS at the Fermi level. All the energies are scaled by the band width (the value used by the authors is the width of the $\sigma$-antibonding band in $\mathrm{Ba}_{1-x} \mathrm{~K}_{x} \mathrm{BiO}_{3}$, which is nearly $6 \mathrm{eV}$ from band structure calculations). These authors try to fit the experimental values of the optical gap $E_{G}$ at $x=0$ where $E_{G}=2 \mathrm{eV}$ and at $x=0.4$ where, $E_{G}=0$, (i.e., where $\mathrm{Ba}_{1-x} \mathrm{~K}_{x} \mathrm{BiO}_{3}$ becomes metallic). With the gap in the DOS obtained by them at those values of $x$, they conclude that for $-3 e V<U<-2 e V$, and $\lambda_{e p}<0.7$ produce a good fit for the optical gap.

All the theoretical studies discussed so far are based on models where the onsite, Coulomb interaction is either suppressed (Rice-Sneddon case), or the onsite $U_{\text {eff }}$ is either very small or even negative and attractive, and the nearest-neighbour Coulomb repulsion produces a CDW state. There is, however, a suggestion [93] that these systems are positive- $U$ systems and their insulating phase is be a spin-Peierls insulator, melting at a high temperature into a resonating-valence bond liquid: One starts with a strongly coupled spin and lattice system plus a Heisenberg-Hubbard model for the spin and charge degrees of freedom. Now such a spin-Peierls state involving spins at each $\mathrm{Bi}^{4+}$ ion will lead to a modulation in the $\mathrm{Bi}-\mathrm{Bi}$ distance. As far as we know there is no indication of an alternating $\mathrm{Bi}-\mathrm{Bi}$ distance in the structural work on these systems. We do not present a detailed discussion of this model, in this review.

There is some work by Jin et al. [94] where a molecular dynamics simulation of phonon density of states is used to study the superconductivity in barium postassium bismuthate within a strong coupling Eliashberg formalism. These authors used a moderate electronphonon coupling strength $(\lambda \simeq 1)$ and assumed that strongth electron-phonon coupling exists even at high phonon energies $(30-60 \mathrm{meV})$. The $T_{c}$ that they could reach are fairly high. 


\section{II.4 Discussion}

Most of the theoretical work on the bismuthates starts with: the negative- $U$, extended Hubbard model to account for the experimental observation [6, 7, 19] of a CDW phase close to half filling and the occurrence of superconductivity on doping. The diamagnetic behaviour of these systems can also be explained by using this model since the attractive interaction suppresses the free-electron moments.

The theory of Sneddon and Rice was aimed at motivating the attractive $U$ model for the barium bismuthate system. They did not try to obtain experimental fits from this model: A qualitative description of the pairing was their objective. In the model of Jurczek and Rice the more realistic case of finite band-width and disorder was discussed. The important result obtained from this work is that the CDW can survive rather large doping. The $\mathrm{T}_{c}$ obtained from this model was low for realistic electronic DOS at $E_{F}$. Their model produces a pseudo-gap at the Fermi surface, instead of a real gap as seen in the experiments, even in the insulating side and does not account for the observed transport properties in the insulating regime.

The model of Yoshioka and Fukuyama focusses attention on the insulator-superconductor transition; The connection between their "alloy" and the real $\mathrm{BaPb}_{1-x} \mathrm{Bi}_{x} \mathrm{O}_{3}$ system is rather far-fetched. The phase diagram obtained by them is symmetrical about $x$ and $1-x$, which is not the case for the real system. The theory of metal insulator transition in potassium doped system put forth by Rice and Wang [82], although interesting, is not quite satisfactory as it does not consider the effect of Coulomb interactions. Also the

eventual development of superconductivity at low temperatures in the metallic regime of these systems is completely ignored. They obtaine a semiconductor-metal transition at a very low value of $K$ concentration $(11 \%)$ compared to the experimental value $(\simeq 40 \%)$.

The local pairing mechanism for the superconductivity in these systems essentially 
involving the large $U$ limit of the negative $U$, extended Hubbard model, can not produce reliable experimental fits of both the superconducting and the normal properties. It produces too small a superconducting coherence length and too large an optical gap. The theory of charge-bag can produce a superconducting $\mathrm{T}_{c}$ that is large but does not account for the observed unusual transport properties in the CDW or semiconducting phases of these systems. The positive $U$ model has not so far been used to calculate the normal and superconducting properties.

The unusual experimental observation of the wide separation between the optical and transport gaps [2] has not been addressed by any of the theories we discussed. We find that this two-gap feature, coupled with other experimental observations, both in the superconducting and the normal phase of the bismuthates, puts quite stringent constraints on the models for these systems. We have recently explained this experimental observation and developed a theory for the bismuthate systems [10, 11] which can explain many of the experimental observations in these systems on the basis of the negative- $U$, extendedHubbard model; we discuss this in the following sections.

The negative- $U$ generated by an electron-phonon coupling is retarded (and hence cut off in energy), whereas the negative- $U$ generated by electronic processes acts over the entire non-interacting band-width. This makes the two mechanisms quite different, with different experimental manifestations. This is another major issue we focus on We argue that the two well-separated gaps can be understood only if the attraction is electronic in origin and suggest experiments to verify this assertions. We also calculate various normalstate and superconducting properties from the negative- $U$ extended Hubbard model and the values of the parameters of this model that best fit experimental data.

Though there are strong phenomenological grounds (Sec. II.1) for using the negative- $U$ extended Hubbard model for the bismuthates, there is no clear derivation of the negative$U$. In our work, it is used as a parameter of the theory. In Sec.III we discuss the large- $U$ 
limit of this model where a mapping onto a pseudospin, antiferromagnetic, anisotropic Heisenberg model (II.5) allows us to investigate the spin model. We discuss briefly the mean-field theory for this model and obtain various correlation functions in the random phase approximation (RPA). We also suggest a novel mechanism of charge transport via pseudo-spin exchange. We discuss the limitations of the large $-U$ limit and motivate the more interesting and appropriate limit of intermediate $U$ which we take up in Section IV.

\section{The Negative- $U$, Extended-Hubbard Model: Large- $U$ Limit}

\section{III.1: Summary of Results}

In Sec.II we motivated the use of the negative- $U$, extended-Hubbard model (II.2) for the barium bismuthates. It has been suggested [18] that, for a preliminary understanding, these bismuthates be modelled by the simpler Hamiltonian (II.5), since theoretical studies of this model are far easier than those of model (II.2). Model (II.5), an anisotropic, pseudospin $S=1 / 2$, Heisenberg antiferromagnet in a pseudomagnetic field, is the large- $U$ limit $(U \gg z t)$ of the negative- $U$ (i.e., onsite attraction) Hubbard model (II.2) with nearest-neighbour repulsion $V$. (We follow the convention that $U$ and $V$ are always positive; the sign of the interaction is displayed when needed.) It has been studied via different approximations by many workers [9, 10, 18, 79, 80]. Here we examine the viability of this limit for the barium bismuthates by doing detailed calculations for the phase diagram of this model and the properties - thermodynamic functions, correlation functions, and response functions - of its metallic, semiconducting, and superconducting phases [9, 10] and comparing them with experimental observations. Our overall conclusions are: (a) On the positive side, even the anisotropic-Heisenberg limit of the extended-Hubbard model leads to various results which are either in qualitative accord with experiments or which 
suggest new experiments for the elucidation of the properties of these alloys. (b) However, it is not good enough to obtain many important properties of these systems; its most serious limitation is that it leads to coherence lengths in the superconducting phase that are far too small compared to those obtained experimentally $(\simeq 70-80 A)$. We are thus led to use the intermediate- $U$ limit of model (II.2), which we use in Sec.IV to obtain better agreement with experiments. We summarize our large- $U$ results below:

(1) In the simplest, mean-field approximation, the anisotropic, $S=1 / 2$, Heisenberg antiferromagnet exhibits, typically, three phases, which, in the language of the extendedHubbard model, are (seeSec.III for the correspondences between the two models): a nonordered metal (NO), a charge-ordered semiconductor (with a CDW), and an swave superconductor (SC). Phase transitions between all excepting superconducting and CDW phases are continuous (second order); the superconductor-CDW transition is first order. Slight perturbations of the model (say by the inclusion of nextnearest-neighbour couplings) can yield an intermediate phase that exhibits both superconducting and CDW ordering 95. At special values of coupling constants, bicritical, tetracritical, and tricritical points or critical end points can be obtained.

(2) A random-phase-approximation (RPA) calculation of the excitation spectra in these phases shows the existence of a gap $(\simeq V)$ in their semiconducting, CDW phase, which is different from the gap $(\simeq U)$ for the breaking of pairs in the superconducting phase, which should appear in the optical spectrum.

(3) It is useful to think of the phases in the following way: $\mathrm{Bi}^{3+}\left(6 \mathrm{~s}^{2}\right.$ configuration) corresponds to a real-space pair of two electrons and constitutes a charge-2e, hardcore boson. The CDW phase is a crystal of these bosons, the superconducting phase a superfluid resulting from their bose condensation, and the nonordered-metallic phase their normal-liquid phase. 
(4) Charge conduction occurs by the motion of these charged bosons, or equivalently, by the exchange of pairs of $\mathrm{Bi}^{3+}$ and $\mathrm{Bi}^{5+}$ states. For this conduction mechanism the current operator has been constructed [9] and thence, using the RPA, the frequencydependent conductivity $\sigma(\omega)$ can be obtained [10]. It is shown that:

(a) In the nonordered-metallic phase the conductivity $\sigma(\omega)$ has a Drude form, i.e., $\sigma(\omega)=$ $n(2 e)^{2} \tau / m^{*}(1-i \omega \tau)$, with $\tau$ a phenomenological transport lifetime that accounts for scattering, $m^{*}$ the effective mass, and $n$ the number of paired carriers; in the grand-canonical ensemble, i.e., with fixed chemical potential, goes to zero exponentially as the temperature goes to zero; with fixed (i.e., at fixed filling), this means that the metallic phase does not extend down to zero temperature at any filling (See Sec.IV).

(b) In the CDW semiconducting phase the conductivity shows an activated behaviour, i.e., $\sigma(\omega \rightarrow 0) \simeq \exp \left(-\Delta / K_{B} T\right)$, where $\Delta$ is the gap (measured from the chemical potential) in the spectrum of the hard-core bosons ( or, equivalently, the pseudospinwave excitation spectrum ) in the CDW phase; this is in accord with experiments.

(5) Exactly as in the case of the bismuthates, the CDW phase of the model exhibits two gaps - a charge gap associated with the breaking of $\mathrm{Bi}^{3+}$ pairs, which costs an energy $\simeq U$ and another ( $\Delta \simeq V$, the nearest-neighbour repulsion) associated with the activated behaviour of $\sigma(\omega \rightarrow 0)$. We calculated [9] the optical conductivity in the single- and two-particle channels using the Kubo formula and the RPA in the large- $U$ limit. The result is shown in Fig.III.4. It is interesting to note that even at a simple level of approximation [10], the peak in the two-particle channel (at 0.24 $\mathrm{eV}$ ) is down by a factor of 9 compared to that in the one-particle channel (at $2 \mathrm{eV}$ ).

(6) The metallic phase of this model is not a conventional Fermi liquid, but rather a charged, hard-core, bose liquid, and must have exotic properties. For example, the 
specific heat is certainly not a linear function of the temperature T. (A crossover to a conventional Fermi liquid occurs as $U$ decreases.)

We now show explicitly the calculations that lead to the above results. The calculations are described in two parts: The first part (III.2.1) is devoted mainly to to the mean-field theory of the anisotropic, pseudospin Heisenberg model (II.5). In the following section (III.2.2) we show the calculations that lead to the excitation spectra in RPA in each of these mean field phases. We use the RPA excitation spectra then to obtain various correlation functions. We discuss the novel conductivity mechanism that we suggest for the bismuthates in their insulating phase and calculate the corresponding current operator in the large $-U$ limit.

\section{III.2 Calculations: An Outline}

\section{III.2.1 Mean-Field Phase Diagram}

The phase diagram of the pseudospin model (II.5) is well known at the level of the mean-field approximation; however, some recent treatments are misleading [80, 86], so we outline briefly how these phase diagrams are obtained [9].

The order parameters chosen are $\left\langle S_{i}^{x}>\right.$ and $\left\langle S_{i}^{z}>\right.$ where $i$ is the site index and can have only two labels A and B (indicating the two sublattices ). Also the rotational symmetry in pseudospin space about the $z$-axis (the magnetic field picks out the $z$ direc-

tion) allows one to choose the spin to be in the $x z$ plane, i.e., $\left\langle S_{i}^{y}\right\rangle=0$, without any loss of generality. A straightforward mean-field decoupling scheme is used to reduce the Hamiltonian (II.5) to the following site-diagonal mean-field Hamiltonian:

$$
\mathcal{H}_{m f}=J \sum_{<i j>} S_{i}^{x}<S_{j}^{x}>+\sum_{<i j>} S_{i}^{z}\left(K<S_{j}^{z}>-B\right)
$$


This Hamiltonian is diagonalized by the following eigenfunctions

$$
\left|i+>=\cos \frac{\theta_{i}}{2}\right| \uparrow>+\sin \frac{\theta_{i}}{2} \mid \downarrow>
$$

and

$$
\left|i->=\cos \frac{\theta_{i}}{2}\right| \downarrow>-\sin \frac{\theta_{i}}{2} \mid \uparrow>
$$

where

$$
\tan \theta_{i}=J_{0}<S_{j}^{x}>/\left(B-K_{0}<S_{j}^{z}>\right)
$$

and $\mid \uparrow>$ and $\mid \downarrow>$ are the eigenvetors of $S^{z}$ operator with eigenvalues $+1 / 2$ and $-1 / 2$, respectively. Here $J_{0}=z J$ and $K_{0}=z K$ and $\beta=\left(K_{B} T\right)^{-1}$. The corresponding eigenvalues are

$$
\Lambda_{i}^{ \pm}=-B \mp R_{j}
$$

with

$$
R_{j}^{2}=\left(J_{0}<S_{j}^{x}>\right)^{2}+\left(B-K_{0}<S_{j}^{z}>\right)^{2} .
$$

The mean-field free energy per site is

$$
\begin{aligned}
& f=-\frac{1}{\beta}\left[\log \left(2 \cosh \left(\beta R_{A}\right)\right)+\log \left(2 \cosh \left(\beta R_{B}\right)\right)\right] \\
& +J_{0}<S_{A}^{x}><S_{B}^{x}>-K_{0}<S_{A}^{z}><S_{B}^{z}>-B .
\end{aligned}
$$

(This can also be obtained by using Peierls or Gibbs-Bogoliubov variational method. 97] Finally the self-consistency equations follow easily by minimising the free energy with respect to the order parameters:

$$
\begin{gathered}
<S_{i}^{x}>=\left(J_{0}<S_{j}^{x}>/ 2 R_{j}\right) \tanh \left(\beta R_{j}\right) . \\
<S_{i}^{z}>=\left[\left(B-K_{0}<S_{j}^{z}>\right) / 2 R_{j}\right] \tanh \left(\beta R_{j}\right) ;
\end{gathered}
$$


The subscripts $i$ and $j$ label nearest-neighbour sites; i.e., they belong to the two different sublattices $\mathrm{A}$ and $\mathrm{B}$ (or $\mathrm{B}$ and $\mathrm{A}$ ), respectively. If more than one solution is obtained for these self-consistency equations, then the one which yields the lowest value of the free energy is picked.

Note that we can work with either fixed pseudomagnetic field (the analogue of the grand-canonical or fixed-chemical-potential ensemble for the original, extended-Hubbard model) or fixed $\left\langle S^{z}>\right.$ (the analogue of the canonical or fixed-filling ensemble); the results obtained from both approaches have to be the same by virtue of the equivalence of the two ensembles (see Note 2).

The phase diagram in both canonical and grand-canonical ensembles have been obtained. A typical phase diagram that results from this mean-field calculation is shown, in Fig.III.1a, in the $B-T$ plane and, in Fig.III.1b, in the $<S^{z}>-T$ plane. (Parameters for this phase diagram are: $J_{0}=1, K_{0}=1.301$.) There are three phases: a nonordered metal at large $B$ or $T$ (the pseudospin-model analogue is a paramagnet with some alignment of the pseudospins by virtue of the finite magnetic field), a CDW semiconductor (the pseudospin-model analogue: a ferrimagnet (antiferromagnet at $B=0)$ ), and an s-wave superconductor (pseudospin-model analog: a spin-flopped phase, with nonzero $x$ component of pseudospins). The pseudospin-model analogues can be obtained easily by consulting Table II.2. In terms of the hard-core bosons introduced above, these three phases correspond, respectively, to a bose liquid, a bose crystal, and a bose-condensed superfluid (note 3) phase.

Full lines in Fig.III.1a indicate continuous, second-order transitions, whereas the dashed line in Fig.III.1a indicates a first-order phase boundary; this boundary shows up as a region of two-phase coexistence if $\left\langle S^{z}\right\rangle$ (i.e., the filling) is held fixed (Fig.III.1b). If the temperature and filling are such that the system lies in this two-phase region, then, in equilibrium, the model system phase separates into two regions - one superconduct- 
ing and the other semiconducting - with one interface between them. This two-phase region is not a new mixed phase with both superconducting and CDW order. To obtain such a phase, next-nearest-neighbour interactions are required in the pseudospin model (II.5) (see below). Note that at no value of the filling does the metallic phase extend down to $T=0$. (This manifests itself in an exponential depletion of the carrier density if one tries to approach $T=0$ at fixed B.) The two second-order lines meet the first-order boundary (Fig.III.1a) at a bicritical point. The positions of these second-order lines can be obtained analytically as described in Appendix B. As Varma has noted 18] in this pseudospin-model limit the maximum $T_{c}$ for the superconducting phase occurs at the value of filling for which the metal-semiconductor transition first appears, i.e., at the filling at which the bicritical point occurs. (Strictly speaking, this maximum occurs at a slightly higher temperature. The two critical lines come in tangentially at the bicritical point, but this becomes evident only from a renormalization-group calculation.)

It is well known from the work of Liu and Fisher [95] and Matsuda and Tsuneto [96] that slight modifications of the pseudospin model (II.5) can lead to more complicated phase diagrams, at least at the level of mean-field theory. In particular, if we allow for next-nearest-neighbour interactions of the form $\sum_{<<i j\rangle>} J_{i j}^{\prime} S_{i} . S_{j}$, where $<<i j>>$ indicates that $i$ and $j$ are next-nearest neighbours, then, depending on the relative sizes of the different interactions in the model, phase diagrams of the sort shown schematically in Fig.III.2 can be obtained. (Such next-nearest-neighbour interactions could arise from next-nearest-neighbour repulsion $V^{\prime}$ or hopping $t^{\prime}$ in the original extended-Hubbard model.) With such couplings it is possible to get a bona fide intermediate phase in which both CDW and superconducting order are present (Fig.III.2). As in Fig.III.1 solid lines denote continuous transitions and dashed ones first-order transitions. Note that a variety of multicritical points can be obtained now 998, 99]: bicritical, tricritical, and tetracritical points, and also a critical end point. We mention these multicritical points here and discuss them briefly in Appendix D mainly for the sake of completeness. It is quite likely 
of course that the precise multicritical behavior that obtains in the barium bismuthates depends sensitively on effects that are not included in our model, such as disorder, longrange Coulomb interactions and deviations from a cubic crystal structure, etc. Also, it will not be very easy to determine the nature of such multicritical behavior experimentally, for that will require very accurate control of both the temperature and the concentration of the dopant.

The phase boundary between the CDW and nonordered metallic phases belongs to the three-dimensional Ising universality class and the superconductor-nonordered metal transition to the three-dimensional $X Y$ class. The transitions from the intermediate phase (Fig.III.2) to the CDW and superconductor phases are, respectively, in the threedimensional $X Y$ and Ising universality classes. In the pseudospin model, the coherence length in the superconducting phase is very small (see Appendix F), so apparent meanfield exponents will not be seen here as they might well be in the barium bismuthates, in which coherence lengths are of the order of $80 \AA$.

\section{III.2.2 Excitation spectra, Correlation function and the Optical Conductivity in the Random-Phase Approximation}

The excitation spectra in all the phases of the pseudospin model can be obtained in a straightforward manner by using the equation-of-motion method followed by the random-phase approximation [100] as shown in Appendix C.

We use the Hubbard operators [100] $\hat{P}_{\alpha \beta}^{i} \equiv|i \alpha><i \beta|$, where the state $\mid i \alpha>$ refers to the states $\mid+>$ or $\mid->$ at the site $i$, in the mean-field Hamiltonian described above. The double-time retarded Green functions for these operators are denoted by $G_{\alpha \beta, \gamma \delta}^{m, n}\left(t-t^{\prime}\right)$ or, equivalently, $<<\hat{P}_{\alpha \beta}^{m}(t) \mid \hat{P}_{\gamma \delta}^{n}\left(t^{\prime}\right)>>$. The Green function is defined in the standard way as 


$$
G_{\alpha \beta, \gamma \delta}^{m, n}\left(t-t^{\prime}\right)=-i \theta\left(t-t^{\prime}\right)<\left[\hat{P}_{\alpha \beta}^{m}(t), \hat{P}_{\gamma \delta}^{n}\left(t^{\prime}\right)\right]_{-}>
$$

where $[\mathrm{P}, \mathrm{Q}]_{-}$denotes a commutator between the operators $\mathrm{P}$ and $\mathrm{Q}$.

The equations of motion for the Green functions are easily obtained in the randomphase approximation [9, 80]. The poles of these Green functions can be obtained simply from the solutions to the equations of motions. From these poles we can obtain the excitation spectra in the various phases of Fig.III.1. The details are discussed in the Appendix C.

In Fig.III.3 we show the pseudospin-wave excitation spectra in the three phases (respectively, CDW, superconductor, and nonordered metal) of the phase diagram of Fig.III.1a. The spectra are drawn along the symmetry directions of a cubic Brillouinzone. Data for these spectra are given in the figure captions. Note that there is a gap as expected in the CDW (i.e., ferrimagnetic in the pseudospin language) phase. This gap is obtained from the dispersion relation in the CDW phase:

$$
\omega^{2}=2 B-K_{0} m \pm\left[K_{0}^{2} \eta^{2}+\left(m^{2}-\eta^{2}\right) J^{2}(\mathbf{q})\right]
$$

Here $\eta=<S_{i}^{z}>-<S_{j}^{z}>$ is the staggered magnetisation. The \pm sign indicates that there are two branches in the spectrum, as should be the case in an antiferromagnetically ordered spin system. There is a gap in the spectrum of the non-ordered metallic phase also at any finite magnetic field which is merely the chemical-potential gap of the charged bose liquid. (In the pseudospin language this is the gap due to the aligning pseudomagnetic field.) From this expression it is easy to see that the gap at $T=0$ in the CDW phase is

$$
\Delta=\left[K_{0}^{2}-J^{2}(0)\right]^{1 / 2}
$$

Where 


$$
\begin{gathered}
J(\mathbf{q})=J_{0} \gamma_{\mathbf{q}}, \\
\gamma_{\mathbf{q}}=\frac{1}{z} \sum_{\mathbf{a}} \exp (i \mathbf{q} \cdot \mathbf{a}),
\end{gathered}
$$

with $\{\mathbf{a}\}$ is any one of the nearest-neighbour lattice vectors,

and

$$
m \equiv n-1=<S_{i}^{z}>+<S_{j}^{z}>
$$

The gap $\Delta$ vanishes (as does the CDW phase) as the anisotropy goes to zero, i.e., as $\mathbf{K}$ starts to become equal to $\mathbf{J}$. This is the transport gap $E_{A}$ that shows up in measurements of the dc conductivity in the CDW phase see below.

The excitation spectrum for the metallic phase also shows a gap. However, this does not lead to activated behaviour in the conductivity: This gap is merely the chemicalpotential gap of the charged bose liquid that constitutes the metallic phase and is a manifestation of the finite charge susceptibility at $q=0$ and $T>0$ (see Note 1).

Various correlation functions can be obtained from the Green functions that have been calculated. For example, in the nonordered phase

$$
<S^{+}(\mathbf{q}, \omega) S^{-}(\mathbf{q}, \omega)>=\frac{1}{2 \pi}(n-1) /(\omega-\epsilon(\mathbf{q})),
$$

where

$$
\epsilon(\mathbf{q})=2 B-(n-1) K_{0}-(n-1) J(\mathbf{q})=\beta^{-1} \log (n /(2-n))-(n-1) J(\mathbf{q}) .
$$

Similarly in the CDW phase

$$
<S^{+}(\mathbf{q}, \omega) S^{-}(\mathbf{q}, \omega)>=\frac{1}{\pi} \frac{\left[\omega-2 B-2 K_{0}(m+\eta)(m+\eta)\right]}{\left(\omega-2 B-K_{0} m\right)^{2}-\left[K_{0}^{2} \eta^{2}+J^{2}(\mathbf{q})\left(m^{2}-\eta^{2}\right)\right]}
$$


It is easy to check that at $\omega=0$ these correlation functions diverge as $q \rightarrow 0$ at the critical and multicritical points of the phase diagrams of Figs. III.1 and III.2. Here this divergence is characterized, of course, by mean-field exponents; corrections to these exponents and to the scaling forms for various thermodynamic and correlation functions can be obtained from renormalization-group calculations as described in Appendix D.

Given the Green functions that have been calculated, we can easily obtain the frequency-dependent conductivity by noting that the only mode of conduction in the limit of large $U$ is that in which the charged boson comprising $\mathrm{Bi}^{3+}$ moves or, equivalently, pairs of $\mathrm{Bi}^{3+}$ and $\mathrm{Bi}^{5+}$ sites are exchanged. The current operator for this mode of conduction, (Appendix E and [9]), is given by:

$$
\mathbf{j}=-i(2 e) J \sum_{<i j>}\left(S_{i}^{+} . S_{j}^{-}-H . C .\right) \mathbf{a} .
$$

By using the Kubo formula and an RPA-decoupling scheme we can evaluate the conductivity in all the phases (all effects of scattering are contained in a phenomenological scattering time $\tau$ ). The result of such a calculation for the metallic phase is of the Drude form

$$
\sigma(\omega)=\frac{n(2 e)^{2} \tau / m^{*}}{(1-i \omega \tau)},
$$

where $n$ is the number of charge-2e carriers and $m^{*}$ is the effective mass.

For the CDW phase, as $\omega \rightarrow 0$ we get $\sigma(\omega \rightarrow 0) \approx \exp \left(-\Delta / K_{B} T\right)$. (This is the anisotropy gap in the pseudospin-wave excitation spectrum in the antiferromagnetic phase.)

\section{III.3 Remarks on the viability of the Large $-U$ limit for the bis- muthates}

The phase diagram and the other results obtained above for the pseudospin model have 
many features in common with the observed properties of the barium bismuthates (Sec. III.2.1). However, there are serious limitations which, in our opinion, make this large- $U$ model not viable. The negative- $U$ Hubbard model may be a more appropriate limit for the barium bismuthates and may offer a more realistic description of these systems if we choose intermediate values of $U$.

The most serious limitation of the large- $U$ pseudospin model is that the coherence length in its superconducting phase is about one lattice spacing. This is obvious since the model corresponds to hard-core, charge-2e bosons which are onsite-paired electrons. An explicit calculation of this coherence length is given in the Appendix F. In contrast, the experimentally measured coherence length is much larger $(\simeq 80 \mathrm{~A})$.

One of the attractive aspects of the large- $U$ pseudospin model is that it yields the two gaps seen experimentally (see Secs. I and II) - an optical gap $\simeq U$ and a transport gap $\simeq 2 z V$. From the experimental data for $\mathrm{BaBiO}_{3}$ one would therefore have to infer that $U \simeq 2.0 \mathrm{eV}$ and $2 z V \simeq 0.24 \mathrm{eV}$. However, from the DOS [24] it can be estimated that (see Note 4 ) $z t=0.8 \mathrm{eV}$, so the large- $U$ assumption (i.e., $U \gg 2 z t$ ) is clearly not true. Also the charge contrast (relative to uniform $\mathrm{Bi}^{4+}$ ) implicit in the CDW phase of the large- $U$ model is too large compared to its value in these bismuthates. (The charge contrast has not been measured precisely in any experiment. However, the value of the charge contrast that can be inferred from the relative sizes of the enlarged and contracted oxygen octahedra is much smaller than \pm 1 , which the large- $U$ model predicts).

Both the shortcomings discussed above show that, if the negative- $U$ model is at all viable for these bismuthates, it is so only if the large- $U$ limit is abandoned. Thus we must look at the intermediate- and small- $U$ limits, which we discuss next in Sec.IV. 


\section{The Negative- $U$, Extended-Hubbard Model At Intermediate $U$}

\section{IV.1 Summary of Results}

In this Section we continue our discussion of the viability of modelling barium bismuthates using the negative- $U$, extended-Hubbard model (with a repulsive interaction $V$ between nearest-neighbour sites) in the intermediate- $U$ case. We obtain the phase diagram of this model via a mean-field theory and the compute the effect of fluctuations at $T=0$ in the RPA. The details of our calculations are discussed elsewhere [9, 10, 11]. We discuss only the principal results here. Furthermore, we comment on the feasibilty of using the negative- $U$, extended-Hubbard model for these bismuthates by fitting a variety of experimentally measured quantities, such as the optical and transport-activation gaps in the semiconducting phase, the superconducting $T_{c}$ and coherence length [9, 10, 11].

Our principal results for the intermediate- $U$ are:

(1) A phase diagram (Figs. IV.1a and IV.1b) for the intermediate- $U$ case, extendedHubbard model by using a mean-field approximation which is can distinguish between a variety of phases: a semiconducting, charge-density-wave (CDW) phase, superconducting phases with s-wave, extended-s-wave, or both of these types of ordering, intermediate phases with both superconducting and CDW order, and a nonordered, metallic phase. This phase diagram has the same topology as that of the large- $U$ limit (Sec.III). Furthermore, it is in qualitative accord with experimental phase diagrams in so far as it exhibits three distinct thermodynamically stable phases: a CDW phase, a singlet superconducting phase, and a nonordered metallic phase. Phase transitions between all excepting CDW and superconducting phases are continuous; the superconductor-CDW transition is first order. 
(2) If the negative- $U$ is electronic in origin (see point (6) below), then in the CDW phase there are two-particle and two-hole bound states, i.e., Cooperons, with energies in the gap of the two-particle spectrum, (Fig.IV.2b). We have calculated the pairing susceptibility within the RPA in the particle-particle channel [9, 10] to obtain these bound-state (Cooperon) energies in the CDW phase.In the limit of large $U$, this energy evolves smoothly into the anisotropy gap in the pseudospin-wave spectrum (Sec.III). The bound-state energy moves continuously towards the chemical potential as $V$ approaches zero.

(3) Charge transport in the CDW semiconducting phase is dominated by the motion of these Cooperons. The large- $U$ limit of this process reproduces the conductivity mechanism in the pseudospin model (Sec. III). Within the RPA the conductivity shows an activated behaviour with an activation energy equal to the energy of the particle-particle bound state. This is the transport gap. It is thus clearly different from the optical gap. For the latter is equal to the gap in the single-particle spectrum, i.e., it is twice the CDW gap parameter in the small- $U$ regime. At large values of $U$, this gap is $\simeq U$; note that this is not the gap in the pseudospin-wave spectrum, but is the energy required to break a pair.

(4) At large $U$ the pairs are tightly bound in real space and can be thought of as a quantum lattice gas of hard-core bosons; at small $U$ k-space pairing is the proper description of these pairs. This calculation yields a natural interpolation between these two limits at $T=0$.

(5) The conductivity in the nonordered, metallic phase is of the Drude form. At large $U$ the carriers are charge-2e, hard-core bosons (Sec. III); at small $U$ the metal is, of course, a conventional Fermi liquid.

(6) For the above explanation for the two gaps to be valid, the attractive, onsite inter- 
action must be electronic in origin: An attraction mediated by phonons is retarded and operates only over some characteristic phonon frequency. Such an interaction cannot produce the necessary binding to form the two-particle bound state as the CDW gap is $\simeq 1 \mathrm{ev}$, and any phonon-mediated attraction must be cut off well below this energy range.

The remaining part of this Section is organized as follows: In Section IV.2 we describe our mean-field and RPA calculations in the intermediate- $U$ range of the negative- $U$ Hubbard model very briefly. In Sec.IV.3 we conclude with a discussion of the strengths and weaknesses of the model, suggest future directions of study, and propose some experiments which can be used to verify our theory.

\section{IV.2 Outline of the Calculation}

\section{IV.2.1 Mean-Field Theory and The Phase Diagram}

The one-band, negative- $U$, extended-Hubbard Hamiltonian, defined on a simple cubic lattice, is given by

$$
H=-t \sum_{<i j>\sigma} c_{i \sigma}^{\dagger} c_{j \sigma}-\frac{U}{2} \sum_{i \sigma} \hat{n}_{i \sigma} \hat{n}_{i-\sigma}+\frac{V}{2} \sum_{<i j>\sigma \sigma^{\prime}} \hat{n}_{i \sigma} \hat{n}_{j \sigma^{\prime}}-\mu \sum_{i \sigma} \hat{n}_{i \sigma} .
$$

Though the qualitative features of the phase diagram of model (IV.1) are known 101, some earlier mean-field treatments [101] of it contain some errors (see Note 5). The qualitative features of the phase diagram of model (IV.1) remain the same as those of the large- $U$ model; however, there are quantitative differences.

The phase diagram (Figs. IV.1a and IV.1b) is obtained by using a mean-field approximation which is capable of distinguishing between a variety of phases: a semiconducting,

charge-density-wave (CDW) phase, superconducting phases with s-wave, extended-s-wave, 
or both of these types of ordering, intermediate phases with both superconducting and CDW order, and a nonordered, metallic phase. The order parameters used are:

$$
\begin{aligned}
& <c_{i \uparrow}^{\dagger} c_{i \downarrow}^{\dagger}>\equiv b_{s}^{*}, \\
& <c_{i \uparrow}^{\dagger} c_{j \downarrow}^{\dagger}>\equiv b_{e}^{*}, \\
& <c_{i \sigma}^{\dagger} c_{j \sigma}>\equiv C_{0},
\end{aligned}
$$

and

$$
<\hat{n}_{i \sigma}>=\frac{n}{2}+\frac{b_{c}}{2} e^{i \mathbf{Q} . \mathbf{r}_{i}}
$$

where $\mathbf{Q} \equiv \pi(1,1,1), b_{c}$ is the CDW order parameter, $b_{s}$ and $b_{e}$ are, respectively, onsite and extended-singlet-superconducting order parameters, and $\mathrm{C}_{0}$ a self-consistent contribution to the band width. Only translationally invariant order parameters are considered. A standard, Hartree-Fock decoupling, yields the mean-field Hamiltonian (we take the order parameters to be real)

$$
\begin{gathered}
H_{m f}=-\sum_{\mathbf{k} \sigma}\left(\tilde{\epsilon}_{\mathbf{k}}+\bar{\mu}\right) c_{\mathbf{k} \sigma}^{\dagger} c_{\mathbf{k} \sigma}-\frac{u_{p}}{2} \Delta_{c} \sum_{\mathbf{k} \sigma} c_{\mathbf{k}+\mathbf{Q} \sigma}^{\dagger} c_{\mathbf{k} \sigma}-\sum_{\mathbf{k}}\left(\Delta_{s}(\mathbf{k}) c_{-\mathbf{k} \downarrow} c_{\mathbf{k} \uparrow}+c_{\mathbf{k} \uparrow}^{\dagger} c_{-\mathbf{k} \downarrow}^{\dagger}\right) \\
+N\left[U b_{s}^{2}-V z b_{e}^{2}+\frac{1}{4} n^{2} u_{m}+\frac{1}{4} b_{c}^{2} u_{p}+V z C_{0}^{2}\right] .
\end{gathered}
$$

Here $N$ is the total number of lattice sites, $c_{\mathbf{k} \sigma}$ is the Fourier transform of $c_{i \sigma}, u_{m} \equiv$ $U-2 z V, \quad u_{p} \equiv U+2 z V, \bar{\mu} \equiv \mu+u_{m} n, \tilde{\epsilon}_{\mathbf{k}}=z \tilde{t} \gamma_{\mathbf{k}} ; \gamma_{\mathbf{k}} \equiv \frac{1}{z} \sum_{\mathbf{a}} \exp (i \mathbf{k} \cdot \mathbf{a})$, where $\{\mathbf{a}\}$ are the nearest neighbour lattice vectors; $\Delta_{c} \equiv u_{p} b_{c} / 2$ and $\Delta_{s}(\mathbf{k}) \equiv U b_{s}-z V b_{e} \gamma_{\mathbf{k}}$ are, respectively, the gap parameters in the CDW and superconducting phases.

This mean-field Hamiltonian (IV.3) can be easily diagonalized and the four eigenvalues are $\pm E_{\mathbf{k}}^{ \pm}$, where 


$$
\begin{gathered}
E_{\mathbf{k}}^{ \pm}=\left[R_{\mathbf{k}} \pm 2 P_{\mathbf{k}}\right]^{1 / 2} \\
R_{\mathbf{k}}=\Delta_{c}^{2}+U^{2} b_{s}^{2}+V^{2} z^{2} b_{e}^{2} \gamma_{\mathbf{k}}^{2}+\bar{\mu}^{2}+\tilde{\epsilon}_{\mathbf{k}}^{2}
\end{gathered}
$$

and

$$
P_{\mathbf{k}}=\left[\left(U b_{s} V z \gamma_{\mathbf{k}} b_{e}+\bar{\mu} \tilde{\epsilon}_{\mathbf{k}}\right)^{2}+\Delta_{c}^{2}\left(u^{2} b_{s}^{2}+\bar{\mu}^{2}\right)\right]^{1 / 2}
$$

With these four eigenvalues the mean-field free energy per site becomes

$$
\begin{aligned}
f= & -\frac{1}{N \beta} \sum_{\mathbf{k}}^{\prime}\left[\ln 4 \cosh ^{2}\left(\frac{\beta E_{\mathbf{k}}^{+}}{2}\right)+\ln 4 \cosh ^{2}\left(\frac{\beta E_{\mathbf{k}}^{-}}{2}\right)\right] \\
& +U b_{s}^{2}-V z b_{e}^{2}+\frac{1}{4} n^{2} u_{m}+\frac{\Delta_{c}^{2}}{u_{p}}+z V C_{o}^{2}+\bar{\mu}(n-1)
\end{aligned}
$$

The order parameters are determined from the self-consistency equations that follow from a minimization of this free energy. The prime over summation indicates a reduced brillouin zone. A numerical, iteration method is used to solve the self-consistency equations for the order parameters for different values of $U / z t$ and $V / z t$ ( $z$, the number of nearest-neighbour sites, is 6 in three dimensions) and at various values of filling $\delta=n-1$.

The phase diagrams in both filling-temperature and chemical potential-temperature planes are shown in Figs. IV.1a and IV.1b. The topology of the phase diagram remains the same as in the large- $U$ case, but the numbers obtained (for the positions of phase boundaries, etc.) are quite different. There are three thermodynamically stable phases: A CDW phase characterized by $\Delta_{c} \neq 0$ and $b_{s}=b_{e}=0$; separated from this CDW phase by a first-order line in the $\mu-T$ plane is the superconducting phase (characterized by $b_{s} \neq 0, b_{e} \neq 0$ and $\Delta_{c}=0$ ); and then there is a nonordered phase (all the order parameters are zero in this phase) at high temperature (at $\delta=1$ this phase goes down to $T=0)$. The phase transitions between the ordered phases and the nonordered phase are all continuous. The two lines of continuous transitions meet at a bicritical point. The firstorder line in the grand-canonical (i.e., fixed $\mu$, Fig.IV.1b) ensemble opens out into a region 
of two-phase coexistence (CDW and superconducting phases) in the canonical (i.e., fixed $\delta$, Fig.IV.1a) ensemble. $C_{0}$ is nonzero in all the phases. The hatched region in Fig.IV.1a is the two-phase coexistence region. In this two-phase region (and in equilibrium) the system phase separates into semiconducting (CDW) and superconducting phases, with one interface between them.

For $V=0$ and at half-filling (i.e., $\delta=0$ ) in model (IV.1) the CDW and superconductor solutions of the self-consistency equations are connected by a pseudospin-rotation symmetry (see Note 6) of the Hamiltonian and hence are degenerate; their free energies are equal and they coexist. Away from half filling this degeneracy is removed and the superconducting phase becomes the thermodynamically stable one: In the large- $U$ limit this corresponds to the (pseudo-spin) antiferromagnetic phase giving way to the (pseudo)spin-flop phase in a (pseudo)magnetic field. The degeneracy between CDW and superconducting phases is also lifted when $V$ becomes nonzero (the pseudospin analogue, described in Table II.2, is that the pseudospin model of Sec.III becomes anisotropic for $V \neq 0)$ : at half filling the CDW phase is the thermodynamically stable one; of course, sufficiently far away from half filling, the superconducting phase becomes stable.

The gap equation for the CDW gap parameter is of the usual BCS form, from which the CDW-nonordered transition temperature follows. The gap equation is

$$
\frac{1}{u_{p}}=\frac{1}{2} \int_{-W}^{+W} \rho(\epsilon) \frac{\tanh \frac{\beta}{2}\left[\left(\epsilon^{2}+\Delta_{c}^{2}\right)^{1 / 2}-\bar{\mu}\right]}{\left(\epsilon^{2}+\Delta_{c}^{2}\right)^{1 / 2}} d \epsilon,
$$

where $2 W$ is the noninteracting band width. At half filling $\bar{\mu}=0$ and, if we take the density of states $\rho(\epsilon)$ to be a constant, this becomes the usual BCS gap equation. At $T \equiv 1 / \beta=0$ and with a constant DOS this gives the CDW gap parameter $\Delta_{c}=W / \sinh \left(2 W / u_{p}\right)$

For the superconducting phase the calculations are also of the BCS type. The gap equation now involves the $2 \times 2$ matrix equation: 


$$
\tilde{M}=\left[\mathbf{1}-\frac{1}{2 N} \sum_{k}^{\prime} \frac{\tanh \left(\tilde{\epsilon}_{\mathbf{k}}+\bar{\mu}\right)}{\left(\tilde{\epsilon}_{\mathbf{k}}+\bar{\mu}\right)}\left(\begin{array}{cc}
U & -V z \gamma_{\mathbf{k}} \\
U \gamma_{\mathbf{k}} & -V z \gamma_{\mathbf{k}}^{2}
\end{array}\right)\right] .
$$

where 1 is the $2 \times 2$ unit matrix. The determinant of the matrix $\tilde{M}$ should vanish at $T_{c}$. Note that, in this mean-field theory, the maximum superconducting $T_{c}$ occurs at the bicritical point.

\section{IV.2.2 The Cooperon Bound State In The Random Phase Approximation}

We showed in Sec.III that the experimental observation [2] of well-separated optical and transport gaps can be explained by identifying the optical gap with the pair-breaking energy and the transport gap with the pseudospin-wave gap in the CDW semiconducting phase. This explanation remains viable even in case of the negative-U, extendedHubbardmodel for intermediate $V$.

As in the large- $U$ case the CDW phase continues to show two well-separated gaps for intermediate values of $U$ and $V$. The physics of these two gaps is as follows: Our mean-field theory yields quasiparticle valence and conduction bands with a gap separating them, (Fig.IV.2a). In k-space this gap opens up along the entire noninteracting Fermi surface. In the ground state, the valence band is full whereas the conduction band is empty ( at half filling, i.e., for pure $\mathrm{BaBiO}_{3}$ ) and this system is insulating. Now consider putting two electrons into the conduction band. They still feel the residual attraction $U$ (modified by appropriate coherence factors in general). Hence they form a bound state (a Cooperon, i.e., a charge-2e boson) in the gap in the two-particle excitation spectrum, (Fig.IV.2b). Two holes in the valence band form a similar bound state. This bound state forms provided the source of the attractive $U$ is electronic [18] and not phononic since $U$ must remain attractive over energy scales larger than the CDW gap $\Delta_{c}$, which is 1 $\mathrm{eV}$, as measured from the Fermi level. Also a phononic mechanism cannot give rise to a binding of nearly $0.8 \mathrm{eV}$ (required to fit experimental observations; see below). It is easy 
to see that charge (and all other) transport in this CDW (semiconducting) phase will be dominated by these Cooperons. The thermal activation energy required for exciting these Cooperons is the gap for exciting two quasiparticles minus the binding energy of these Cooperons (i.e., the energy of the bound state measured from the chemical potential) (Fig.IV.2b). Hence it is lower than (half) the semiconducting (CDW) gap 2 $\Delta_{c}$. On the other hand, optical experiments, which excite single particles across the CDW gap, measure this larger gap, $\Delta_{c}$.

The existence of a two-particle bound state in the CDW phase is indicated by the appearence of a pole in the pair susceptibility in the Cooper channel (at wave vector $\mathbf{q}=0$ ) below the bottom of the continuum of the two-particle excitation spectrum (the two-particle continuum starts at $2 \Delta_{c}$ (Fig.IV.2b).

The (singlet) pair susceptibility in the Cooper channel is defined by

$$
\begin{gathered}
\chi_{p, i j ; i^{\prime} j^{\prime}}(\mathbf{q}, \nu)=\frac{1}{2 \pi N^{2}} \\
\times \int_{-\infty}^{+\infty} d \tau \exp (i \nu \tau) \sum_{\mathbf{k}, \mathbf{k}^{\prime}}^{\prime}\left\langle T_{\tau}\left[c_{\mathbf{k}+\mathbf{q} \uparrow, i^{\prime}}(\tau) c_{-\mathbf{k} \downarrow, j^{\prime}}(\tau) c_{\mathbf{k}^{\prime}+q \uparrow, i}^{\dagger}(0) c_{-\mathbf{k}^{\prime} \downarrow, j}^{\dagger}(0)\right]\right\rangle
\end{gathered}
$$

where $T_{\tau}$ represents time-ordering, $i, j$, etc. are band indices (in the CDW ground state there are two bands), the subscript $p$ stands for pairing, and the expectation value is calculated with respect to the interacting ground state. We restrict ourselves to $\mathrm{T}=0$.

We show in Fig.IV.3 the RPA ladder diagrams for the pair susceptibility $\chi_{p, i j ; i^{\prime} j^{\prime}}(\mathbf{q}, \nu)$. We have calculated the sum of these diagrams [9, 10, 11] in the presence of a CDW background and starting from a negative- $U$, extended-Hubbard model at half filling. From this the two-particle (hole) excitation spectrum is obtained, and thence the location of the Cooperon bound state. It is shown that, as long as $V$ (more precisely, $\Delta_{c}$ ), is small which is satisfied for the barium bismuthates because the $\Delta_{c} \simeq 1 \mathrm{eV}$ ), the bound state falls within the gap of the two-particle spectrum (Fig.IV.2b) and, for any nonzero $V$, this state lies above the chemical potential (all energies are measured from the chemical 
potential). Hence, so long as $V \neq 0$, the CDW phase is not unstable with respect to the superconducting phase at half filling.

We show that the resulting two-particle bound-state energy in the intermediate- $U$ regime evolves into the pseudospin-wave gap of the large- $\mathrm{U}$ model. It is interesting to note that the RPA excitation spectrum calculated at intermediate values of $U$ evolves into the RPA pseudospin-wave excitation calculated in the large- $U$ limit (for it is not evident a priori that the two RPA's are equivalent in all respects).

In the presence of a CDW background we have done the analogue of the Cooperinstability [102 calculation by following the t-matrix approach [103]. This requires the solution of a secular equation involving a $14 \times 14$ matrix [9, 10]. Had it been possible to cast this equation into an equation of the type $1 / \lambda=\Phi(\nu)$ (where $\lambda$ is some effective, attractive interaction), then its solutions could have been portrayed as shown schematically in Fig.IV.4, i.e., as the intersections (empty circles) of the horizontal line $(y=1 / \lambda$, where $\lambda$ is negative since the residual interaction is attractive) with the curves (thick lines) representing $\Phi(\nu)$. The vertical lines (thin lines) are the unperturbed CDW eigen-energies starting from $\pm 2 \Delta_{c}$. We have taken the system to be finite system for the purposes of this figure, so the energy levels are discrete.As the system size goes to infinity, the eigenenergies of the interacting system which fall above $2 \Delta_{c}$ or below $-2 \Delta_{c}$ form the two-particle and two-hole continua respectively, and correspond to scattering states; however, the two eigenenergies inside the gap remain separated from these continua, and correspond to the bound states (the one below the chemical potential is the two-hole bound state and the one above is the two-particle bound state).

The energy of the bound state depends on $U, V$ and $z t$. In order to pin down at least some of the parameter values, we proceed as follows:

(1) Set $\Delta_{c}=1 \mathrm{eV}$, since the optical gap, which is twice the CDW gap parameter, is $2 \mathrm{eV}$ for $\mathrm{BaBiO}_{3}$. (We use the mean-field gap equation and a constant density of states which 
makes $\Delta_{c}=z \tilde{t} / \sinh \left(2 z \tilde{t} / u_{p}\right)$, where $u_{p}=U+2 z V$ and $z \tilde{t}$ is half the band width.)

(2) The Cooperon bound-state energy (measured from the chemical potential) is set equal to the transport gap of $\mathrm{BaBiO}_{3}$, i.e., $0.24 \mathrm{eV}$. The values of $U$ (both screened and unscreened; see below) and $V$ required to satisfy these two conditions for different choices of the values of $z t$ are plotted in Figs. IV.5a and IV.5b. The main results obtained from the above calculations, relevant to the modelling of the barium bismuthates, are summarized below:

(1) For the potassium-doped system the band width, close to the metal-semiconductor transition is roughly $1.6 \mathrm{eV}$, as obtained from the DOS (Batlogg, et al 24]). Given this value we obtain, from Figs.IV.5a,b $U=1.9 \mathrm{eV}$ and $2 z V=0.13 \mathrm{eV}$. Clearly then we must consider the intermediate- $U$ and not the large- $U$ case.

(2) The two-particle bound-state energy goes to zero continuously as $V$ approaches zero. At $V=0$ there is a gapless two-particle mode that signals the instability of the CDW state towards superconducting order. In Fig.IV.6 we show the values of the interaction-parameters obtained from the two conditions described above, for a fixed value of $W(=z t)$, as a function of the bound-state energy. Note that the extendedCoulomb term $V$ goes to zero as the energy of the bound state approaches the chemical potential.

(3) The bound-state energy goes to $2 z V$ as the band width approaches zero (Fig.IV.5b). Note that in the large- $U$ limit the anisotropy gap is exactly equal to $2 z V$, a feature that is reproduced correctly here as $z t \rightarrow 0$. This shows that the above results interpolate smoothly between the small- and large- $U$ results for the extended-Hubbard model at $T=0$.

We now compare our results with experimentals. However, we cannot naively use our estimates for the values of $U$ and $V$ to calculate the superconducting and CDW 
transition temperatures for the following reasons: Our estimates of $U$ and $\mathrm{V}$ have been made at the extreme insulating limit of $\mathrm{BaBiO}_{3}$, whereas superconductivity and the metalsemiconductor transition occur after significant doping ( $\delta \simeq 0.4$ for the potassium-doped system). We must, therefore, try to incorporate the effects of metallic screening on the strength of the Coulomb interaction. In addition, doping has another important effect on these systems. The arguments used for an attractive, onsite interaction were based on nonlinear screening of the ionised configurations of the Bi atoms by the highly polarizable O octahedra. It is conceivable that, with increased doping, as the system moves towards the metallic side and the lattice distortions and the charge-disproportionated, highly ionized Bi configurations begin to disappear, the mechanism which leads to the attractive interaction gets suppressed rapidly. At this stage it is difficult to calculate these effects, as we do not have a first-principles theory for the negative value of $U$.

In order to account for at least some of the physical processes that result from doping, we have included the effect of metallic screening at the simplest level via the RPA. The corresponding RPA screening diagrams are shown in Figs.IV.7a and IV.7b (repeated excitations of particle-hole or particle-particle pairs, out of the Fermi sea ). Note that screening operates on both the particle-particle and particle-hole channels. Hence the effective interactions responsible for CDW and superconductivity are both reduced. Using the standard RPA procedure to calculate the t-matrix we write the corresponding effective (screened) interaction as $\mathcal{V}_{s c}(\mathbf{q})=\mathcal{V}(\mathbf{q}) /\left[1-\mathcal{V}(\mathbf{q}) \chi_{0}(\mathbf{q})\right]$ where $\mathcal{V}$ is the bare interaction and $\chi_{0}(\mathbf{q})$ is the appropriate bare susceptibility (particle-particle or particle-hole). Now the particle-hole susceptibility at wave vector $\mathbf{Q}$ is the same as particle-particle susceptibility at zero wave vector. Hence, in the simplest approximation, we can replace $\chi_{0}$ by the density of states $N(0)$ at the Fermi level and get the effective screened interaction governing the CDW or the superconducting transition. For $N(0)$ we use the experimental value at the metal-semiconductor boundary of the potassium-doped system. 
By using the above procedure to account for screening, we plot the results for the superconducting and CDW transition temperatures in Figs.IV.8a and IV.8b calculted via the BCS formula discussed earlier (with both bare and screened interactions), as a function of the band width. The results for the phase boundaries obtained using this procedure are discussed in Appendix $\mathrm{C}$ and shown in Fig.IV.9. Note that we have used screening corrections only for the on-site Coulomb term. A calculation of the t-matrix for the momentum dependent $V$ term is rather complicated and is not done here. Since the values of $V$ we are interested in are quite low (Fig.IV.9) compared to $U$, it is reasonable to neglect the screening corrections to $V$. Indeed the dashed line in Fig.IV.9 shows the superconductor-nonordered phase boundary with $V=0$ (we show the results for two different set of parameters values, and note that the parameter values that are appropriate for the bismuthates correspond to Fig.IV.9). This shows the negligible effect $V$ has on $T_{c}$ in the range of interactions that we are interested in. In passing we note that the effect of $V$ on the superconducting $T_{c}$ vanishes as $\delta$ approaches zero [9, 10].

In any case, our results clearly show that the values obtained for the superconducting and CDW transition temperatures are too high compared to the experimental values. The effects of fluctuations neglected in our mean-field theory and the effects of disorder could reduce these transition temperatures by as much as a factor of 4 , but probably not much more. It is likely that the reduction of the strength of attraction with doping (as described earlier) must be incorporated in our theory order to get better experimental fits for the $T_{c}$. (This especially for the superconducting transition temperature; the mean-field, CDW transition temperature is not orders of magnitude off from the experimental value, lending support to our hypothesis that, as doping increases, the negative- $U$ is reduced.)

In spite of this limitation of large transition temperatures, our theory yields quite a few interesting results. Firstly, the large difference between the transport gap the optical and gap can be understood from the existence of the two-particle bound state in the 
CDW phase. The conductivity is given by $\sigma(\omega)=\left(n e^{* 2} \tau / m^{*}\right) /(1-i \omega \tau)$, where $m^{*}$ is the effective mass of the charge carriers, i.e., the Cooperons and $e^{*}=2 e$ is their charge. $n(T) \approx \exp \left(-E_{A} / K_{B} T\right)$ is the number of charge-2e carriers at temperature $T$, where $E_{A}$ is the transport gap. Hence $\sigma(\omega \rightarrow 0)$ shows an activated behaviour with an activation energy $E_{A}$. Secondly, this theory clearly reproduces some of the qualitative features of the phase diagram for the bismuthates (see the concluding discussion below). In addition, the value of $2 \Delta / K_{B} T_{c}$ is 3.5 , and the system is diamagnetic at low temperatures in our model. We have taken the standard mean-field expression for the superconducting coherence length and used the observed values of the Fermi velocity to obtain a coherence length of nearly $30 \AA$. Since our calculation grossly overestimates the superconducting $T_{c}$, it is not surprising that our estimate for the coherence length is quite small compared to the experimental value. Nevertheless, it is much better than the extremely short coherence length predicted by the large- $U$ (Sec.III) where the superconductivity arose from onsite paired electrons. Here, in the intermediate- $U$ case, the pairing is in momentum space and the pairs are spatially extended. 


\section{IV.3 Concluding Discussions}

In this and the preceding Sections we have investigated the negative- $U$, extendedHubbard model to model the barium bismuthate superconductors. In our study we have assumed that the mechanism that leads to the negative value of $U$ is electronic rather than phonon mediated. At the end of studying both large- and intermediate- $U$ ranges we have been successful in understanding some of these features associated with the bismuthate systems.

Perhaps the most important aspect of our treatment is the detailed theory we have provided for the occurence of two gaps in the semiconducting phase of the barium bismuthates. We have identified the transport gap as the energy of the two-particle bound state (Cooperon) and the optical gap as twice the CDW gap. This picture of the two gaps is consistent with the fact that the transport gap is not easily accessible to optical reflectance, photo-acoustic or photoconductivity studies, all of which involve single particle excitations. Note that the above picture implies that the semiconducting phase of the bismuthates is unique, with transport properties dominated by charge-2e bosons.

Indeed, by fitting the theoretical gaps to the experimental ones (Sec.IV.2) we have estimated the interaction parameters of the system. With these parameter values we have calculated other normal-state and superconducting properties. We have set up the formalism for the calculation of the particle-particle excitation spectrum in the RPA for the extended-Hubbard model in the CDW phase. In addition, we have demonstrated that a smooth interpolation between the large- and small- $U$ theories is possible at $T=0$, and that the particle-particle excitation spectrum we calculate at intermediate $U$, evolves smoothly into the correct pseudospin-wave spectrum in the large- $U$ limit. For typical intermediate values of $U$, and $V$ (i.e., when $U \leq 2 z t$ and $V<U$ ) our phase diagram in the $T-x$ plane (Fig.IV.1a), has the same qualitative features as the large- $U$ phase diagram (Fig.III.1) and shares many features with experimental phase diagrams. Furthermore, the 
charge-contrast in the CDW phase gets reduced and the superconducting coherence length enhanced over the values obtained in the large- $U$ limit, in slightly better accord with the experiments. We have also argued that the processes that give rise to the attractive interaction, if electronic in origin, must become less and less effective as the system is doped towards the metallic side, so the strength of the attractive interaction ought to be reduced in order to obtain the experimentally observed superconducting $T_{c}$.

It is worth stressing that our theory disfavours a purely phonon-mediated mechanism for the negative $U$ (unless an alternative explanation can be found for the well-separated optical and transport gaps). A phonon mediated-attraction is necessarily retarded (cut off at characteristic phonon frequencies $\simeq 100 \mathrm{~K}$ ); the minimum energy range over which the attraction must operate, in order to produce the two gaps, is about $1 \mathrm{eV}$. In addition, the large binding required to produce the two-particle bound state at about $0.24 \mathrm{eV}$ above the chemical potential cannot come from phononic mechanism. It has been observed recently (Hinks, et al., [14, 15]) that calculations based on realistic phonon these systems can, at least at the level of molecular-dynamics simulations, produce rather large superconducting $T_{c}$, without requiring a very strong electron-phonon coupling $\left(\lambda_{e-p}\right.$ around 0.6-0.8) or a large DOS at the Fermi level. The correct scenario could be that in the semiconducting phase of these systems, the attraction is mainly electronic in origin; however, this electronic component of the attraction becomes weak as the system is driven towards the metallic side (see discussion above), and the electron-phonon induced attraaction also becomes important in the superconducting phase.

According to our theory the carriers responsible for charge transport in the semiconducting phase of the bismuthate systems are charge-2e bosons (Cooperons). Hence experiments that can pick up the charges of carriers in the semiconducting phase would be of great value in verifying our theory. Unfortunately the determination of the charge of carriers in pure transport measurements is very difficult; they generally yield only the 
ratio of the effective charge and effective mass.

However, the following is worth looking into. Stanton and Wilkins [104 have observed that the noise spectrum of the high-field transport in a semiconductor is $S(\omega, E)=$ $\left(n e^{* 2} \tau_{0}\right) /\left(1+\left(\omega \tau_{0}\right)^{2}\right) \times(A / 2 L)\left[k_{B} T_{0} / m^{*}+\left(e^{*} E \tau_{0} / m^{*}\right)^{2}\right]$, where $E$ is the external electric field, $T_{0}$ is the equilibrium lattice temperature, $\tau_{0}$ is the phenomenological relaxation time of the charge carrier of effective charge $e^{*}$ and mass $m^{*}$, and $A$ and $L$ are respectively the area and length (in the direction of transport) of the sample. Now if the thermaland electric-field dependent components of the noise spectrum could be separated, the above expression for $S(\omega, E)$ would allow us to calculate the charge of the carriers and their effective mass separately. To measure the noise spectrum, however, one must do a very controlled experiment at low temperatures on a pure, single-crystal sample of $\mathrm{BaBiO}_{3}$. A measurement of the phase change on passing through a magnetic field (BohmAharanov effect) can also, in principle, detect the charge of the carrier, but such an experiment on mesoscopic samples of $\mathrm{BaBiO}_{3}$ may be difficult to perform and hard to interpret. Even more likely possiblilties for observing the charge-2e Cooperon may be in tunnelling measurements from a superconductor into the $\mathrm{BaBiO}_{3}$ semiconductor, since pair tunnelling should now become viable.

We believe that such experimental work that confirms or rules out this picture of the semiconducting phase of the barium bismuthates would be of great interest. A confirmation of this transport mechanism in the semiconducting phase of the barium bismuthates would confirm the electronic origin, as opposed to the phononic origin, for the negative $U$.

We note that the phase diagram (Fig.IV.1), obtained for the negative- $U$, extended Hubbard model in our mean-field theory does not contain any intermediate phase (i.e., one with both CDW and superconducting order), unlike the mean-field phase diagram of Micnas, et al 80]. In the large- $U$ case earlier work (Liu and Fisher 95] and Matsuda and 
Tsuneto 96]) has shown clearly that, in mean-field theory, such a phase is possible only in the presence of longer range of interactions.

Needless to say, in spite of the attractive features discussed above, the negative- $U$ extended-Hubbard model, as applied to these bismuthates, has its limitations. The main shortcomings arise because we have neglected electron-phonon interactions,the longrange part of the Coulomb interactions, and disorder (charge disorder in the case of $\left.\mathrm{Ba}_{1-x} K_{x} \mathrm{BiO}_{3}\right)$. We comment on these shortcomings below.

The neglect of Coulomb interactions is probably the main reason for one important discrepancy between the experimental phase diagram (Fig.I.2b) and the one obtained (Fig.IV.1) from the model discussed above: The former has no two-phase region for $x<0.4$, unlike the latter. Since the CDW and the superconducting phases have different average Bi-occupancies (or charges), long-range, Coulomb interactions strongly disfavour any bulk phase separation. Instead they might favour the formation, for small deviations from half-filling, of a new phase consisting of a dispersion of globules of superconducting material (which in the large- $U$ limit and with small enough globule sizes can be thought of as discommensurations, citeCMV) inside a semiconducting CDW background. If the background compensating charge (because of potassium ions) were smeared uniformly, one might expect this dispersion to be a periodic array. However, in the actual system the potassium ions are distributed randomly, so the dispersion might well be random. Such a phase would be semiconducting above a new critical temperature (much lower than the critical temperature for the bulk superconductivity) corresponding to the destruction of the phase coherence between the different superconducting globules ( this temperature would be determined by the weak Josephson coupling between the globules). It is interesting to speculate that the semiconducting region, observed experimentally (Fig.III.2b) away from half-filling, corresponds to such a dispersed phase.

Another possibility that one ought to explore, both experimentally, and theoretically, 
even in the nearest-neighbour model (IV.2) and the mean-field framework, is whether the first-order coexistence region Fig.IV.1b gets replaced by an incommensurate phase, or a succession of commensurate phases.

We must also account for the effects of disorder: In the potassium-doped case since $\mathrm{K}^{1+}$ replaces $\mathrm{Ba}^{2+}$, in addition to one electron being removed from the system (which we have considered in our model), a random Coulomb potential is also introduced ( $\mathrm{K}$ is effectively a $1^{-}$impurity). In the simple theory presented here, we have neglected this random potential. The effect of randomness is even more serious in lead-doped $\mathrm{BaBiO}_{3}$ since the $\mathrm{Pb}$ atoms remove the negative- $U$ centers of $\mathrm{Bi}$ randomly. Only very simple studies of the effects of this randomness on CDW ordering have been carried out so far (Jurczek and Rice [79] and Jurczek 83]).

Since $\mathrm{BaPbO}_{3}$ is a metal, it is clear that for a proper study of the entire range of lead doping, the one-band model (II.2) is inadequate and the more complex model (II.1) has to be used. Also the electron-phonon interactions are strong in these systems and has to be treated in a better way than has been done so far, especially because of the structural transitions in these systems. Clearly more detailed experimental and theoretical studies are called for to address these issues.

It is worth noting in the end that there are some other theoretical attempts recently using negative- $U$, extended Hubbard model to understand the bismuthates. Ohkawa 105 has studied a negative- $U$, extended-Hubbard model using $1 / d$ expansion in the slave boson approach [106]. The leading order effect in $1 / d$ produces a "kondo" like "charge fluctuation" regime discussed earlier in the context of an attractive impurity model [84]. A mean-field analysis of this model produces, at a lower temperature, both CDW and superconducting phases as we have shown above [9, 10]. Ohkawa argues that the normal state of barium potassium bismuthate represents a diamagnetic heavy electronic system with a large $\left(\simeq 3-4 \times 10^{3} \mathrm{~K}\right)$ charge-kondo temperature $T_{K}$. Superconductivity occurs 
in a band of width $4 T_{K}$ in the usual BCS route (via Cooper pairing), with the enhanced mass of the electrons leading to high $T_{c}$. A three band, negative- $U$ extended Hubbard model has also been considered by Bala and Oles [107] recently leading to a phase diagram similar to ours (as described in Sec.IV).

\section{Acknowledgements}

We thank the University Grants Commission and the Council of Scientific and Industrial Research in India for research support. The Supercomputer Education and Research Centre at Indian Institute of Science, Bangalore is acknowledged for computational support. Two of us (TVR and AT) would like to thank IFCPAR-408-2 for partial support for work done in France. AT acknowledges helpful discussions with B. K. Chakraborty, F. Cyrot-Lackmann, C. Escribe-Filippini, D. M. Gaitonde and S. Sarker. AT also acknowl-

edges support from Jawaharlal Nehru Centre for Advanced Scientific Research, Bangalore. 


\section{Appendices}

\section{Appendix A}

\section{The Pseudospin Hamiltonian as the large- $U$ limit of the Hubbard Model}

There are various ways of transforming the Hubbard model to a spin model in the large- $U$ limit. We use most straightforward route via second-order, degenerate, perturbation theory, which is also physically transparent. Other methods use a canonical transformation [108, 109].

Consider the negative- $U$ Hubbard model

$$
H=-t \sum_{<i j>\sigma} c_{i \sigma}^{+} c_{j \sigma}-U \sum_{i}\left(n_{i}-1\right)^{2}
$$

In the large- $U$ limit, the first term can be taken as a perturbation and one can construct an effective Hamiltonian describing the low-energy physics.

The full Hilbert space consists of four states per site: the zero-occupancy, doubleoccupancy and single-occupancy (with up and down spins) states denoted by $|0>| 2>$,,

$\mid \uparrow>$ and $\mid \downarrow>$, have energies $-U,-U$ and 0 respectively. The degenerate, lower energy states with double and zero occupancy are separated from the higher energy doublet of singly occupied states by the energy $U$. A chemical potential term lifts the degeneracy between the low energy doublet $\mid 0>$ and $\mid 2>$. However, as long as $U \gg \mu$, one can do a degenerate perturbation calculation involving the nearly degenerate lower energy manifold of unoccupied and doubly occupied states (see below).

We now use degenerate perturbation theory to obtain the lowest-order (second order in $t$, as the first-order term vanishes) effective Hamiltonian in the subspace of states $|0\rangle$ and $\mid 2>$, with the singly occupied states $\mid \uparrow>$ and $\mid \downarrow>$ being virtual (intermediate) states. 
It is clear that at this order, only the nearest-neighbour sites will be connected by the perturbation and so we choose a direct-product basis in the site representation: $\mid i 0 ; j 0>$ , $|i 0 ; j 2>| i ,2 ; j 0>, \mid i 2 ; j 2>$ where $\mid i 0 ; j 0>$ is the state with zero occupancy at both $i$ and $j$ sites, etc. The projected Hamiltonian in this subspace is

$$
H=4 \frac{|t|^{2}}{U}(|i 0 ; j 0><i 0 ; j 0|+|i 2 ; j 2><i 2 ; j 2|)+4 \frac{t^{2}}{U}(|i 0 ; j 2><i 2 ; j 0|+\text { h.c. }) .
$$

Now we define the operators

$$
\begin{gathered}
S_{i}^{+} \equiv|i 2><i 0|, \\
S_{i}^{-} \equiv|i 0><i 2|, \\
S_{i}^{z} \equiv \frac{(|i 2><i 2|-|i 0><i 0|)}{2}
\end{gathered}
$$

where $\mid i 2>$ represents the state with 2 electrons at the $i$-th. site and $S_{i}^{-}=\left(S_{i}^{+}\right)^{\dagger}$.

These operators obey the commutation relations of spin operators and hence are called pseudospin operators. Note that $S_{i}^{z}=\frac{1}{2}$ implies that the i-th site is a doubly occupied site while $S_{i}^{z}=-\frac{1}{2}$ implies it is unoccupied.

In terms of these operators the effective Hamiltonian becomes

$$
H_{p s e u d o s p i n}=J \sum_{<i j>}\left(\mathbf{S}_{i} \cdot \mathbf{S}_{j}-\frac{1}{4}\right)
$$

where $J=4 t^{2} / U$.

This is an isotropic Heisenberg Hamiltonian. Deviations away from half filling, modelled by a chemical potential $\mu \neq U$, will break the pseudospin-rotation invariance [84] and lift the degeneracy between the states $\mid 0>$ and $\mid 2>$. So long as $U \gg \mu$, the corrections to the results of degenerate perturbation theory because of $\mu$ are $\frac{t^{2}}{U} \cdot \frac{\mu}{U}$ i.e. they are clearly higher order in $\frac{1}{U}$. The term $V \sum_{<i j>} n_{i} n_{j}$, when projected onto the subspace of 
$\mid 0>$ and $\mid 2>$, takes the form $4 V \sum_{<i j>}\left(S_{i}^{z}+\frac{1}{2}\right)\left(S_{j}^{z}+\frac{1}{2}\right)$, which also breaks the pseudospin rotation symmetry.

Thus the final effective Hamiltonian in the projected subspace is

$$
\mathcal{H}_{\text {pseudospin }}=J \sum_{<i j>}\left(\mathbf{S}_{i} . \mathbf{S}_{j}-\frac{1}{4}\right)+4 V \sum_{<i j>} S_{i}^{z} S_{j}^{z}-B \sum_{i}\left(2 S_{i}^{z}+1\right),
$$

where $B=\mu+U-2 z V$ and $z$ is the coordination number of the lattice.

\section{Appendix B}

The Relation Between Pseudomagnetic Field and Magnetization in the Different Mean-Field Phases and the Phase Boundaries Across Them

To obtain the pseudomagnetic field (and hence the chemical potential) in the different mean-field phases obtained by the mean-field theory of the pseudospin model (Sec. III.2), we use the following thermodynamic identity

$$
-\partial f / \partial B=n=<S_{i}^{z}>+<S_{j}^{z}>+1
$$

The subscripts $i$ and $j$ label sites on the two different sublattices A and B (or B and A). This equation, in conjunction with the self-consistency equations (Sec.III.2) yield the pseudomagnetic field in in terms of the magnetisation $m$.

\section{B.1 Pseudomagnetic Field}

\section{Superconducting Phase}

In the superconducting phase $<S_{A}^{z}>=<S_{B}^{z}>\equiv<S^{z}>$ and $\left|<S_{A}^{x}>\right|=\left|<S_{B}^{x}>\right|$. Hence $R_{A}=R_{B} \equiv E$ (See Section III). Thus equation (III.2) gives 


$$
B=m /\left(\frac{\tanh ^{-1}(\beta E)}{E}\right)+K_{0}<S^{z}>
$$

where $m=n-1$, is the magnetization, $J_{0}=z J$ and $K_{0}=z K$.

From the self-consistency equation (Sec.III)

$$
<S_{A(B)}^{x}>=\frac{J_{0}}{2 R_{B(A)}}<S_{B(A)}^{x}>\tanh \left(\beta R_{B(A)}\right) .
$$

For the superconducting phase

$$
J_{0}^{-1}=\frac{\tanh (\beta E)}{2 E}
$$

By combining equations (B2) and (B3), we get

$$
B=\left(K_{0}+J_{0}\right) m / 2
$$

in the superconducting phases.

\section{Nonordered Phase}

Similarly for the nonordered phase $\left(<S_{A(B)}^{x}>=0\right.$ and $\left.<S_{A}^{z}>=<S_{B}^{z}>\equiv<S^{z}>\right)$ whence

$$
B=1+\beta^{-1} \tanh ^{-1} m+\frac{k_{0}}{2} m
$$

\section{Charge-Density-Wave Phase}

Here $<S_{A}^{x}>=<S_{B}^{x}>=0$. So, following the procedure outlined above we get

$$
m=1 / 2\left[\tanh \beta\left(B-K_{0} / 2(m-\eta)\right)+\tanh \beta\left(B-K_{0} / 2(m+\eta)\right)\right]
$$

where $\eta=<S_{A}^{z}>-<S_{B}^{z}>$ 


\section{B.2 Phase Boundaries}

\section{Charge-Density-Wave - Nonordered}

The CDW-nonordered phase boundary is obtained by noting that the staggered magnetisation $\eta=<S_{A}^{z}>-<S_{B}^{z}>$ goes to zero continuously as this boundary is approached from the CDW side. By expanding the self-consistency equations about this boundary $(\eta \rightarrow 0)$ we get

$$
k_{B} T_{C N}=\frac{K_{0}}{2}\left(1-m^{2}\right) .
$$

where $T_{C N}$ is the transition temperature.

\section{Superconductor-Nonordered}

Here the order parameter $\left\langle S^{x}>\rightarrow 0\right.$ as the phase boundary is approached from the low-temperature side. An expansion of the self-consistency equations yields

$$
B=K_{0} m / 2+J_{0} \tanh \beta\left(B-K_{0} m\right)
$$

which along with Eq.(B4) gives

$$
T_{S N}=J_{0} m /\left(k_{B} \log [(1+m) /(1-m)]\right)
$$

\section{Appendix C}

\section{The Equation of Motion for the Green Function in the RPA}

To obtain the excitation spectra in different mean-field phases, we used a RandomPhase-Approximation technique [9, 80] for the closure of the equations of motion for the 
Green function (Sec. III). We use the Hubbard operators $100 \hat{P}_{\alpha \beta}^{i} \equiv|i \alpha><i \beta|$. In the models we discuss $\alpha$ and $\beta$ can take two values ( + and - ) corresponding to the two mean-field eigenstates $\left|+>=\cos \frac{\theta}{2}\right| \uparrow>+\sin \frac{\theta}{2} \mid \downarrow>$ and $\left|->=\cos \frac{\theta}{2}\right| \downarrow>-\sin \frac{\theta}{2} \mid \uparrow>$. The indices $i$ label sites on a bipartite lattice; with nearest-neighbour interactions, the only relevant indices are the two sublattice labels (A and B). The commutation relation between these Hubbard operators is

$$
\left[\hat{P}_{\alpha \alpha^{\prime}}^{i}, \hat{P}_{\beta \beta^{\prime}}^{i}\right]_{-}=\delta_{i j}\left(\hat{P}_{\alpha \beta^{\prime}}^{i} \delta_{\alpha^{\prime} \beta}-\hat{P}_{\alpha^{\prime} \beta}^{i} \delta_{\alpha \beta^{\prime}}\right)
$$

where $[\hat{R}, \hat{S}]_{-}$represents a commutator between $\hat{R}$ and $\hat{S}$. In terms of these projection operators the double-time, retarded Green function $G_{\alpha \beta, \gamma \delta}^{m, n}\left(t-t^{\prime}\right.$ ) (also denoted by $<\hat{P}_{\alpha \beta}^{m}(t) \mid \hat{P}_{\gamma \delta}^{n}\left(t^{\prime}\right)>>$, [110] is

$$
G_{\alpha \beta, \gamma \delta}^{m, n}\left(t-t^{\prime}\right)=-i \theta\left(t-t^{\prime}\right)<\left[\hat{P}_{\alpha \beta}^{m}(t), \hat{P}_{\gamma \delta}^{n}\left(t^{\prime}\right)\right]_{-}>
$$

This Green function gives the probability amplitude for the process in which, at time $t^{\prime}$, the $n^{\text {th }}$ site makes a transition from the state $\mid n \delta>$ to the state $\mid n \gamma>$ followed by another transition, at site $m$ and a later time $t$, from the state $\mid m \beta>$ to the state $\mid m \alpha>$. The Fourier transform of this Green function in time is

$$
G_{\alpha \beta, \gamma \delta}^{m, n}(\omega)=\frac{1}{2 \pi} \int_{-\infty}^{\infty} d t G_{\alpha \beta, \gamma \delta}^{m, n}\left(t-t^{\prime}\right) \exp i \omega\left(t-t^{\prime}\right)
$$

Correlation functions of two projection operators are expressed in terms of the Green function in the usual way:

$$
<\hat{P}_{\gamma \delta}^{m}\left(t^{\prime}\right) \hat{P}_{\alpha \beta}^{n}(t)>=\int_{-\infty}^{\infty} d \omega\left[G_{\alpha \beta, \gamma \beta}^{m, n}(\omega-i 0)-G_{\alpha \beta, \gamma \beta}^{m, n}(\omega+i 0)\right] f_{B}(\omega)
$$

where $f_{B}(\omega)$ is the Bose occupation factor $\left(\exp \omega / k_{B} T-1\right)^{-1}$. 
Since any local operator can be represented in terms of the projection operators as $A_{i}=\sum_{\alpha \beta}\left(A_{i}\right)_{\alpha \beta} \hat{P}_{\alpha \beta}^{i}$, the pseudospin Hamiltonian can be written as

$$
H=-\sum_{i, \alpha \beta} u_{\alpha \beta}^{i} \hat{P}_{\alpha \beta}^{i}-\sum_{i j, \alpha \alpha^{\prime}, \beta \beta^{\prime}} W_{\alpha \alpha^{\prime}, \beta \beta^{\prime}}^{i j} \hat{P}_{\alpha \alpha^{\prime}}^{i} \hat{P}_{\beta \beta^{\prime}}^{j}
$$

where

$$
u_{\alpha \beta}^{i}=B\left[2\left(S_{i}^{z}\right)_{\alpha \beta}+1\right]
$$

and

$$
W_{\alpha \beta, \gamma \delta}^{i j}=\frac{J}{2}\left[\left(S_{i}^{+}\right)_{\alpha \beta}\left(S_{j}^{-}\right)_{\gamma \delta}+\left(S_{i}^{-}\right)_{\alpha \beta}\left(S_{j}^{+}\right)_{\gamma \delta}\right]-K\left(S_{i}^{z}\right)_{\alpha \beta}\left(S_{j}^{z}\right)_{\gamma \delta}
$$

An obvious symmetry of $W_{\alpha \beta, \gamma \delta}^{i j}$, which we use, is $W_{\alpha \alpha^{\prime}, \beta \beta^{\prime}}^{i j}=W_{\alpha \alpha^{\prime}, \beta \beta^{\prime}}^{j i}$. The equation of motion is, therefore

$$
i \partial G_{\alpha \alpha^{\prime}, \beta \beta^{\prime}}^{i j}\left(t-t^{\prime}\right) / \partial t=\frac{1}{2 \pi} \delta\left(t-t^{\prime}\right)<\left[\hat{P}_{\alpha \alpha^{\prime}}^{i}, \hat{P}_{\beta \beta^{\prime}}^{j}\right]_{-}>+<<\left[\hat{P}_{\alpha \alpha^{\prime}}^{i}, H\right]_{-} \mid \hat{P}_{\beta \beta^{\prime}}^{j}>>
$$

If we substitute Eq. (C5) in Eq. (C7), rearrange dummy indices , and use Eq.(C1) and the symmetry of $W_{\alpha \alpha^{\prime}, \beta \beta^{\prime}}^{i j}$, we obtain the following equation of motion for the Fourier transform (i.e., in $\mathbf{q}, \omega$-space) of the Green function.

$$
\begin{gathered}
\omega G_{\alpha \alpha^{\prime}, \beta \beta^{\prime}}^{i j}(\mathbf{q}, \omega)=\frac{1}{2 \pi}<\delta_{i j}\left(\delta_{\alpha \beta^{\prime}} \hat{P}_{\alpha \beta^{\prime}}^{i}-\delta_{\beta \alpha^{\prime}} \hat{P}_{\beta \alpha^{\prime}}^{i}\right)> \\
-\sum_{\mu \mu^{\prime}}<u_{\mu \mu^{\prime}}^{i}\left(\hat{P}_{\alpha \mu^{\prime}}^{i} \delta_{\alpha^{\prime} \mu}-\hat{P}_{\mu \alpha^{\prime}}^{i} \delta_{\alpha \mu^{\prime}}\right) \hat{P}_{\beta \beta^{\prime}}^{j}>-\sum_{k, \mu \nu \nu^{\prime}} W_{\alpha^{\prime} \mu, \nu \nu^{\prime}}^{i k}<<\hat{P}_{\alpha \mu}^{i} \hat{P}_{\nu \nu^{\prime}}^{k} \mid \hat{P}_{\beta \beta^{\prime}}^{j}>> \\
+\sum_{k, \mu \nu \nu^{\prime}} W_{\mu \alpha, \nu \nu^{\prime}}^{i k}<<\hat{P}_{\mu \alpha^{\prime}}^{i} \hat{P}_{\nu \nu^{\prime}}^{k} \mid \hat{P}_{\beta \beta^{\prime}}^{j}>>.
\end{gathered}
$$

In terms of the Hubbard operators, the RPA is defined by the decoupling in which a three-operator Green function is factorised into products of two-operator Green functions and expectation values of the remaining operator, as shown below: 


$$
\begin{aligned}
<<\hat{P}_{\alpha \alpha^{\prime}}^{m} \hat{P}_{\gamma \gamma^{\prime}}^{l} \mid \hat{P}_{\beta \beta^{\prime}}^{n}>>_{\omega}=\delta_{\alpha \alpha^{\prime}}<\hat{P}_{\alpha \alpha^{\prime}}^{m}>G_{\gamma \gamma^{\prime}, \beta \beta^{\prime}}^{l n}(\omega)+\delta_{\gamma \gamma^{\prime}}<\hat{P}_{\gamma \gamma^{\prime}}^{l}>G_{\alpha \alpha^{\prime}, \beta \beta^{\prime}}^{m n}(\omega) \\
+\delta_{l m}\left[\delta_{\alpha^{\prime} \gamma} G_{\alpha \gamma^{\prime}, \beta \beta^{\prime}}^{m n}(\omega)-\delta_{\gamma^{\prime} \alpha} G_{\gamma \alpha^{\prime}, \beta \beta^{\prime}}^{m n}(\omega)\right]
\end{aligned}
$$

In our model (C5), the last term on the right hand side of Eq.(C9), arising out of the commutation of $\hat{P}_{\alpha \alpha^{\prime}}^{m}$ and $\hat{P}_{\gamma \gamma^{\prime}}^{l}$, vanishes as there is no interaction connecting the sites on the same sublattice (the delta-function $\delta_{l m}$ requires this but $W_{\alpha \alpha^{\prime}, \beta \beta^{\prime}}^{i j}=0$ for $i=j$ ). In a model with interactions connecting the same sublattices (e.g., a model with further than nearest-neighbour interactions) this term has to be considered.

By using the RPA factorisation in Eq.(C8) we obtain

$$
\left(\omega-\varepsilon_{\alpha \alpha^{\prime}}^{i}\right) G_{\alpha \alpha^{\prime}, \beta \beta^{\prime}}^{i j}(\mathbf{q}, \omega)=L_{\alpha^{\prime} \alpha}^{i} \sum_{k, \mu \nu} W_{\alpha \alpha^{\prime}, \nu \mu}^{i k} G_{\mu \nu, \beta \beta^{\prime}}^{k j}(\mathbf{q}, \omega)+\frac{1}{2 \pi} L_{\alpha \alpha^{\prime}}^{i} \delta_{i j} \delta_{\alpha \beta^{\prime}} \delta_{\beta \alpha^{\prime}}
$$

where

$$
\begin{gathered}
\epsilon_{\alpha}^{i}=u_{\alpha \alpha}^{i}+\sum_{l, \gamma} W_{\alpha \alpha, \gamma \gamma}^{i l}(\mathbf{q}=0)<\hat{P}_{\gamma \gamma^{\prime}}^{l}>, \\
\varepsilon_{\alpha \alpha^{\prime}}^{i}=\epsilon_{\alpha}^{i}-\epsilon_{\alpha^{\prime}}^{i},
\end{gathered}
$$

and

$$
L_{\alpha \alpha^{\prime}}^{m}=<\hat{P}_{\alpha \alpha}^{m}>-<\hat{P}_{\alpha^{\prime} \alpha^{\prime}}^{m}>
$$

Since the site indices can have 2 values (denoting the 2 sublattices), and since $\alpha$ and $\alpha^{\prime}$ can have only two values, the most general form of the above equations of motion here is a $4 \times 4$ matrix equation given by 


$$
\begin{gathered}
\left(\begin{array}{cccc}
\omega-2 R_{B} & 0 & \Pi_{+\mathbf{q}}^{A} & \Pi_{-\mathbf{q}}^{A} \\
0 & \omega+2 R_{B} & -\Pi_{-\mathbf{q}}^{A} & -\Pi_{+\mathbf{q}}^{A} \\
\Pi_{+\mathbf{q}}^{B} & \Pi_{-\mathbf{q}}^{A} & \omega-2 R_{A} & 0 \\
-\Pi_{-\mathbf{q}}^{A} & -\Pi_{-\mathbf{q}}^{A} & 0 & \omega+2 R_{A}
\end{array}\right)\left(\begin{array}{c}
G_{+-\beta \beta^{\prime}}^{A B}(\mathbf{q}, \omega) \\
G_{-+\beta \beta^{\prime}}^{A B}(\mathbf{q}, \omega) \\
G_{+-\beta \beta^{\prime}}^{B B}(\mathbf{q}, \omega) \\
G_{-+\beta \beta^{\prime}}^{B B}(\mathbf{q}, \omega)
\end{array}\right) \\
=\frac{1}{2 \pi} L_{+-}^{B}\left(\begin{array}{c}
0 \\
0 \\
\delta_{+\beta^{\prime}} \delta_{\beta-} \\
-\delta_{-\beta^{\prime}} \delta_{\beta+}
\end{array}\right),
\end{gathered}
$$

where $\beta \beta^{\prime}$ take values +- or -+ and

$$
\Pi_{ \pm \mathbf{q}}^{i}=\frac{1}{2}\left[ \pm J(\mathbf{q})\left(1+\cos \theta_{A} \cos \theta_{B}\right)-K(\mathbf{q}) \sin \theta_{A} \sin \theta_{B}\right] L_{+-}^{i} .
$$

The sublattice indices can be dropped in the superconducting and nonordered phases (i.e., there is no broken lattice-translational symmetry). This matrix equation (C14) reduces to a $2 \times 2$ matrix equation in the CDW and the superconducting phases and to a scalar equation for the nonordered metallic phase because of the symmetry of these phases.

\section{Appendix D}

\section{Scaling forms for Thermodynamic Functions near Multicritical Points}

In the phase diagrams of Figs.III.1 and III.2 the following multicritical points occur: bicritical points, tricritical points, tetracritical points and critical end points. For the sake

of completeness we give the scaling form [111] for the singular part of the free energy in the vicinities of these points.

\section{Bicritical and Tetracritical Points}

Bicritical and tetracritical points are studied easily using the same model, namely, the Landau-Ginzburg free-energy functional [98, 99, 112]. 


$$
\mathcal{F}_{L G}=\int d^{d} x\left[\frac{1}{2}\left((\nabla \psi)^{2}+r_{0}|\psi|^{2}\right)+u|\psi|^{4}-\frac{1}{2} g\left(\psi_{1}^{2}-\frac{1}{2}\left(\psi_{2}^{2}+\psi_{3}^{2}\right)\right)+v \sum_{i=1}^{3} \psi_{i}^{4}\right],
$$

where $\psi$ is a three-component, vector order parameter with components $\psi_{i}, i=1 \ldots 3$. A bicritical point is obtained if $v<0$ and a tetracritical point if $v>0$. The temperature-like scaling variable $t$ depends linearly on the deviation of $r_{0}$ from its value at the multicritical point.

The singular part of the free energy $\mathcal{F}_{s}$ assumes the following scaling form in the vicinity of a bicritical point which occurs at the temperature $T_{b}$ :

$$
\mathcal{F}_{s}=\left(T-T_{b}\right)^{2-\alpha_{b}} \mathcal{F}_{b}\left(\frac{g}{\left(T-T_{b}\right)^{\phi_{g}}}, \frac{v}{\left(T-T_{b}\right)^{\phi_{v}}}\right),
$$

where the mean-field values of the exponents are $\alpha_{b}=0, \phi_{g}=1$ and $\phi_{v}=0$. The specificheat exponent $\alpha_{b}$ is related to the correlation length exponent $\nu_{b}$ via the hyperscaling relation $d \nu_{b}=2-\alpha_{b}$, where $d$ is the dimension ( the mean-field value of $\nu_{b}=1 / 2$ ). To leading order in $\epsilon=4-d$ the bicritical exponents are

$$
\begin{gathered}
\nu_{b}=1 / 2(1+5 \epsilon / 22), \\
\phi_{g}=1+3 \epsilon / 22, \\
\phi_{v}=-\epsilon / 22 .
\end{gathered}
$$

Note that, even though $v$ is irrelevant, it should be displayed explicitly since it is a dangerous irrelevant variable. More details regarding this point and a comprehensive discussion of the (similar) scaling in the vicinity of tetracritical points can be found elsewhere [98, 111, 113.

For the anisotropic antiferromagnets, that we are interested in, the Landau-Ginzburg free energy is an expansion in powers of $\mathbf{M}$ and $\mathbf{M}_{s}$, the magnetization and the staggered 
magnetization. However, in the vicinity of the bicritical point, such a free energy can always be put in the form $(\mathrm{C} 1)$ given above.

\section{Tricritical Point}

In the vicinity of a tricritical point the Landau-Ginzburg free-energy functional can be written as [113, 114, 115, 116, 117]

$$
\mathcal{F}_{L G}=\int d^{d} x\left[\frac{1}{2}\left((\nabla \psi)^{2}+a_{2} \psi^{2}\right)+a_{4} \psi^{4}+\frac{1}{6} a_{6} \psi^{6}-H \psi\right]
$$

where $\psi$ is a scalar order parameter. The tricritical point occurs at $H=0, a_{2}=a_{4}=0$, and $a_{6}>0$ ( $a_{2}$ depends linearly on temperature $)$. The scaling form for the singular part of the free energy $\mathcal{F}_{s}$ has the following form in the vicinity of the tricritical point $T_{t}$ :

$$
\mathcal{F}_{s}=\left(T-T_{t}\right)^{2-\alpha_{t}} \mathcal{F}_{t}\left(\frac{g}{\left(T-T_{t}\right)^{\phi_{t}}}, \frac{H}{\left(T-T_{t}\right)^{\phi_{v}}}\right)
$$

where, in mean-field theory, the exponents are $\alpha_{t}=1 / 2, \phi_{t}=1 / 2, \Delta_{t}=5 / 4$, and the scaling field $g$ is defined to be proportional to $a_{4}$. Since the upper-critical dimension for tricritical behaviour is 3, corrections to mean-field theory are weak logarithmic ones 111, 113.

\section{Critical End Point}

The simplest Landau-Ginzburg theory which yields a critical end point is defined by the functional [111, 118, 119, 120

$$
\mathcal{F}_{L G}=\int d^{d} x\left[\frac{1}{2}\left((\nabla \psi)^{2}+r \psi^{2}\right)+u \psi^{4}+v \psi^{6}+w \psi^{8}\right]
$$

with $v<0, w>0$, and $\psi$ a scalar order parameter. (For the critical end point shown in Fig.III.2 a more complicated Landau-Ginzburg functional would be required, but Eq.(D6) suffices for the purpose of illustrating the essential features associated with 
this point.) If $\psi$ is uniform and we use mean-field theory (i.e., we minimise $\mathcal{F}_{L G}$ with

respect to $\psi$ ), then a critical end point is encountered at $u=\frac{v^{2}}{4 w}$ and $r=0$. As this point is approached from the disordered phase, thermodynamic functions, such as the orderparameter susceptibility $\chi$, diverge as they do along the critical line which terminates at the critical end point (Fig.III.2); i.e., at the mean-field level $\chi S_{i}^{-} r^{-1}$ as $r \rightarrow 0$ (as usual r depends linearly on the deviation of the temperature from the temperature at which the critical end point occurs). However, the order parameter jumps from zero to $(|v| / 2 w)^{1 / 2}$ as we go from the disordered phase to the ordered phase through the critical end point. This behavior does not depend on the mean-field approximation. Even renormalizationgroup calculations [118, 119, 120] yield the same qualitative behavior: Thermodynamic functions like $\chi$ diverge with the same exponent at a critical end point as they do along the critical line which terminates at this point and the order parameter jumps at the critical end point.

\section{Appendix E}

\section{The Current Operator in the Pseudospin Model}

The effective Hamiltonian in the pseudospin model is (see Appendix A)

$$
\begin{aligned}
H=4 \frac{|t|^{2}}{U} \sum_{<i j>} S_{i}^{z} \cdot S_{j}^{z}+ & 4 \frac{t^{2}}{U} \sum_{<i j>}\left(S_{i}^{+} S_{j}^{-}+H . C .\right)+4 V \sum_{<i j>} S_{i}^{z} \cdot S_{j}^{z} \\
& -B \sum_{i}\left(2 S_{i}^{z}+1\right) .
\end{aligned}
$$

To couple this to an electromagnetic field we use the Peierls substitution $t \rightarrow$ $t \exp -i \int_{i}^{j} \mathbf{A} . d \mathbf{l}$ where $\mathbf{A}$ is the vector potential $(\hbar=c=1)$. Such a substitution will obviously leave the first term in $\mathrm{H}$ unaffected and the only contribution to the current comes from the transverse part of the pseudospin-pseudospin interaction term.

By expanding $H$ to linear order in $\mathbf{A}$, and noting that a minimal coupling to the 
current is of the form $\mathbf{j}$.A, we can easily identify the current operator for the above model as

$$
\mathbf{j}=-i(2 e) \sum_{<i j>} J \mathbf{a}\left(S_{i}^{+} \cdot S_{j}^{-}-H . C .\right),
$$

where $\mathbf{a}$ is the nearest-neighbour vector and $J=4 \frac{|t|^{2}}{U}$.

There are alternative ways of deriving the current operator (of course with identical results) for the projected Hamiltonian . For example, we can construct the dipole operator $\mathbf{P}$ for this model and then use the relation $\mathbf{j}=-i[\mathbf{P}, H]_{-}$to get the current operator.

\section{Appendix F}

The Ginzburg-Landau Equation and the Superconducting Coherence Length for the Pseudospin Model

The Ginzburg-Landau free-energy functional for the pseudospin model in terms of the order parameters $<S_{i}^{+}>=\psi_{i}$ and $2<S_{i}^{z}>=(n-1)=m$ is:

$$
\begin{gathered}
\mathcal{F}[\psi]=-J / N \sum_{<i j>}\left(\psi_{i}^{\dagger} \psi_{j}+H . C .\right)+K_{0} m^{2} \\
+(\beta N)^{-1} \sum_{i}\left[\left(S_{i}-1 / 2\right) \log \left(S_{i}-1 / 2\right)+\left(S_{i}+1 / 2\right) \log \left(S_{i}+1 / 2\right)\right],
\end{gathered}
$$

where $\mathrm{N}$ is the number of sites, and

$$
S_{i}^{2}=\left|\psi_{i}\right|^{2}+m^{2} / 4
$$

It is then easy to show that an expansion to order $\psi^{2}$ gives the phase boundary between the superconducting and nonordered phases. To this order the free energy per site is

$$
\mathcal{F}[\psi]=-J / N \sum_{<i j>}\left(\psi_{i}^{\dagger} \psi_{j}+H . C .\right)+(\beta N)^{-1} \sum_{i}\left|\psi_{i}\right|^{2} /(m \log n /(1-m)) .
$$


which of course gives the same expression for $T_{C N}$ as Eq.(B8)

Note that the coupling to the electromagnetic field is expressed in terms of the following gauge transformation on the bond $(i j)$,

$$
\psi_{i} \psi_{j} \rightarrow \psi_{i} \psi_{j} \exp -i(2 e) \int_{i}^{j} \mathbf{A} \cdot \mathbf{d l} .
$$

where $\mathbf{A}$ is the vector potential. Note that the current operator (Appendix E) follows from a functional derivative of $\mathcal{F}$ with respect to $\mathbf{A}$

To calculate the coherence length, we take the magnetic field $H$ to be in the $z$ direction, so that, in the Landau gauge, the vector potential is $\mathbf{A}=(0,-H x, 0)$. Then

$$
\psi_{l} \psi_{m} \rightarrow \psi_{l} \psi_{m} \exp \left[-i \phi_{l m}\right]
$$

where

$$
\phi_{l m}=2 e H l_{x} a^{2}
$$

Here $a$ is the lattice constant and $l_{x}$ is the $x$-coordinate of the $l$-th site;

Close to the transition, by expanding the Ginzburg-Landau free energy and retaining terms upto order of $\psi^{2}$, we obtain

$$
\mathcal{F}[\psi]=-J / N \sum_{<i j>}\left(\psi_{i}^{\dagger} \psi_{j}+H . C .\right)+T / T_{S N}(0) \sum_{i}\left|\psi_{i}\right|^{2}
$$

where $\mathrm{T}_{S N}(\mathrm{H})$ is the transition temperature in a magnetic field $\mathrm{H}$.

An expansion of the first term on the right hand side gives the $(\nabla \psi)^{2}$ term. By equating the coefficient of this term to $\xi^{2}$ (where $\xi$ is the superconducting coherence length) we obtain

$$
\xi^{2}=a^{2} / \tau,
$$


where $\tau=1-T / T_{S N}(0)$. (Equivalently, one can write down the equation for $\psi_{i}$ and compare it with the eigenvalue equation of a quantum oscillator.)

Hence, as we have already mentioned in the text, the coherence length well away from the transition is about one a lattice constant. This result is meaningful from a physical point of view as, in the large- $U$ theory, there is no length scale in the problem other than the lattice constant. 


\section{References}

[1] A. W. Sleight, J. L. Gillson and P. E. Bierstedt, Solid State Comm., 17, 27 (1975); A. W. Sleight, J. L. Gillson and P. E. Bierstedt, Solid State Comm., 88, $841(1993)$.

[2] S. Uchida, K. Kitazawa and S. Tanaka, Phase Trans.,8, 95 (1987).

[3] L. F. Mattheiss and D. R. Hamman, Phys. Rev. Lett.60, 2681 (1988).

[4] L. F. Mattheiss, E. M. Gyorgy and D. W. Jonston Jr., Phys. Rev. B 37, 3745 (1988).

[5] R. J. Cava, B. Batlogg, J.J. Krajewski, R. Farrow, L.W. Rupp Jr., A.E. White, K. Short, W.F. Peck, and T. Kometani, Nature, 332, 814 (1988).

[6] R. J. Cava, B. Batlogg, G. P. Espinoza, A. P. Ramirez, J. J. Kajewski, W. F. Peck Jr., L. W. Rupp Jr. and A. S. Cooper, Nature, 339, L291 (1989).

[7] R. J. Cava anb B. Batlogg, MRS Bulletin, 49, (Jan. 1989).

[8] S. Pei, N. J. Zalusec, J. D. Jonston, D. G. Hinks, A. W. Mitchell and D. R. Richards, Phys. Rev. B 39, 811 (1989); S. Pei, et al., ibid, 41, 4126 (1990).

[9] A. Taraphder, Ph.D. Thesis, Indian Institute of Science (1991), unpublished.

[10] A. Taraphder, H.R. Krishnamurthy, R. Pandit, and T. V. Ramakrishnan, Europhys. Lett. 21, 79 (1993); A. Taraphder, H. R. Krishnamurthy, R Pandit, and T. V. Ramakrishnan, Phys. Rev. B 521368 (1995).

[11] A. Taraphder, H. R. Krishnamurthy, R. Pandit, and T. V. Ramakrishnan, (1995b, to be published).

[12] M. B. Robin and P. Day, Adv. Inorg. Chem. Radiochem., 10, 247 (1967). 
[13] N. Jones, J. Parise, R. Flippen and, A. W. Sleight, Jl. Solid State Chem.(Lett),38, 319 (1989).

[14] D. G. Hinks, B. Dabrowski, J.D. Jorgensen, A.W. Mitchell, D.R. Richards, S. Pei, and Donglu Shi, Nature,333, 836 (1988);

[15] D.G. Hinks, D.R. Richards, B. Dabrowski, D. T. Marx, and A.W. Mitchell, Nature 335, 419 (1988).

[16] Y. Koyama and M. Ihimara Phys. Rev. B, 45, 9966 (1992).

[17] H. Minami, Physica C, 185-187, 1257 (1991).

[18] C. M. Varma, Phys. Rev. Lett.61, 2713 (1989)

[19] A. W. Sleight, in Chemistry of Oxide Superconductors, ed. C. N. R. Rao, (Blackwell Scientific Publications, Oxford, 1988), p. 27.

[20] R. Seshadri, V, Manivannan, K.P. Rajeev, J. Gopalakrishnan, and C.N.R. Rao, Jl. Solid State Chem.(Lett.), 89 (1990).

[21] B. Batlogg, R. J. Cava, L. W. Rupp Jr., J. J. Kajewski and H. F. Peck Jr., Bell Laboratory-preprint (1990)), to appear in The Proceedings of The International Winter School on Electronic Properties of HTSC. and Related Materials, Austria.

[22] Y. J. Uemura, B. J. Sternlib, D. E. Cox, J. W. Brewer, R. Kadano, J. R. Kempton, R. E. Keifl, S. R. Kreitzman, G. M. Luke, P. Mulhern, T. Riseman, D. L. Williams, W. J. Kossler, X. H. Yu, C. E. Stronach, M. A. Subramanian, J. Gopalakrishnan and A. W. Sleight, Nature,335, 151 (1988). 
[23] B. Batlogg, J. P. Remeika, R. C. Dynes, H. Barz, A. S. Cooper and J. P. Garno, Superconductivity in $d$ - and $f$ - Band Metals (Kenforschungszentrum Karlsruhe), N. Buckel and W. Weber, eds. (1982) p 401.

[24] B. Batlogg, R. J. Cava, L. W. Rupp Jr., A. M. Mujsee, J. J. Kajewski, J. P. Remeika, W. F. Peck Jr., A., S. Cooper, G. P. Espinoza, Phys. Rev. Lett.61,1670 (1988);

[25] K. Machida, Physica C, 156, 276 (1988).

[26] D. E. Cox and A. W. Sleight, Solid State Comm., (19, 969 (1976); Acta Crystallographica, Sec.B 35, 1 (1979).

[27] G.U. Kulkarni, V. Vijayakrishnan, G. Ranga Rao, R. Seshadri, and C.N.R. Rao, Appl. Phys. Lett,57, 1823 (1990).

[28] S. Sugai, S. Uchida, K. Kitazawa, S. Tanaka and a. Katsui, Phys. Rev. Lett.55, 426 (1985); Jpn. Jl. Appl. Phys., 24, suppl 24-2, 13(1985).

[29] S. Pei, J. D. Jorgensen, D.G. Hinks, B. Dabrowski, D.R. Richards, A.W. Mitchell, Y. Zheng, J.M. Newsam, S. K. Sinha, D. Vaknin, and A. J. Jacobson, Physica C,162-164, 556 (1989); S. Pei, J. D. Jorgensen, B. Dabrowski, D. G. Hinks, D.R. Richards, A.W. Mitchell, J.M. Newsam, S. K. Sinha, D. Vaknin, and A. J. Jacobson, Phys. Rev. B 41, 4126 (1990); J. Marcus et al., Solid State Comm. 78, 967 (1991); D. T. Marx et al., Phys. Rev. B 46, 1144 (1993).

[30] B. Batlogg, R. J. Cava, L. W. Rupp Jr., G. P. Espinosa, J. J. Krajewski, W. F. Peck Jr., and A. S. Cooper, Physica C 162-164, 1393 (1989a).

[31] B. Batlogg, R. J. Cava, L. F. Schneemeyer, and G. P. Espinosa, IBM Journal of Res. \& Develop. 33, 208 (1989b). 
[32] B. Batlogg, Physica B 126, 275(1984)

[33] M. Licheron and F. Gervais, Phys. Rev. B 47, 8008 (1993).

[34] S. Kondoh, M. Sera, M. Fukuda, Y. Ando and M. Sato, Solid State Commun.67, 879 (1989).

[35] S. Kondoh, M. Sera, Y. Ando, and M. Sato, Physica C157, 469 (1989).

[36] K. Kitazawa, S. Uchida, and K. Tanaka, Physica B135, 505 (1985).

[37] K. Kitazawa, A. Katsui, A. Toriumi and, S. Tanaka, Solid State Comm. 52, 459 (1984).

[38] T. Thanh, A. Koma and S. Tanaka, Appl. Phys.22, 205 (1980); D. P. Moiseev, S. Uranova and M. Fenik, Sov. Phys. Solid State bf 23, 1371 (1981), R. A. Schweinfurth, et al., Appl. Phys. Lett. 61,(4), 480 (1992).

[39] T. Tani, T. Itoh and S. Tanaka, Jl. Phys. Soc. Jpn. 49, Suppl 4, 309 (1980).

[40] J. E. Graebner, L. F. Schneemeyer and J. K. Thomas, Phys. Rev. B 39, 9682 (1989).

[41] M. Oda, Y. Hidaka, A. Katsui and T. Murakami, Jpn. Jl. Appl. Phys., 24, suppl 24-2, 314 (1985).

[42] T. Itoh, K. Kitazawa and S. Tanaka, Jl. Phys. Soc. Jpn.,53, 2668 (1984).

[43] S. Tajima, K. Kitazawa and S. Tanaka, Solid State Comm.,47,659 (1983).

[44] L. F. Mattheiss and D. R. Hamman, Phys. Rev. B28, 4227 (1983); Phys. Rev. B 26, 2686 (1982). 
[45] L. F. Mattheiss, in Oxygen Disorder Effects in High-Tc Superconductors, edited by J. L. Moran-Lopez and I. K. Schuller (Plenum, New York, 1990), p.117.

[46] A.I. Liechtenstein, I.I. Mazin, C.O. Rodriguez, O. Jepsen, O.K. Andersen, and M. Methfessel, Phys. Rev. B 44, 5388 (1991). The LDA ground state calculation of Liechtenstein et al., is indifferent to whether the Bi-6s orbital occupancies can be described in terms of a negative $U$ or a positive $U$. The situation is somewhat similar to LDA calculations of $\mathrm{La}_{2} \mathrm{CuO}_{4}$ [121, 122], which give a qualitatively incorrect ground state. However, the existence of a large positive $U$ in this system was inferred by Hybertsen, Schluter and Christensen [121] by doing an LDA calculation of energy as a function of locally constrained d-electron number [123]. Similar calculations have not been carried out for $\mathrm{BaBiO}_{3}$ and its derivatives. Therefore, the fact that an LDA calculation for $\mathrm{Ba}_{1-x} \mathrm{~K}_{x} \mathrm{BiO}_{3}$ leads to a small-gap semiconducting ground state with a structural distortion (at the correct value of $x$, but in a rigid-band approximation) but with no charge disproportionation, is not argument against the existence of a local negative U. Further, the electronic-structure results are inconsistent with the observed eletrical and optical gaps as we discuss in the text.

[47] S. H. Blanton et al., Phys. Rev. B 47, 996 (1993).

[48] H. Sato et al., Phys. Rev. B 48, 6617 (1993).

[49] S. Tajima, S. Uchida, A. Masaki, H. Takagi, K. Kitazawa, S. Tanaka, and S. Sugai, Phys. Rev. B 35, 696 (1987).

[50] E. S. Hellman, B. Miller, J. M. Rosamilia, E. H. Hartford and K. W. Baldwin, Phys. Rev. B 44, 9719 (1991). 
[51] J. Marcus et al., Solid State Comm., 78, 967 (1991); M. Affronte, J. Marcus and C. Escribe-Filippini, Solid State Comm., 78, 501 (1993).

[52] B. Dabrowski, D. G. Hinks, J. D. Jorgensen, R. K. Kalia, P. Vashista, D. R. Richards, D. T. Marx and A. W. Mitchell, Physica C 156, 24 (1988).

[53] I. Bozovic et al., Phys. Rev. B 46, 1182 (1992).

[54] S. Uchida, S. Tajima, A. Masaki, S. Sugai, K. Kitazawa and S. Tanaka, Jl. Phys. Soc. Jpn., 54, 4895 (1985).

[55] S. Tajima, S. Uchida, A. Masaki, H. Takagi, K. Kitazawa, S. Tanaka and A. Katsui, Phys. Rev. B 32, 6302 (1985).

[56] S. Sugai, Solid State Commun., 72, 1187 (1989).

[57] C. K. Loong, P. Vashishta, R. K. Kalia, M. H. Degani, B. Dabrowski, A. W. Mitchell, D. R. Richards and Y. Zheng, Phys. Rev. Lett.62, 2628 (1989).

[58] D. G. Hinks MRS Bulletin 15, 65 (1990).

[59] J. O. Sofo, A. A. Alizia, and M. D. Nunez Regueiro, Phys. Rev. B 39, 9701 (1989).

[60] J.F. Federici, B.I. Greene, E.H. Hartford, and E.S. Hellman, Phys. Rev. B 42, 923 (1990).

[61] G. Ruani, A.J. Pal, C. Taliani, R. Zamboni, X. Wei, L. Chen, and Z.V. Vardeny, Synthetic Metals, 41-43, 3977 (1991).

[62] M. Shirai, N. Suzuki, and M. Motizuki, J. Phys. Cond. Matter 1, 2939 (1989).

[63] Y. Enomoto, M. Oda and T. Murakami, Phase Transition 8, 129 (1987).

[64] J. M. Valles, Jr. and R. C. Dynes, Mater. Res. Soc. Bulletin 15, 51 (1990). 
[65] Z. Schlesinger, et al., Phys. Rev. B 40, 6862 (1989); Z. Schlesinger and R. T. Collins, Mater. Res. Soc. Bulletin 15, 44 (1990); T. Hasegawa, H. Ikuta and K. Kitazawa, in Physical Properties of High Temperature Superconductors , vol.III, ed. D. M. Ginsberg, World Scientific (1992), p. 525.

[66] G. Crabtree, Jl. Magn. Matl., 76-77, 547 (1988); C. Escribe-Filippini, et al., private communication and to be published.

[67] Q. Huang et al., Nature 347, 369 (1990).

[68] E. S. Hellman et al., Appl. Phys. Lett. 55, 2120 (1989).

[69] B. M. Moon et al., Appl. Phys. Lett. 59, 1905 (1991).

[70] U. Welp et al., Physica C156, 27 (1988).

[71] W. K. Kwok et al., Phys. Rev. B 40, 9400 (1989).

[72] F. Sharifi et al., Phys. Rev. B 44, 12522 (1991).

[73] S. Martin et al., Phys. Rev. B 48, 6626 (1993).

[74] M. Suzuki and T. Murakami, Solid State Commun. 53, 691 (1985).

[75] C. Chaillout, J.P. Remeika, A. Santoro, and M. Marezio, Solid State Commun. 56, 829 (1985).

[76] C. Chaillout, A. Santoro, J.P. Remeika, A.S. Cooper, G.P. Espinosa, and M. Marezio, Solid State Commun.65, 1363 (1988).

[77] W. D. Mosley et al., Phys. Rev. B 48, 611 (1993).

[78] T. M. Rice and L. Sneddon, Phys. Rev. Lett.47, 689 (1981).

[79] E. Jurczek and T. M. Rice, Europhys. Lett.1, 225 (1986). 
[80] R. Micnas, J. Ranninger and S. Robaszkiewicz, Rev. Mod. Phys.62, 113(1990).

[81] A. Taraphder and B. K. Chakraverty, Physica C 235-240, 2351 (1994).

[82] M. J. Rice and Y. Wang, Physica C 157, 192 (1989).

[83] E. Jurczek, Phys. Rev. B 32, 4708 (1985).

[84] A. Taraphder and P. Coleman, Phys. Rev. Lett. 66, 2814 (1991).

[85] B. K. Chakraverty and J. Ranninger, Phil. Mag.52, 669 (1985).

[86] S. Robaszkiewicz, R. Micnas and K. Chao, Phys. Rev.B 23, 1447 (1981); Phys. Rev. B 24, 1579 (1981).

[87] P. W. Anderson, Phys. Rev. Lett. 34, 953 (1975).

[88] M. Blume, V. Emery and R. B. Griffiths, Phys. Rev. A4, 1071 (1971).

[89] D. Yoshioka and H. Fukuyama, J. Phys. Soc. Jpn., 54, 2996 (1985).

[90] A. Alexandrov and J Ranninger, Phys. Rev. B 23, 1796 (1981).

[91] D. Gaitonde and S. N. Behera, Physica C 173, 293 (1991).

[92] D. Nguyen Manh, D. Mayou anf F. Cyrot-Lackmann, Solid State Comm. 79, 723 (1991); Europhys. Lett. 13167 (1990).

[93] G. Baskaran, in "Fifty years of Landau Theory", Indian Institute of Science, Bangalore (1990), unpublished.

[94] W. Jin, et al., Phys. Rev. B 45, 5535 (1992).

[95] K. S. Liu and M. E. Fisher, Jl. of Low Temp. Phys.10, 655 (1973).

[96] H. Matsuda and T. Tsuneto, Prog. Theor. Phys. Suppl 46, 411 (1970). 
[97] M. Girardeau and R. M. Mazo, Adv. Chem. Phys.24, 187 (1973); M. Girardeau, Phys. Rev. A 42, 3303 (1990).

[98] D. R. Nelson and M. E. Fisher, Phys. Rev. Lett.32, 1350 (1974).

[99] A.D. Bruce and A.A. Aharony Phys. Rev. B 11, 478 (1975).

[100] S.B. Halley and P. Erdös Phys. Rev. B 5, 1106 (1972); J. Hubbard, Proc. Roy. Soc. Lond. Ser. A 285, 542 (1965).

[101] S. Robaszkiewicz, R. Micnas and K. Chao, Phys. Rev. B 26, 3915 (1982).

[102] L. N. Cooper, Phys. Rev. 104, 1189 (1956).

[103] A. B. Migdal, Sov. Phys. JETP, 1, 996 (1958).

[104] C.J. Stanton and J.W. Wilkins, Phys. Rev. B 35, 9722 (1987).

[105] F. J. Ohkawa, Phys. Rev. B 46, 11965 (1993).

[106] S. E. Barnes, J. Phys. F 6, 1375 (1976), ibid 7, 2637 (1977).

[107] J. Bala and M. Oles, Phys. Rev. B 47, 611 (1993).

[108] C. Kittel, Quantum Theory of Solids, McGraw Hill, New York, (1983).

[109] D. Gross, R. Joynt, and T. M. Rice, Phys.Rev. B 36, 281(1987).

[110] D.N. Zubarev, Sov. Phys. Usp. 3, 320 (1960) [Usp. Fiz. Nauk 7, 71 (1960)].

[111] A. A. Aharony, in Critical Phenomena ed. F. J. W. Hahne, Lecture Notes in Physics, Vol.186 Springer, New York, (1983) p 209.

[112] J. M. Kosterlitz, M. E. Fisher and D. R. Nelson, Phys. Rev. B13, 412 (1976);.

[113] M.E. Fisher, in it Critical Phenomena ed. F. J. W. Hahne, Lecture Notes in Physics, Vol.186 ( Springer, New York, (1983 ) p 132. 
[114] D. Mukamel, Phys. Rev. B 14, 1303 (1976).

[115] D.R. Nelson and E. Domany, Phys. Rev. B 13, 236 (1976).

[116] E. Domany, D.R. Nelson, and M.E. Fisher, Phys. Rev. B 15, 3493 (1977);

[117] E. Domany and M.E. Fisher, Phys. Rev. B 15, 3510 (1977).

[118] T.A.L. Ziman, D.J. Amit, G. Grinstein, and C. Jayaprakash, Phys. Rev. B 25, 319 (1982).

[119] J.R. Banavar, D. Jasnow, and D.P. Landau, Phys. Rev. B 20, 3820 (1979).

[120] M. E. Fisher and P. J. Upton, PRL 65, 2402 (1990). 3405(1990).

[121] M. S. Hybertsen, M. Schluter and N. E. Chritensen, Phys. Rev. B 39, 9028 (1989).

[122] A. K. McMahan, R. M. Martin and S. Satpathy, Phys. Rev. B 38, 6650 (1989).

[123] An early calculation of negative $U$ using LDA, but again with a local charge constraint and distortion coordinate as an extra degree of freedom, is that for Si vacancy, due to Baraff, Kane and Schluter [G. A. Baraff, E. O. Kane and M. Schluter, Phys. Rev. B 21, 5662 (1980)].

[124] A. P. Kampf and J. R Schrieffer, Phys. Rev. B 41, 6399 (1990); Phys. Rep. 249, 257 (1995).

Note 1. Some other correlation functions are gapless in the superconducting phase reflecting merely the XY-model nature of the ordering; however, they do not correspond to any physically measurable function.

Note 2. In an earlier treatment [80 it was claimed that a mixed phase with both CDW and superconducting order could be obtained within mean-field theory for model 
by working with fixed $\left\langle S^{z}\right\rangle$ (the analogue of the canonical ensemble). This is not correct. The phase diagrams obtained from both fixed- $B$ (i.e., grand canonical ensemble) and fixed- $<S^{z}>$ (i.e., canonical ensemble) calculations have to be the same by the equivalence of the two ensembles. The first-order boundary of Fig.III.1a (obtained by controlling B) appears as the two-phase coexistence region of Fig.III.1b (obtained by controlling $<S^{z}>$ ); and the discontinuity of $\left\langle S^{z}>\right.$ across the the first-order boundary of Fig.III.1b yields directly the boundaries of the coexistence region of Fig.III.1a. The only difference between the two ensembles is the following: At coexistence and with fixed $<S^{z}>$, the system phase separates into two phases-one superconducting and the other a semiconducting, CDW phase-with one interface between the two. (The fractions of the two phases follow from the lever rule.) However, with fixed $B$ on the first-order boundary), either one or the other phase fills the whole system (since $\left\langle S^{z}\right\rangle$ is not fixed) and there is no interface; formally one or the other can be picked out by allowing $B$ to approach the first-order boundary from either the superconducting or the CDW side. As we have discussed in the text, to obtain a bona fide mixed phase with both supercoducting and charge-density-wave order, next-nearest-neighbour interactions must be added to the pseudospin model. This has also been pointed out recently by ...

Note 3. The bose condensation of the hard-core bosons is observed from the behavior of the chemical potential as one approaches the nonordered--singlet superconductor phase boundary from the high-temperature side. The chemical potential loses explicit dependence on temperature on entering the superconducting phase from the high-temperature disordered phase signifying the onset of a bose condensation of the hard-core bosons. The pseudomagnetic field in the singlet superconducting phase is given by [Eqn (B4)] $B=\left(K_{0}+J_{0}\right)<S^{z}>$ and is clearly independent of temperature.

Note 4. From the width $(\simeq 6 \mathrm{eV})$ of the antibonding band of the LAPW, bandstructure calculation [3, 44]. The value of the hopping matrix element is obtained from 
the experimental values of the (renormalized) DOS at the Fermilevel [24] when fitted to a tight binding band with square density of states, it is less $(\simeq 0.20 \mathrm{eV})$ than the value $(0.3 \mathrm{eV})$ obtained from the band-structure calculation.

Note 5. In earlier work [101 the only a singlet superconducting order parameter $\mathrm{b}_{s}$. In a negative- $U$, extended-Hubbard model-context, choice is naive as we have shown here; both $\mathrm{b}_{s}$ and $\mathrm{b}_{e}$ are nonzero in the superconducting phase.

Note 6: This symmetry is quite easily understood: The negative- $U$ Hubbard model can be transformed into the corresponding positive- $U$ model, as described earlier. (If the chemical potential of the negative- $U$ model is non zero (i.e., the system is away from half filling) this transformation leads to a positive- $U$, Hubbard model with a magnetic field coupling to the spin.) The positive- $U$ Hubbard model has a global $\mathrm{SU}(2)$ spin rotation symmetry that mixes the up and down spins (Affleck, et al. (1988)), whence the $x(y)$ ordered and the $z$ ordered antiferromagnetic (SDW) states are degenerate. In the negative$U$ context, this symmetry is a pseudospin rotation symmetry [84] which means that the superconducting state is degenerate with the CDW state at all filling. [However, as the negative- $U$ model away from half filling maps onto a positive- $U$ Hubbard model with a magnetic field, this symmetry between the $x(y)$ and the $z$-ordered states is lifted, i.e., the corresponding symmetry in the negative- $U$ model, between CDW and superconducting states is destroyed away from half filling. 
Table I.1

Normal State Properties of $\mathrm{BaPb}_{1-x} \mathrm{Bi}_{x} \mathrm{O}_{3}$

\begin{tabular}{|l|r|r|r||l|}
\hline \multicolumn{3}{|c|}{ Metallic } & & Semiconducting \\
\hline$x$ & 0.0 & 0.12 & 0.2 & \multicolumn{1}{c|}{1.0} \\
\hline \hline$\rho(\Omega \mathrm{cm}) \times 10^{-4}$ & 2.10 & 4.15 & 5.40 & \\
\hline$n\left(\mathrm{~cm}^{-3}\right)$ & $1.42 \times 10^{20}$ & $16.0 \times 10^{20}$ & $20.0 \times 10^{20}$ & $\begin{array}{l}\text { Prefactor } 110 \times 10^{20} \\
\text { (activated with gap } \\
\end{array}$ \\
& & & & $0.24 \mathrm{eV})$ \\
\hline$\mu\left(\mathrm{cm}^{2} \mathrm{v}^{-1} \mathrm{~s}^{-1}\right)$ & 210.0 & 9.40 & 5.80 & \\
\hline$\lambda_{\mathrm{mf}}(A)$ & 220.0 & 22.0 & 15.0 & \\
\hline$m^{\star} / m_{e}$ & 0.16 & 0.32 & 0.23 & \\
\hline
\end{tabular}

Table I.2

Superconducting Properties of $\mathrm{BaPb}_{1-x} \mathrm{O}_{3}$ and $\mathrm{Ba}_{1-x} \mathrm{~K}_{x} \mathrm{BiO}_{3}$

\begin{tabular}{|l||c|c|}
\hline & $\mathrm{Ba}_{0.6} \mathrm{~K}_{0.4} \mathrm{BiO}_{3}$ & $\mathrm{BaPb}_{0.75} \mathrm{Bi}_{0.25} \mathrm{O}_{3}$ \\
\hline \hline$d H_{C_{2}} /\left.d T\right|_{T_{c}}$ & $-5 \pm 0.5 \mathrm{KOe} /{ }^{\circ} \mathrm{K}$ & $-5.3 \mathrm{KOe} /{ }^{\circ} \mathrm{K}$ \\
\hline$d H_{C} /\left.d T\right|_{T_{c}}$ & $-75.0 \pm 8.0 \mathrm{Oe} /{ }^{\circ} \mathrm{K}$ & $-50 \pm 5 \mathrm{Oe} /{ }^{\circ} \mathrm{K}$ \\
\hline$-\chi_{\mathrm{GL}}$ & $45.0 \AA$ & $80.0 \AA$ \\
\hline$\xi_{\mathrm{GL}}$ & $70.0 \AA$ & $80.0 \AA$ \\
\hline$\Delta C / T_{c}$ & $2.1 \pm 0.4 \mathrm{~mJ} / \mathrm{moleK}{ }^{2}$ & $0.94 \mathrm{~mJ} / \mathrm{moleK}^{2}$ \\
\hline$N(0)$ & 0.42 states $/ \mathrm{eV}$ & 0.42 states $/ \mathrm{eV}$ \\
$N^{\star}(0)$ & $\leq 0.64$ states $/ \mathrm{eV}$ & $\leq 0.64$ states $/ \mathrm{eV}$ \\
\hline$\lambda_{\text {el-ph }}$ & $\cong 0.5$, (but less than & $\cong 0.7$, (but less than \\
& $0.8)$ & $0.8)$ \\
\hline$\gamma\left(=\Delta C / 1.43 T_{c}\right)$ & $\cong 1.5 \mathrm{~mJ} / \mathrm{moleK}^{2}$ & $0.8 \pm 0.2 \mathrm{~mJ} / \mathrm{moleK}^{2}$ \\
\hline$\lambda_{\text {penetration }}$ & $\cong 4000 \AA$ & $5000-6000 A$ \\
\hline
\end{tabular}


Table II.1

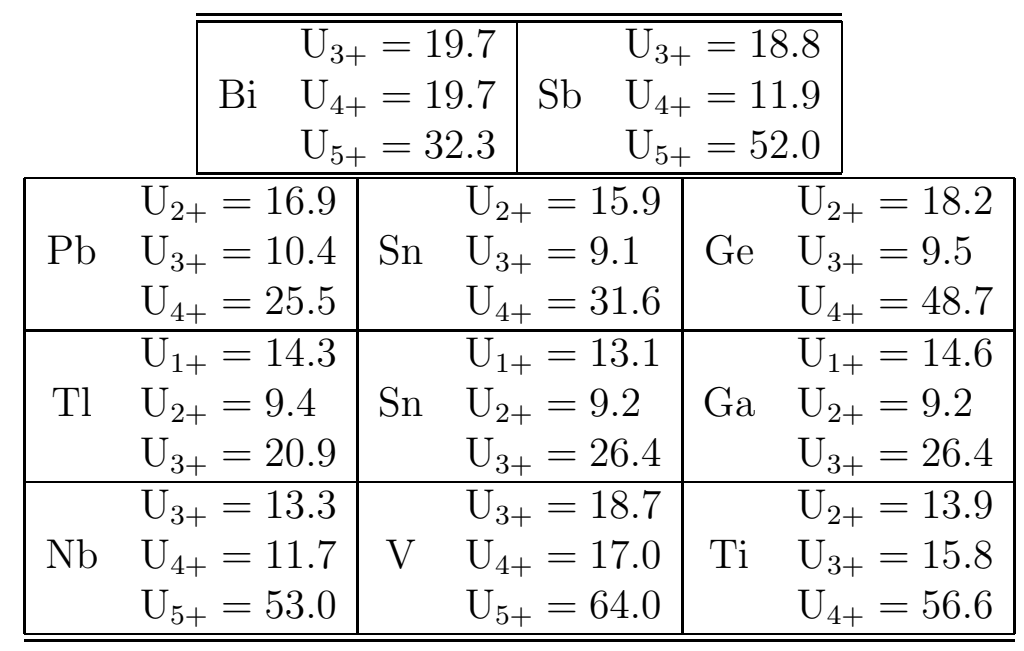

Table II.2

Correspondence between Pseudospin model \& Extended Hubbard model

\begin{tabular}{|l||l|}
\hline Pseudospin model & Extended Hubbard model \\
\hline \hline Up spin & Vacancy \\
\hline Down spin & Double occupancy \\
\hline Magnetisation $m$ & Deviation from $\frac{1}{2}$ filling \\
\hline Magnetic field $B$ & Chemical Potential $\mu$ \\
\hline Antiferromagnetic (AFM) order & $\begin{array}{l}\text { Bose Crystal of Cooperons (CDW } \\
\text { phase) }\end{array}$ \\
\hline $\begin{array}{l}\text { Spin flopped phase (i.e. AFM } \\
\text { with nonzero XY component of } \\
\text { spins) }\end{array}$ & $\begin{array}{l}\text { Bose condensate of Cooperons (S- } \\
\text { wave superconductivity) }\end{array}$ \\
\hline $\begin{array}{l}\text { Non-ordered phase (with partial } \\
\text { alignment for } B \neq 0\end{array}$ & $\begin{array}{l}\text { Bose liquid of the Cooperons } \\
\text { (normal metal) }\end{array}$ \\
\hline
\end{tabular}




\section{Figure Captions}

Fig.I.1a The underlying cubic perovskite structure of $\mathrm{BaBiO}_{3}$ with oxygen atoms at the corners of the octahedra centered around Bi-atoms. Bi-atoms occupy the vertices of a simple-cubic lattice; Ba atoms are at the body centres of this cubic lattice.

Fig.I.1b Possible distortions of this underlying cubic perovskite structure that lead to the various lower-symmetry structures found in $\mathrm{Ba}_{1-x} \mathrm{~K}_{x} \mathrm{BiO}_{3}$ and $\mathrm{BaPb}_{1-x} \mathrm{Bi}_{x} \mathrm{O}_{3}$.

Fig.I.2a Schematic phase diagram of the $\mathrm{BaPb}_{1-x} \mathrm{Bi}_{x} \mathrm{O}_{3}$ system in the $T-x$ plane (after Sleight [1]). The orthorhombic $\left(\mathrm{O}_{I I}\right)$ and tetragonal $(\mathrm{T})$ phases are metallic at high temperatures and superconducting at low temperatures. The orthorhombic $\left(\mathrm{O}_{I I}\right)$ and monoclinic(M) phases are semiconducting. The precise nature of the metalsemiconductor is not clear, but here it is shown as a first-order transition with the hatched region indicating a region of two-phase coexistence. All the four phases undergo high-temperature structural transitions to a cubic phase.

Fig.I.2b Schematic phase diagram of the $\mathrm{Ba}_{1-x} \mathrm{~K}_{x} \mathrm{BiO}_{3}$ system in the $T-x$ plane after Pei, et al. [8, 14] The entire cubic phase is metallic and the hatched, low temperature region is a superconducting phase. The monoclinic $(\mathrm{M})$, orthorhombic $(\mathrm{O})$, and rhombohedral $(\mathrm{R})$ regions are semiconducting.

Fig.I.3a Temperature dependence of the resistivity of $\mathrm{BaPb}_{1-x} \mathrm{Bi}_{x} \mathrm{O}_{3}$ on a log-log scale in the metallic regime and across the metal-semiconductor transition regime. Note that the temperature-coefficient of resistivity is negative at $x=0.3$, but resistivity goes to zero at low temperatures. (Afte Uchida et al. [2])

Fig.I.3b The temperature dependence of the electrical resistivity of $\mathrm{BaPb}_{1-x} \mathrm{Bi}_{x} \mathrm{O}_{3}$ in the semiconducting regime (after Uchida et al. [2]). 
Fig.I.3c Temperature dependence of the electrical resistivity of $\mathrm{Ba}_{1-x} \mathrm{~K}_{x} \mathrm{BiO}_{3}$ (for $x=$ $0.45)$ thin films for two different samples $i$ and $j$ prepared under slightly different annealing conditions(see Sato, et al. 48]).

Fig.I.4 Reflectivity spectra in the metallic regime $0 \leq x \leq 0.2$ of $\mathrm{BaPb}_{1-x} \mathrm{Bi}_{x} \mathrm{O}_{3}$ alloys. The solid curves are results of a Drude fit. Note the deviation of the Drude fit a $x=0.2$ (after Uchida et al. [2]).

Fig.I.5 Variation of the optical gap $E_{g}$ (i.e., the position of the peak in the optical absorption spectrum) and the transport gap $E_{A}$ (i.e., transport activation energy) in $\mathrm{eV}$, of $\mathrm{BaPb}_{1-x} \mathrm{Bi}_{x} \mathrm{O}_{3}$ with concentration $x$, in the semiconducting phase. The optical gap does not vanish at the semiconductor-metal transition. Two different scales have been used to show the wide separation of the energies of the two gaps (after Uchida, et al. [2]).

Fig.I.6a Optical conductivity spectrum (in a linear scale) in the semiconducting phase of $\mathrm{BaPb}_{1-x} \mathrm{Bi}_{x} \mathrm{O}_{3}$ alloys. Note the gradual shift of the conductivity peak towards high frequency with increasing $x$ (after Uchida, et al. [2]).

Fig.I.6b Optical conductivity of $\mathrm{Ba}_{1-x} \mathrm{~K}_{x} \mathrm{BiO}_{3}$ with $x=0.0$ (solid), $x=0.1$ (dot), $x=0.2$ (dash), $x=0.33$ (dot dash) and $x=0.4$ (solid) from Blanton, et al. [47].

Fig.I.7 (a) The composition dependence of the magnetic susceptibility at $150 \mathrm{~K}$ in $\mathrm{BaPb}_{1-x} \mathrm{Bi}_{x} \mathrm{O}_{3}$. The solid line is the diamagnetic contribution to susceptibility from the ionic core electrons (after Uchida, et al. [a]). In (b) is shown the normal state magnetic susceptibility of both lead and potassium doped systems as a function of temperature. The diamagnetic core contributions for the lead and potassium doped systems are, respectively, $8.6 \times 10^{-5} \mathrm{emu} / \mathrm{mole}$ and $7.8 \times 10^{-5} \mathrm{emu} / \mathrm{mole}$ (after Batlogg, et al. [24]). 
Fig.I.8a The temperature dependence of the resistivity of a single crystal thin-film sample of $\mathrm{BaPb}_{0.7} \mathrm{Bi}_{0.3} \mathrm{O}_{3}$ at different levels of oxygen deficiency. The labels 5 to 1 are in the increasing order of oxygen deficiency (after Enomoto et al. [63]).

Fig.I.8b The temperature dependence of the carrier concentration for a $\mathrm{BaPb}_{0.7} \mathrm{Bi}_{0.3} \mathrm{O}_{3}$ single-crystal thin filsm. The labels 5 to 1 are in order of increasing Oxyen deficiency (after Enomoto et al. [63]).

Fig.I.9 Temperature dependence of the upper critical field (in Tesla): (a) For $\mathrm{BaPb}_{1-x} \mathrm{Bi}_{x} \mathrm{O}_{3}$ at different filling $x . \mathrm{S}$ and $\mathrm{P}$ denote, respectively, single crystal and polycrystal samples. Doping $x$ and the superconducting $T_{c}$ of the samples are given with each curve (after Kitazawa, et al. [37]). (b) For $\mathrm{Ba}_{1-x} \mathrm{~K}_{x} \mathrm{BiO}_{3}$ thin films at $x=0.45$ (after Sato, et al. 48).

Fig.I.10 A spherically symmetric bismuth $6 s$ orbital surrounded by the oxygen p orbitals in the $\mathrm{BiO}_{6}$ octahedron. Only the $\sigma$ orbitals of each of the six oxygen atoms are shown.

Fig.I.11 LAPW band-structure results for $\mathrm{BaPb}_{1-x} \mathrm{Bi}_{x} \mathrm{O}_{3}$ alloys. The effect of $\mathrm{Pb}$ doping is shown from (c) through (a); $\sigma$ and $\sigma^{*}$ denote the bonding and antibonding $\sigma$ bands respectively. The antibonding band that crosses the Fermi level $E_{F}$ comes from the $\mathrm{Bi} / \mathrm{Pb} 6 \mathrm{~s}-\mathrm{O} 2{ }_{p} \sigma$ bonding. Lead doping adjusts the filling of the $\sigma^{*}$ subband and also introduces a noticeable chemical shift in the position of the $6 s$ band (open circles) (after Mattheiss [45]).

Fig.I.12 Comparison of schematic bands for the high- and low- $T_{c}$ oxide superconductors. (a) The Fermi level lies in the antobonding band of predominantly p character for the high- $T_{c}$ materials. (b) For the low $T_{c}$ materials the Fermi level is in the degenerate $\pi^{*}$ manifold. The quantities $\epsilon_{p}, \epsilon_{d}$, and $\epsilon$, are the energies of the ligand p-level and cation d and s levels, respectively (after Mattheiss [45]). 
Fig.I.13 Band-structure results for the lead-doped system with a tight-binding fit. (a) LAPW energy bands (solid lines) - for cubic $\mathrm{BaPb}_{0.7} \mathrm{Bi}_{0.3} \mathrm{O}_{3}$ compared with those derived from simple tight-binding models involving three (dotted lines) and five (dashed lines) tight-binding parameters respectively [44. Note the ten bands are the high-lying Ba-derived bands. (b) Sketch of the electron-like F-centered simple cubic Fermi surface predicted by the three-parameter tight-binding model for $\mathrm{BaBiO}_{3}$. Note that the electron surface nests with an identical hole surface centered at the Brillonin-zone corner (R) (after Mattheiss and Hamman [3]).

Fig.I.14 Model tight-binding conduction bands for cubic (dashed line) and distorted (solid line) $\mathrm{BaPb}_{1-x} \mathrm{Bi}_{x} \mathrm{O}_{3}$ alloys. The downward movement of the Fermi level as a function of doping is also shown. Note that at half filling the gap is at the Fermi surface (after Mattheiss 45]).

Fig.I.15 (a) LAPW band-structure results for the $\mathrm{Ba}_{1-x} \mathrm{~K}_{x} \mathrm{BiO}_{3}$ system. The wide bands of Bi6s-O2p are bounded above and below by the $\sigma^{*}$ and $\sigma$ bands. The Fermi level lies in the $\sigma^{*}$ band. Note the similarity between the band structures of the K-doped and Pb-doped systems (after Mattheiss and Hamman [3, 45]). (b) The full potential LMTO band structure near fermi level for $2 \mathrm{BaBiO}_{3}$. Solid line is for $t=0^{\circ}$ and $b=0 \AA$, dashed line is for $t=10^{\circ}$ and $b=0 \AA$, dot-dashed line is for $t=10^{\circ}$ and $b=0.09 \AA$. The brillouin zone has been folded (after Liechtenstein, et al. 46]).

Fig.I.16 $T_{c}$ versus the Sommerfeld constant $\gamma$ for various superconductors. bismuthates and cuprates have the highest $T_{c}$ 's on an absolute scale (and especially given their low DOS at $E_{F}$ ). The extraordinary character of these two types of systems relative to all other superconductors, including oxides, is highlighted in the above plot. The dashed line demarcates low- $T_{c}$ superconductors from the high- $T_{c}$ superconductors (after Batlogg [7]). 
Fig.II.1 $T_{c}$ as a function of $U_{\text {eff }} / 2 \mathrm{~V}$ calculated in the Hartree approximation for the BEG model (lower curve). Solid lines denote second order and dashed lines denote first-order transitions and the dot, a tricritical point. In the Hartree calculations $U_{o}$ and $e^{2} / \alpha a$ were kept fixed at the values 2 and 1 , respectively, and $g^{2} / \mathrm{C}$ is varied (after Rice and Sneddon [78).

Fig.II.2 $T_{c}$ as a function of $\bar{z}$, the average coordination number in the $\mathrm{BaPb}_{1-x} \mathrm{Bi}_{x} \mathrm{O}_{3}$ system for three different values of $U_{e f f} / 2 \mathrm{~V} . T_{c}$ is calculated in the Bethe-Peierls approximation. The inset on the top right corner shows the effect of a nonzero band with $\left(t_{i j} \neq 0\right)$ in this phase diagram, on the region near zero temperature, as described in the text, for $U_{\text {eff }} / 2 V>0$. In this diagram $\mathrm{M}$ is a metallic phase and S stands for BCS superconductor (after Sneddon and Rice [78).

Fig.II.3a The DOS of the Bi A-site (solid line) and Bi B-site (dashed line) at 20\% $\mathrm{Pb}$ doping using $\epsilon_{o}=2 \mathrm{eV}$ and $\lambda=0.8$. The Bi subbands approach each other because of a level repulsion between the $\mathrm{Bi}$ and $\mathrm{Pb}$ single bands. The total DOS is shown in the inset at the top right corner. The different level shifts of the Bi subbands produce a pseudogap around the Fermi energy. In (b) is shown the case of $60 \% \mathrm{~Pb}$ doping using the same parameter values as in (a). The Bi subbands continuously approach each other with increasing $\mathrm{Pb}$ doping. The states around the Fermi energy are still almost composed of Bi sites (after Jurczek and Rice [79]).

Fig.II.4a The boundary between semiconducting and metallic phases in the $\epsilon^{(0)}-x$ plane for several choices of $U$. The symbols are explained in the text. In (b) is shown the DOS obtained in the CPA at two different values of $U$ (after Yoshioka and Fukuyama 89]).

Fig.II.5 The superconducting transition temperature as a function of filling $x$ for different values of $U$ with $\epsilon^{(0)}$ kept fixed. Notte that the phase diagram is symmetric with 
respect to $x \longrightarrow 1-x$ (after Yoshioka and Fukuyama [89]).

Fig.III.1a A typical phase diagram of the pseudospin model in the pseudomagnetic field $(B)$ and temperature $(T)$ plane. The full lines are second-order phase boundaries and the dashed lines indicate first-order phase boundaries. The two continuous lines and the first-order line meet at a bicritical point (the full circle). The first-order line separates the charge-density-wave (CDW) phase (antiferromagnet in the spin language) from the singlet superconducting (SS) (spin flopped in the spin language) phase. The two continuous lines separate the non-ordered metallic (NOM) phase (paramagnet in the spin language, with a net moment because of the aligning field) from the CDW and SS phases. The arrows show the arrangement of the spins on nearest-neighbour sites. The parameters used are $J_{o}=1.0$ and $K_{o}=1.301$.

Fig.III.1b The phase diagram of Fig.III.1a in the magnetisation $(m)$ and temperature (T) plane. The full lines are second-order phase boundaries and the dashed lines indicate first-order phase boundaries. The hatched region is the two-phase coexistence region (SS and CDW) corresponding to the first-order phase boundary between CDW and SS phases in Fig.III.1a.

Fig.III.2 The mean-field phase diagram when a next nearest neighbour interaction is included in model II.5 (in the large- $U$ limit). The phase denoted by I is the so called intermediate phase (after K.-S. Liu and M.E. Fisher, J. Low Temp. Phys. 10, 655-683 (1973)).

Fig.III.3 The RPA excitation spectra of the pseudospin model shown along different symmetry directions of the Brillouin zone of a cubic lattice (see inset), with $J_{o}=$ 1.0 and $K_{o}=1.301$. The lines marked $\mathrm{CDW}(+)$ and $\mathrm{CDW}(-)$ correspond to the upper and lower branches of the spectrum in the CDW phase (for temperature $T=0.25$, pseudomagnetic field $B=0.25$, magnetisation $m=0.4$ and staggered 
magnetisation $\eta=0.96)$. The dashed line shows the spectrum for the SS phase (at $T=0.25, B=0.70, m=0.61$ and $\theta_{A}=\theta_{B}=50.55^{\circ}$, where $\theta_{A} \& \theta_{B}$ are defined in the text). The dash-dotted line shows the spectrum in the NOM phase (at $T=0.80, B=0.40$ and $m=0.27$ ). The spectrum in the $\mathrm{SS}$ phase is gapless whereas the spectrum in the CDW phase has the anisotropy gap discussed in the text.

Fig.III.4 The optical conductivity (in arbitrary units) in the large- $U$ limit using RPA plotted as a function of frequency. The parameter values taken are $z t=1 \mathrm{eV}, U=2$ $\mathrm{eV}$, and $z V=0.05 \mathrm{eV}$. The same phenomenological broadening $(\Gamma=0.09)$ has been used for both the channels. Note that the two-particle absorption peak is a factor of about 9 down compared to the one-particle peak.

Fig.IV.1(a) The mean-field phase diagrams of the negative-U, extended Hubbard model in filling-temperature plane, for two different values of the interaction parameters. The solid lines and the hatched region have the same meaning as in Fig.III.1. The values of Coulomb interactions and zt used to obtain this phase diagram are shown in the figure.

Fig.IV.1b The mean-field phase diagram of the negative-U, extended Hubbard model in the chemical potential-temperature plane. The solid and the dashed lines denote continuous and first-order transition, respectively as in Fig.III.1a. Other symbols also have the same meanings as in Fig.III.1.

Fig.IV.2 (a) The single-particle excitation spectrum in the mean-field CDW phase where $\Delta_{c}$ is the CDW gap parameter. In (b) are shown, schematically, the two-particle (hole) bound states that form inside the gap of the two-particle spectrum. The energy of these bound states is the transport gap $\left(E_{A}\right)$ in our theory.

Fig.IV.3 The diagrammatic representation of the RPA series for the pair susceptibility 
in the mean-field CDW state. Double lines denote mean-field single-particle Green

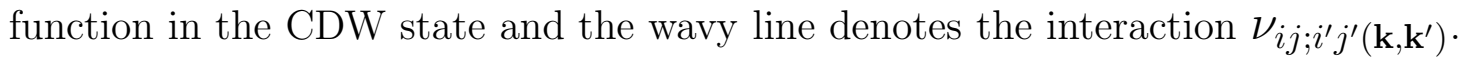

Fig.IV.4 A schematic representation of the Cooper instability equation as described in the text.

Fig.IV.5a The bare (empty circles) and the screened (empty triangles) onsite Coulomb interactions as functions of (half) the band width. The screening mechanism is explained in the text (see also Fig.IV.7).

Fig.IV.5b The extended Coulomb term $V(\times 2 z)$ as a function of (half) the band width $W(=z t)$. The condition that we have used to obtain this graph is that the bound state energy should be equal to the transport gap (see the text). Note that in the bandwidth $\rightarrow 0$ limit the value of $2 z V$ approaches $0.24 \mathrm{eV}$, the result obtainted in the large- $U$ limit.

Fig.IV.6 The Coulomb interactions $U$ and $z V$ as a functions of the bound-state energy. This graph is obtained with the condition that the CDW gap parameter is half the optical gap of $\mathrm{BaBiO}_{3}$ (i.e., the CDW gap is $1 \mathrm{eV}$ ). Note that $V$ goes to zero as the bound-state energy approaches zero.

Fig.IV.7(a) A diagrammatic representation of the screening (in RPA) of the particleparticle interaction. Note that the particle-particle interaction is screened by the particle-hole excitations.

Fig.IV.7b Same as (a) for the particle-hole interaction. The particle-hole interaction is screened by the particle-particle processes. Note that our calculation is not self consistent (see text).

Fig.IV.8 (a) The superconducting transition temperature with and without the screening described in the text. (b) The CDW transition temeperature with and without the 
screening.

Fig.IV.9 The superconductor-nonordered and the CDW -nonordered phase boundary evaluated with the same parameters as in IV.1b. To show that screening of $V$ does not much affect the superconductor-nonordered phase boundary at the range of parameter values we are interested in, we draw this phase boundary for $V=0$ also (dashed line). Note that this boundary is quite close to the boundary with $2 z V=0.13 \mathrm{eV}$ (the solid and the dashed line both represent continuous phase transitions.) 